\title{
Functional Characterization of Hereditary Spastic Paraplegia Proteins Spastin and ZFYVE27
}

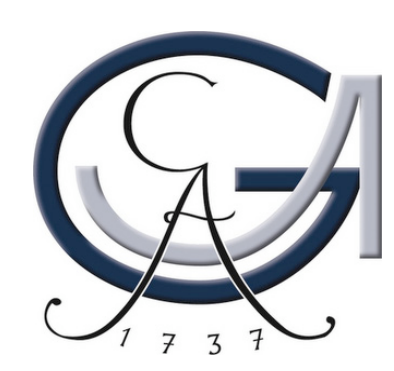

\section{Dissertation}

zur Erlangung des Doktorgrades

der Mathematisch-Naturwissenschaftlichen Fakultäten der Georg-August-Universität zu Göttingen

\section{von}

D. V. Krishna Pantakani

aus Tadimalla, Indien

Göttingen, 2009 
D7

Referent: Prof. Dr. Dr. Wolfgang Engel

Korreferent: Prof. Dr. Sigrid Hoyer-Fender

Tag der mündlichen Prüfung: 
Dedicated to my family,

whose love, faith in me, and wishes for my success has helped me in my path. 


\section{Table of Contents}

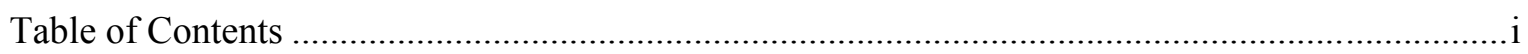

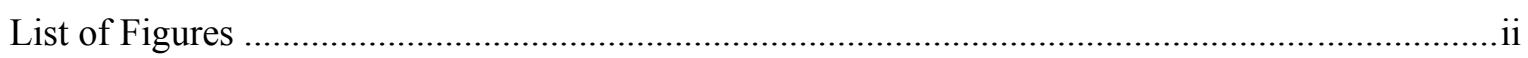

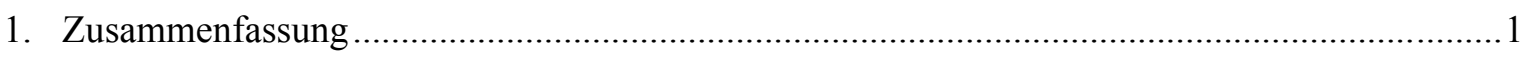

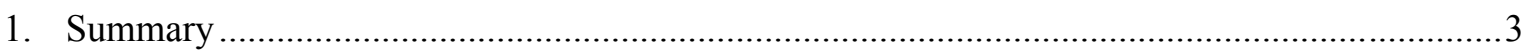

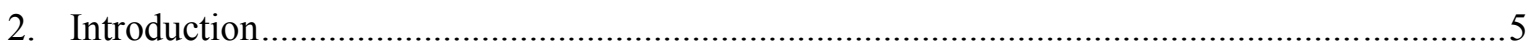

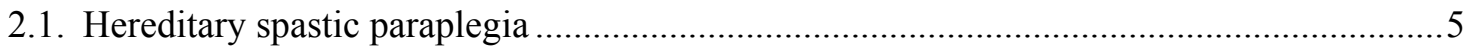

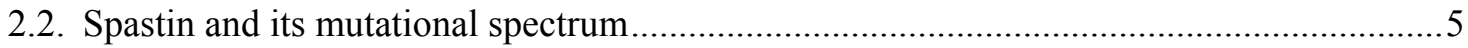

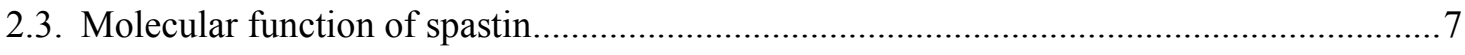

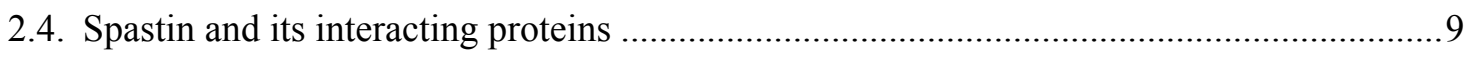

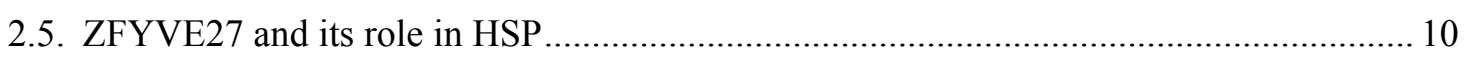

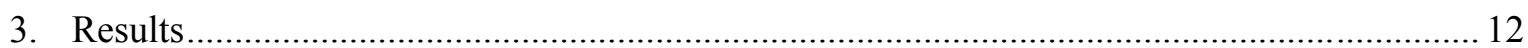

3.1. Spastin oligomeizes into a hexamer and the mutant spastin (E442Q) redistributes the

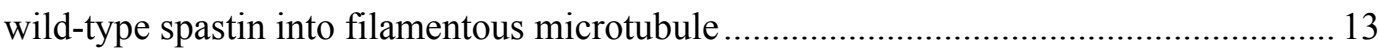

3.2. Compound heterozygosity in $S P G 4$ gene causes hereditary spastic paraplegia................ 26

3.3. Expansion of mutation spectrum, determination of mutation cluster regions and predictive structural classification of SPAST mutations in hereditary spastic

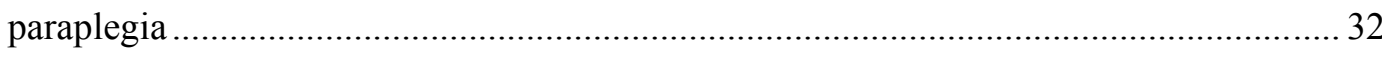

3.4. ZFYVE27 (Protrudin) oligomerize as dimer/tetramer and truncated ZFYVE27 $\left(\triangle \mathrm{HR} 3^{184-208}\right)$ deters the ability of wild type ZFYVE27 to promote neurite extension ..... 41

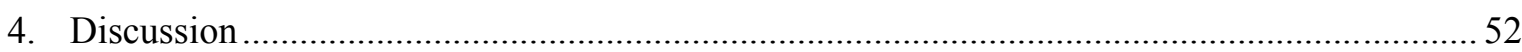

4.1. Spastin assembles into hexamer for its microtubule severing activity .............................52

4.2. Expansion of mutational spectrum and evaluating the effects of spastin mutations in

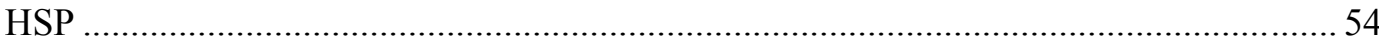

4.3. Elucidating the molecular function of ZFYVE27, a spastin interacting protein...............56

4.4. Generation of knock-out mouse model for Zfyve27 …..................................................... 58

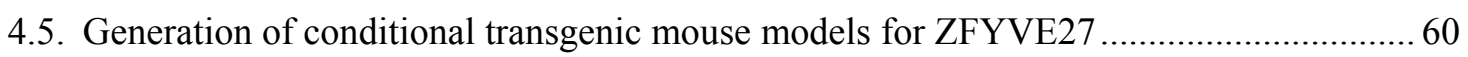

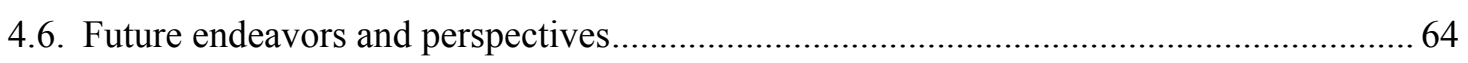

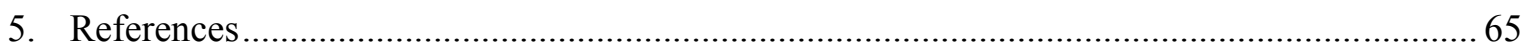

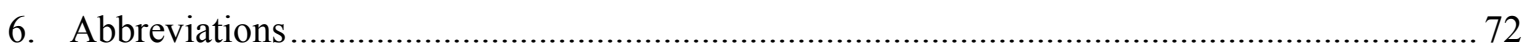

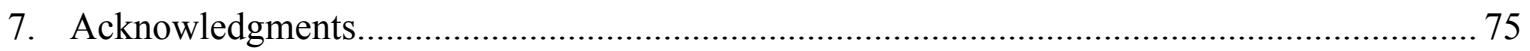

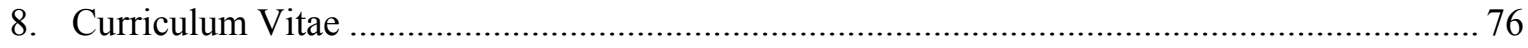

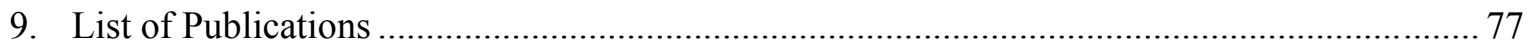

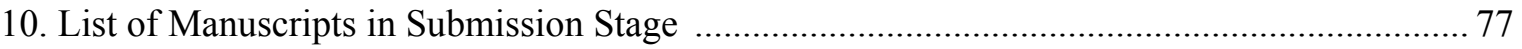




\section{List of Figures}

Figure 2.1. Schematic representation of a neuron indicating the localization of identified mutant HSP proteins .6

Figure 2.2. Structural architecture and different isoforms of spastin ..........................................

Figure 2.3. Schematic representation of axon undergoing the branch formation.............................. 9

Figure 4.1. Structural basis for the hexameric spastin pore loops formation and model for

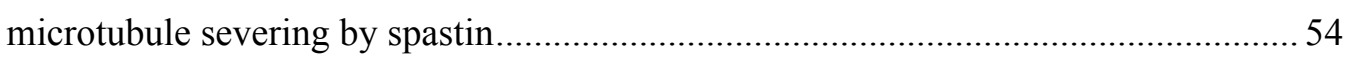

Figure 4.2. Model for multivalent membrane binding by FYVE domain of homodimeric EEA1 .. 57

Figure 4.3. Schematic diagram depicting the generation of Zfyve27 knock-out targeting construct.

Figure 4.4. Schematic representation of genomic locus for Zfyve27 wild type and knock-out alleles 60

Figure 4.5. Schematic illustration of conditional transgenic constructs 62

Figure 4.6. Schematic outline showing conditional activation of transgenes. 63 


\section{Zusammenfassung}

Die Hereditären Spastischen Paraplegien (HSPs) sind eine heterogene Gruppe von genetischen Erkrankungen, die durch progressive spastische Lähmungen in den unteren Gliedmaßen gekennzeichnet sind, was hauptsächlich durch Funktionsstörungen der Pyramidenbahn-Neuronen verursacht wird. Alle Arten der Vererbung, wie autosomal dominant (AD), autosomal rezessiv (AR) und X-chromosomal, sind für HSP bekannt. Mutationen im SPG4/SPAST Gen sind der häufigste Grund für AD-HSP und machen bis $\mathrm{zu} 40 \%$ aller AD-HSP Fälle aus. In der vorliegenden Arbeit haben wir die funktionellen und strukturellen Eigenschaften des SPAST Genprodukts Spastin und dessen interagierenden Proteins ZFYVE27, ein HSP zugehöriges Protein, untersucht.

Im ersten Teil der Arbeit wurde der oligomere Status von Spastin mit Hilfe von biochemischen und zellulären Ansätzen als Hexamer identifiziert. Außerdem wurden durch die Modellierung der Spastin AAA Domäne strukturelle Informationen über das SpastinHexamer erhalten. Diese modellierte Struktur diente wiederum als Gerüst, um die bekannten Spastin Mutationen bei HSP Patienten in vier funktionelle Gruppen einzuteilen.

Im zweiten Teil der Arbeit wird über compound heterozygote Sequenzvarianten von SPAST berichtet, die ein frühes Auftreten des HSP Phänotyps verursachen. Ein "'modifier effect' des bekannten Spastin-Polymorphismus (S44L) in Verbindung mit einer anderen Mutation wird beschrieben. Unsere Ergebnisse festigen ein Schwellenwert Modell von Spastin mit einer direkten Korrelation zwischen funktionellem Spastin-Level und frühem Auftreten des HSP Phänotyps.

Im dritten Teil konnte eine Erweiterung des Spastin-Mutationsspektrums in einer großen HSP Kohorte mit 29 neuen Spastin-Mutationen beschrieben werden. Außerdem wurde versucht, alle möglichen negativen Effekte dieser neuen Mutationen auf die oligomere Funktion von Spastin mit Hilfe des Spastin Strukturmodells zu ermitteln.

Im letzten Teil dieser Arbeit konnte mit Hilfe von zellulären und biochemischen Analysen gezeigt werden, dass ZFYVE27, ein mit Spastin interagierendes Protein, als Dimer/Tetramer wirkt. Weiterhin haben wir gezeigt, dass die Hauptinteraktionsregion, die hydrophobe Region 3 (HR3), zwar nicht wichtig für die Selbstassoziation von ZFYVE27 ist dafür aber essentiell für die Neuritenbildung. Die Coexpression des verkürzten ZFYVE27 $\left(\Delta \mathrm{HR} 3^{184-208}\right)$ erschwert die Funktion des Wild-Typ ZFYVE27. 
Außerdem wurde damit begonnen, "loss of function" und "gain of function" Mausmodelle für ZFYVE27 herzustellen. Damit kann die Rolle von ZFYVE27 im HSP Pathomechanismus studiert wurden. Denkbarerweise könnte das "loss of function" Mausmodell die phänotypischen Merkmale von HSP nachahmen. Mit der Analyse der Rolle von ZFYVE27 in der Neuritogenese/Neuropathie in den Mausmodellen wird es möglich sein, neue Erkenntnisse über den Pathomechanismus von HSP zu gewinnen. 


\section{Summary}

Hereditary spastic paraplegias (HSP) are a heterogeneous group of genetic disorders characterized by progressive spasticity in the lower limbs caused primarily due to dysfunction of corticospinal tract neurons. All modes of inheritance such as autosomal dominant (AD), autosomal recessive (AR) and X-linked have been reported for HSP. Mutations in the SPG4/SPAST gene are the single most common cause for AD-HSP and accounts for up to $40 \%$ of all AD-HSP cases. In the present study, we have characterized the functional and structural properties of SPAST gene product, spastin and its interacting protein ZFYVE27, another HSP related protein.

In the first part of this thesis, spastin oligomeric state as hexamer has been evaluated by biochemical and cellular approaches. Furthermore, structural information on hexameric spastin was obtained through modeling of spastin AAA domain and this modeled structure was used as a framework to classify the known mutations of spastin in HSP patients into four different functional groups based on the structural information.

In the second part, compound heterozygous sequence variants of SPAST causing early age onset of HSP phenotype has been reported. The "modifier effect" of spastin known polymorphism (S44L) in association with another mutation has been described. Our findings further strengthen the 'threshold effect model of spastin, with a direct correlation between functional level of spastin and early age onset of HSP phenotype.

In the third part, expansion of spastin mutational spectrum in a large HSP cohort has been reported with identification of 29 novel spastin mutations. Moreover, we attempted to determine any possible deleterious effects of these novel mutations on spastin oligomeric function using spastin structural model.

In the last part of this thesis, we showed that ZFYVE27, a spastin interacting protein, functions as dimer/tetramer by means of cellular and biochemical analysis. Further, we show that although the core interaction region, hydrophobic region 3 (HR3) is dispensable for self-association of ZFYVE27, it is essential for neurite formation and co-expression of the truncated ZFYVE27 ( $\triangle \mathrm{HR} 3^{184-208}$ ) hampers the function of wild type ZFYVE27.

Moreover, to dissect the role of ZFYVE27 in HSP pathomechanism, we endeavored to generate "loss of function" as well as "gain of function" mouse models for ZFYVE27. Conceivably, the phenotype of "loss of function" mouse model might mimic the clinical features of HSP. By elucidating the role of ZFYVE27 in neuritogenesis/neuropathies by 
means of generated mouse models, we will be able to gain novel mechanistic insights into the underlying pathomechanism of HSP. 


\section{Introduction}

\subsection{Hereditary spastic paraplegia}

Hereditary spastic paraplegias (HSPs), also known as Strümpell-Lorrain familial spasmodic paraplegias, are a group of neurological disorders that are characterized by progressive spasticity and weakness predominantly in the lower limbs (Harding, 1983). The common pathological cause for this disease is distal axonal degeneration of the longest nerve fibers in the corticospinal tracts and posterior columns (Behan and Maia, 1974; Deluca et al., 2004). In clinical terms, HSPs can be classified as 'pure' or 'complex' depending on whether or not the spastic paraplegia is associated with additional neurological and systemic abnormalities.

HSPs are genetically heterogeneous and all modes of inheritance have been reported, such as autosomal dominant (AD), autosomal recessive (AR) and X-linked. The mapping of 37 different loci (17 of which have been identified) further highlights the genetic heterogeneity of the disease. Moreover, the studies on identified HSP genes revealed their role in axonal transport and vesicle trafficking thus suggesting that impairment of such processes are the underlying cause for HSP as illustrated in figure 2.1. The AD-HSPs are almost 'pure' in terms of clinical features with weakness and spasticity in the lower limbs (Harding, 1981; Durr et al., 1994; Fink, 2003), whereas AR forms of HSP appear to be 'complex' and comprise a large number of conditions in which spastic paraplegia is associated with other neurological features such as ataxia, severe amyotrophy, optic atrophy, mental retardation, dementia, deafness, ichthyosis, peripheral neuropathy and epilepsy (Harding, 1993; Fink, 2003). The most common form of AD-HSP is caused by mutations in SPG4/SPAST gene, which accounts for approximately $40 \%$ of the AD-HSP cases.

\subsection{Spastin and its mutational spectrum in HSP}

The SPAST gene encodes for a 616 amino acid protein named spastin. Spastin belongs to AAA (ATPase Associated with various cellular Activities) family of proteins and it was shown to be a microtubule (MT) severing enzyme. Spastin has different isoforms depending on the usage of an alternative translation initiation codon and on alternative splicing of the exons, in particular exon 4 (Fig. 2.2). Spastin's protein 


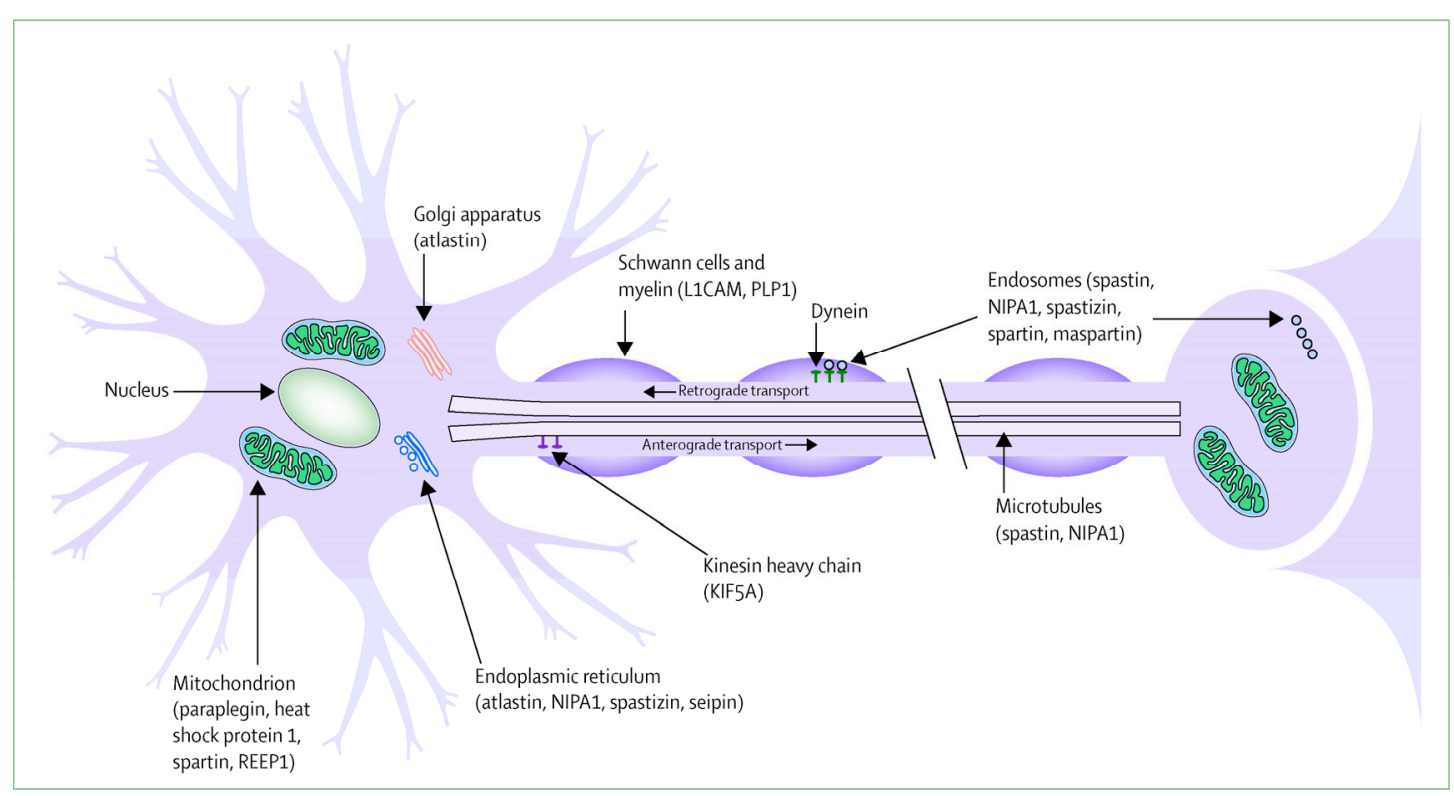

Figure 2.1. Schematic representation of a neuron indicating the localization of identified mutant HSP proteins (in parenthesis - gene name or locus name); Mitochondria associated - paraplegin (SPG7), heat shock protein 1 (SPG13), spartin (SPG20) and REEP1 (SPG31); Endoplasmic reticulum associated - atlastin (SPG3A), NIPA1 (SPG6), spastizin (SPG15) and seipin (SPG17); Golgi associated - atlastin (SPG3A); molecular motors associated - kinesin heavy chain (SPG10); microtubule associated - spastin (SPG4) and NIPA1 (SPG6); Endosomes associated - spastin (SPG4) and NIPA1 (SPG6), spastizin (SPG15), spartin (SPG20), maspardin (SPG21); Myelin sheath and Schwann cells associated - L1CAM (SPG1), PLP1 (SPG2). This wide cellular distribution, but closely related functions of identified HSP proteins suggest that impaired transport of macromolecules and organelles, disturbances of mitochondrial function are the primary cause of HSP pathomechanism (adapted from Salinas et al., 2008).

structure is defined by three major functional domains, namely; MIT-microtubule interacting and trafficking domain, MTBD-microtubule binding domain and a catalytic AAA-ATPase domain (Fig. 2.2). Apart from these three functional domains, additional motifs like nuclear localization signals (NLS), nuclear export signals (NES) (Beetz et al., 2004; Claudiani et al., 2005) and also a putative transmembrane domain has been identified in the primary sequence of spastin. More than 200 different mutations/DNA alterations have been reported in SPAST gene including missense, nonsense, splice site mutations, small insertions and small deletions, which are summarized in the Human Gene Mutation Database (http://portal.biobaseinternational.com/hgmd/pro/start.php). Moreover, recent studies reported gene rearrangements, in particular large genomic deletions in $S P A S T$ by using SPAST specific Multiplex Ligation-dependent Probe 
Amplification (MLPA), which account for approximately 18-20\% of HSP cases (Beetz et al., 2006; Depienne et al., 2007). This broad mutational spectrum of SPAST-HSP suggests haploinsufficiency as the likely pathomechanism.

From a diagnostic point of view, unfortunately, there are no common mutations in $S P A S T$, with most families having private mutations. Interestingly from several recent clinical and molecular studies, it is emerging that besides haploinsufficiency, other modes of action such as "dominant-negative" and "threshold-effect-model" are relevant for at least a sub-set of spastin mutations (Errico et al., 2002; Chinnery et al., 2004; Svenson et al., 2004). However, till now only a single loss-of-function mouse model for spastin was generated and these mice manifest only a mild and late-onset of some of the HSP-likephenotype (Tarrade et al., 2006). To elucidate a comprehensive function of spastin and to test different modes of disease mechanism, generation/characterization of additional mouse models including missense mutation(s) are necessary.

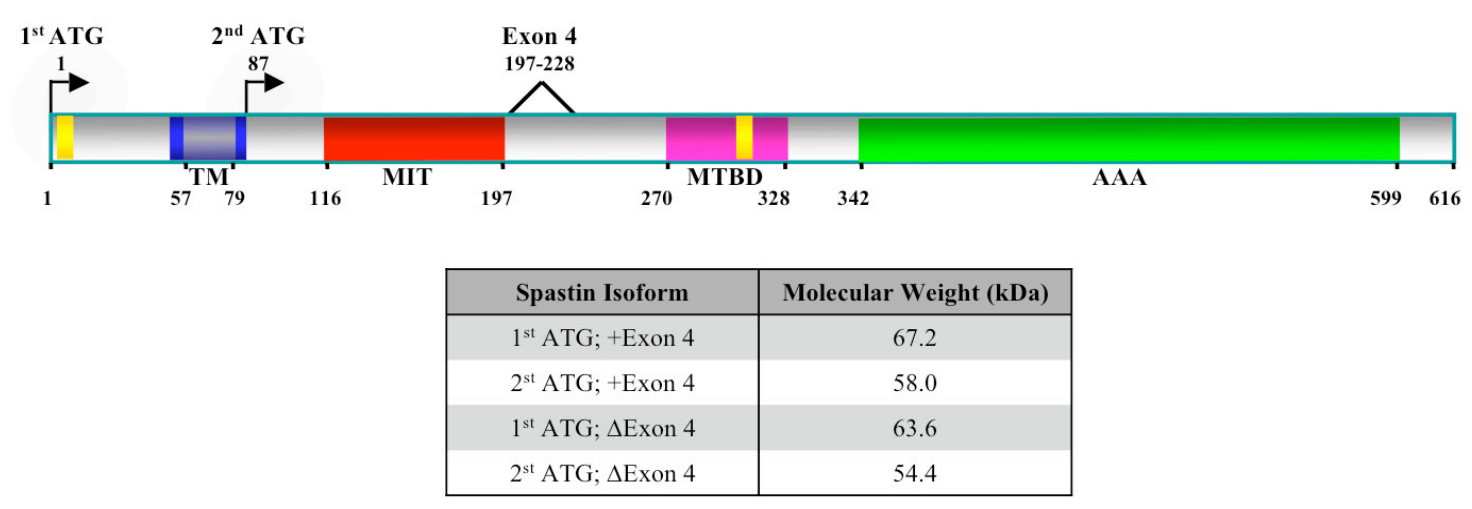

Figure 2.2. Structural architecture and different isoforms of spastin. The spastin protein contains three main domains; MIT-microtubule interacting and trafficking, MTBD-microtubule binding domain and AAAATPase domain. Additionally, spastin contains two NLS-nuclear localization signals (aa 4-11 and 309-312), which are highlighted in yellow, one NES-nuclear export signal (aa 50-87) highlighted in blue and a TMtransmembrane domain highlighted in grey. Different isoforms of spastin, which are reported depending on the usage of first or second ATG and the presence or absence of exon 4 are summarized with predicted molecular weights of the protein isoforms in the table (adapted from Salinas et al., 2005).

\subsection{Molecular function of spastin}

Initial studies suggested that spastin is ubiquitously expressed in all tissues (Hazan et al., 1999). To date, the majority of the studies reported only a cytoplasmic function of 
spastin such as a role in MT dynamics and in cytokinesis. During cell division, spastin could be detected in the spindle pole, the central spindle, and in the midbody (Errico et al., 2004). In motor neurons, spastin is enriched in regions where dynamic MTs regulation takes place (Errico et al., 2004), it also localizes in axon and dendrites (Trotta et al., 2004). Spastin's central role in MT dynamics is evident from the observation of diminished MT network in cells overexpressing wild-type spastin (Errico et al., 2002) and from the high sequence homology with the well characterized MT severing enzyme P60-katanin (Beyer, 1997; Errico et al., 2002).

In an attempt to elucidate the molecular function of spastin in MT dynamics, numerous studies have used various biological systems. Downregulation of spastin in Drosophila by RNA interference (RNAi) caused the morphologic undergrowth, reduced synaptic area and increased synaptic MT (Trotta et al., 2004). Further, several lines of experimental evidences using human and Drosophila orthologues of spastin have demonstrated that spastin indeed functions as MT severing enzyme (Errico et al., 2002; Trotta et al., 2004; Evans et al., 2005; Salinas et al., 2005; Roll-Mecak and Vale, 2005). Expression of spastin ATPase activity deficient mutants, including pathogenic spastin HSP mutations, did not severe MT, indicating that ATPase activity of spastin is crucial for MT severing (Evans et al., 2005; Roll-Mecak and Vale, 2005). On the other hand, spastin and some of its ATPase mutants showed MT bundling as assessed by staining of detyrosinated Glu-tubulin (Evans et al., 2005) and was found to be independent of ATP hydrolysis (Salinas et al., 2005). Hence, it is likely that spastin may have both MT severing and bundling activity.

Strikingly, intracellular distribution analysis in neuronal cells revealed that spastin is concentrated at the axonal branch sites near the junction-point of neurite formation, but P60-katanin is distributed ubiquitously in axons and neurites ( $\mathrm{Yu}$ et al., 2008). Overexpression of spastin in rat hippocampal neurons caused an increase in axonal branching, from which new neurite extensions are generated (Yu et al., 2008). In contrast, overexpression of P60-katanin had no significant effect on the number of branch sites and filopodia formation ( $\mathrm{Yu}$ et al., 2008). Interestingly, spastin and P60-katanin show a variable level of sensitivity to protection of MT by MT stabilizing proteins such as microtubule associated protein tau (MAPT/tau) or microtubule associated protein 4 (MAP4) (Yu et al., 2008). Taken together, these studies suggest that the local detachment 
of MAPT/tau by phosphorylation enhances the MT severing activity of katanin at sites of branch formation (Fig. 2.3A), whereas spastin MT severing activity at site of branch formation is enhanced by the enrichment of spastin molecules at local sites and is independent of tau MT protection (Fig. 2.3B). These two modes are not mutually exclusive and may work in cooperation (Yu et al., 2008).

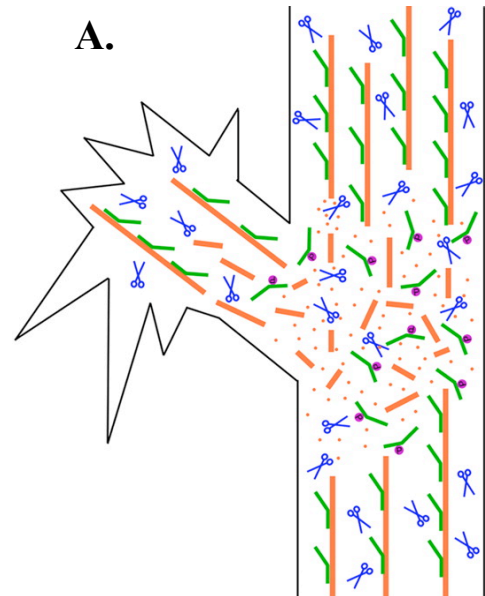

Katanin Mode

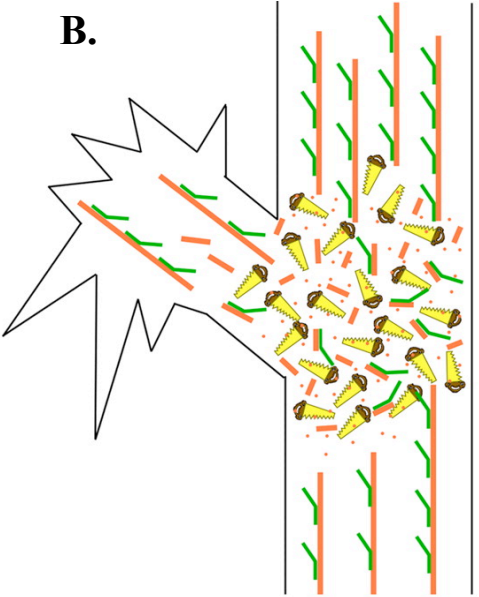

Spastin Mode

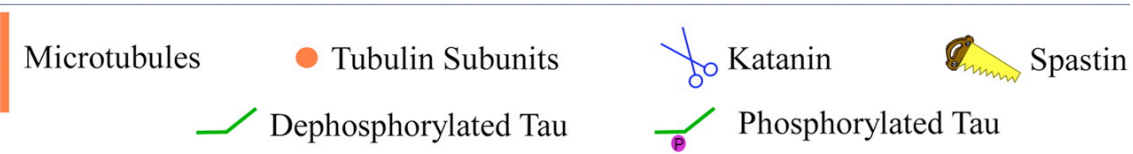

Figure 2.3. Schematic representation of axon undergoing the branch formation. (A) In the 'katanin mode' local detachment of the microtubule protecting protein tau enables the microtubule severing activity of katanin at sites of branch formation. (B) In the 'spastin mode', microtubule severing is enhanced by the local enrichment of spastin and is independent of tau protection (adapted from Yu et al., 2008).

\subsection{Spastin and its interacting proteins}

Significant insights into molecular function of spastin were derived from the identification and characterization of spastin binding proteins. Till to date, several spastin interacting proteins were identified, namely; reticulon1 (RTN1), atlastin, CHMP1B, ZFYVE27 (protrudin) and NA14 (Errico et al., 2004; Evans et al., 2006; Mannan et al., 2006a, b; Reid et al., 2005). Interestingly, all the known spastin binding proteins are components of vesicular transport processes except for NA14. RTN1 and atlastin are the components of endoplasmic reticulum (ER) derived secretory pathway (Evans et al., 2006; Mannan et al., 2006a), which is equivalent to axoplasmic anterograde transport in neurons. RTN1 belongs to the reticulon (RTN) family of proteins, which are primarily localized in 
the ER (Oertle and Schwab, 2003; van de Velde et al., 1994). RTN family members are characterized by a highly conserved reticulon homology domain, which consists of about 188 amino acids with two putative transmembrane regions separated by a 66-residue loop (Oertle and Schwab, 2003). RTN1 is known to be involved in intracellular transport events including regulated exocytosis and it is expressed in the nervous system, particularly in those tissues that are susceptible to neurodegeneration (Steiner et al., 2004).

Atlastin is encoded by $S P G 3 A$ gene; it belongs to the dynamin/guanylate-binding protein superfamily of large GTPases (Guanosine triphosphatases). Mutations in the $S P G 3 A$ gene are the second most common cause for HSP and most of the identified disease mutations are missense mutations that affect the GTPase function of the protein (Zhu et al., 2006; Namekawa et al., 2007). Atlastin oligomerizes as tetramers and it is likely that most of the identified missense mutations may exert a dominant negative effect on the tetrameric atlastin ( $\mathrm{Zhu}$ et al., 2003). Intracellular distribution studies with the endogenous protein show a predominant localization in the cis-Golgi, with additional localizations in the ER and in punctate cytoplasmic structures (Zhu et al., 2006; Namekawa et al., 2007). In the developing primary cortical neurons, atlastin was enriched in vesicular structures in axonal growth cones and branch sites thus suggesting its role in axonal development (Zhu et al., 2006).

Interestingly, CHMP1B (CHarged Multivesicular body Protein 1B/Chromatin modifying protein 1B), and ZFYVE27 are suggested to be involved in endosomal membrane traffic at multiple levels. They are the components of endocytic pathway or retrograde axonal transport system in the neurons (Reid et al., 2005; Mannan et al., 2006b). CHMP1B is an endosomal protein and it is closely associated with the endosomal sorting complex required for transport-III (ESCRT-III) complex (Reid et al., 2005). Four distinct ESCRTs, known as ESCRT- 0, -I, -II and -III, facilitate the trafficking of ubiqutylated proteins from endosomes to lysosomes for degradation via multivesicular body (MVB) formation (reviewed by Williams and Urbe, 2007).

\subsection{ZFYVE27 and its role in HSP}

ZFYVE27 is a member of FYVE (Fab1p, YOTB, Vac1p and EEA1) family of proteins and is enriched in the endosomal membranes of the cell (Mannan et al., 2006b). The FYVE domain is characterized by a zinc binding finger motif and is involved in 
membrane trafficking (Stenmark et al., 1996). This conserved FYVE domain ( 70-residue module) specifically recognizes phosphatidyl inositol 3-phosphate (PtdIns3P) and targets many cytosolic proteins to PtdIns3P enriched endosomal membranes (Burd and Emr, 1998; Gaullier et al., 1998; Patki et al., 1998). The FYVE finger proteins have different biological functions ranging from endocytotic transport, regulation of endosomal membrane fusion with other endocytic vesicles/organelles (Peterson et al., 1999; Nielsen et al., 2000; Rubino et al., 2000; Morino et al., 2004) and in signal transduction (Tsukazaki et al., 1998; Sasaki and Sugamura, 2001).

ZFYVE27 was identified as a spastin interacting protein and more importantly, a missense mutation (p.G191V) in ZFYVE27 was reported in a five generation German family with HSP (Mannan et al., 2006b). The role of ZFYVE27 and spastin in a molecular process relevant to HSP is highlighted by recent functional studies on ZFYVE27 and spastin in neurons (Shirane and Nakayama, 2006; Yu et al., 2008). ZFYVE27/Protrudin plays vital function in neuronal differentiation and along with Rab11 plays a central role in directional membrane trafficking and neurite formation (Shirane and Nakayama, 2006). Interestingly, overexpression of spastin in primary hippocampal neurons lead to extensive neurite outgrowth (axonal branch site formation) (Yu et al., 2008) similar to that observed for ZFYVE27. Spastin, ZFYVE27 and Rab11 may together constitute a system for the regulation of vesicular transport in neurons and impairment of this system may be responsible for the pathogenesis of AD-HSP.

To gain further insights into spastin and ZFYVE27 molecular function and their physiological interaction in a common cellular pathway relevant to HSP pathogenesis, the current study is aimed at understanding the molecular structure of spastin, evaluating the effects of spastin mutations in HSP patients and characterization of ZFYVE27 molecular function. Further, generation of 'loss of function' mouse model and 'gain of function' mouse models for ZFYVE27 were undertaken to elucidate its role in HSP. 


\section{Results}

3.1. Spastin oligomerizes into a hexamer and the mutant spastin (E442Q) redistribute the wild-type spastin into filamentous microtubule.

3.2. Compound heterozygosity in SPG4 gene causes hereditary spastic paraplegia.

3.3. Expansion of mutation spectrum, determination of mutation cluster regions and predictive structural classification of SPAST mutations in hereditary spastic paraplegia.

3.4. ZFYVE27 (Protrudin) oligomerize as dimer/tetramer and truncated ZFYVE27 $\left(\triangle \mathrm{HR} 3^{184-208}\right)$ deters the ability of wild type ZFYVE27 to promote neurite extension.

Each chapter within the results starts with a brief description of the aim of the particular manuscript in context of the complete thesis, the status of the manuscript, and the author's contribution to the work. 


\subsection{Spastin oligomerizes into a hexamer and the mutant spastin (E442Q) redistribute the wild-type spastin into filamentous microtubule.}

In the first part of the thesis, oligomerization of spastin (encoded by $S P G 4 / S P A S T$ ), the most commonly mutated gene in hereditary spastic paraplegia (HSP) was reported. By means of chemical crosslinking and size exclusion chromatography, spastin was shown to oligomerize into hexamer. Studies in mammalian cells demonstrated the co-localization of spastin monomers. More interestingly, the walker B motif mutant E442Q showed a dominant negative effect on wild-type spastin. Structural modeling was performed on ATPase domain of spastin. The modeled spastin structure was then used as a framework to classify all the reported spastin mutations in the HSP patients into four different structural/functional categories.

D. V. Krishna Pantakani, Lakshmipuram S. Swapna, Narayanaswamy Srinivasan and Ashraf U. Mannan

Status: Published in Journal of Neurochemistry (Impact factor 4.451), Volume 106 (2008), pp 613-624.

\section{Author contributions to the work:}

1. D. V. Krishna Pantakani: Generation of constructs, protein biochemical studies and immunocytochemical studies. Structural and functional classification of known HSP mutations using modeled spastin structure. Involved in the manuscript preparation.

2. Lakshmipuram S. Swapna: Computational modeling of spastin structure. Involved in the manuscript preparation.

3. Narayanaswamy Srinivasan: Computational modeling of spastin structure. Involved in the manuscript preparation.

4. Ashraf U. Mannan: Conceived and designed the experiments. Interpretated the data and prepared the manuscript. 


\title{
Spastin oligomerizes into a hexamer and the mutant spastin (E4420) redistribute the wild-type spastin into filamentous microtubule
}

\author{
D. V. Krishna Pantakani, * Lakshmipuram S. Swapna, $\dagger$ Narayanaswamy Srinivasan $\dagger$ and \\ Ashraf U. Mannan* \\ *Institute of Human Genetics, University of Goettingen, Goettingen, Germany \\ $\uparrow$ Molecular Biophysics Unit, Indian Institute of Science, Bangalore, India
}

\begin{abstract}
Spastin, a member of the ATPases associated with various cellular activities (AAA) family of proteins, is the most frequently mutated in hereditary spastic paraplegia. The defining feature of the AAA proteins is a structurally conserved AAA domain which assembles into an oligomer. By chemical crosslinking and gel filtration chromatography, we show that spastin oligomerizes into a hexamer. Furthermore, to gain a comprehensive overview of the oligomeric structure of spastin, we generated a structural model of the AAA domain of spastin using template structure of VPS4B and p97/NCP. The generated model of spastin provided us with a framework to
\end{abstract}

classify the identified missense mutations in the AAA domain from hereditary spastic paraplegia patients into different structural/functional groups. Finally, through co-localization studies in mammalian cells, we show that $\mathrm{E} 442 \mathrm{Q}$ mutant spastin acts in a dominant negative fashion and causes redistribution of both wild-type spastin monomer and spastin interacting protein, RTN1 into filamentous microtubule bundles.

Keywords: hereditary spastic paraplegia, hexamer, oligomerization, spastin.

J. Neurochem. (2008) 106, 613-624.
Mutation in the SPAST (SPG4) gene is the single most common cause for hereditary spastic paraplegia (HSP) and accounts for $\sim 40 \%$ of autosomal dominant cases of HSP (Hazan et al. 1999; Fonknechten et al. 2000; Lindsey et al. 2000). The SPG4 subtype is considered as pure form of HSP and clinically characterized by lower limb spasticity, weakness, hyperreflexia, and often mild vibratory sense impairment in the toes (Harding 1983; Fink 2003). The SPAST gene encodes for spastin protein, which belongs to the ATPases associated with various cellular activities (AAA) family of proteins (Patel and Latterich 1998; Hanson and Whiteheart 2005). These ATPases are principally characterized by the AAA domain, often located in the C-terminal part of the protein, whereas the $\mathrm{N}$-terminal part contains recognition sites for substrates and adaptors (Patel and Latterich 1998; Hanson and Whiteheart 2005). All types of mutations are reported in the SPAST gene, including missense, nonsense, splice site mutations, and small insertions/deletions suggesting haploinsufficiency as the pathogenic mechanism (Fonknechten et al. 2000; Lindsey et al. 2000). Interestingly, almost all the missense mutations are located in the C-terminal AAA domain (Fonknechten et al. 2000; Lindsey et al. 2000) and recent studies suggests that these mutations might exert a dominant negative effect on the molecular function of spastin (Errico et al. 2002; Evans et al. 2005).

Numerous studies used different biological systems to determine the molecular function of spastin, in an attempt to elucidate its role in the pathogenesis of HSP. Majority of

Received August 16, 2007; revised manuscript received March 28, 2008; accepted April 2, 2008.

Address correspondence and reprint requests to Dr Ashraf U. Mannan, Institute of Human Genetics, University of Goettingen, HeinrichDueker-Weg 12, D-37073, Goettingen, Germany.

E-mail: amannan@gwdg.de

Abbreviations used: AAA, ATPases associated with various cellular activities; CSU, Contacts of Structural Units; DSP, dithiobis-succinimidyl propionate; GST, glutathione-S-transferase; HSP, hereditary spastic paraplegia; MIT, microtubule interacting and trafficking; MT, microtubule; PBS, phosphate-buffered saline; RTN, reticulon; SDS-PAGE, sodium dodecyl sulfate-polyacrylamide gel electrophoresis; VPS, vacuolar protein sorting. 
these studies suggest that spastin plays a role in microtubule (MT) network dynamics (Errico et al. 2002; McDermott et al. 2003; Evans et al. 2005; Roll-Mecak and Vale 2005; Salinas et al. 2005). Expression of spastin in cultured cells revealed dynamic interaction between spastin and the MT and also partial loss of MT network can be detected in the cells over-expressing spastin (Errico et al. 2002; McDermott et al. 2003; Evans et al. 2005). Lately, it has been shown that spastin possesses direct MT-severing activity (Evans et al. 2005; Roll-Mecak and Vale 2005) and it can also bundle MTs (Salinas et al. 2005). The role of spastin in MTmediated axonal transport is further supported by in vivo studies in Drosophila, zebra fish, and mouse, where knockdown of spastin results in abnormal MT dynamics in the neurons (Sherwood et al. 2004; Trotta et al. 2004; Tarrade et al. 2006; Wood et al. 2006). However, the role of spastin in membrane trafficking and vesicular transport is now emerging. Recently, several studies, including from our group, showed that spastin interacts with different components of vesicular transport process such as chromatin modifying protein 1B (Reid et al. 2005), Zinc finger, FYVE domain containing 27 (Mannan et al. 2006b), atlastin (Evans et al. 2006; Sanderson et al. 2006), and reticulonl (RTN1) (Mannan et al. 2006a). Spastin mediates its interaction with vesicular proteins through its $\mathrm{N}$-terminal region, which contain the MT interacting and trafficking (MIT) domain. The MIT domain is common to a group of proteins involved in endocytosis and intracellular trafficking, such as suppressor of $\mathrm{K}(+)$ transport growth defect 1 and sortin nexin 15 (Ciccarelli et al. 2003). It is also present in spartin, mutations in which cause a complicated autosomal recessive form of HSP, namely, Troyer syndrome (Patel et al. 2002). The fact that spastin contains MIT domain and localizes in punctate vesicular structures in cultured cells; further strengthen its role as mediator of vesicular transport processes.

Despite recent advancement in molecular studies, a comprehensive function of spastin has not yet been elucidated. It appears that spastin is a multifaceted protein with versatile role in cellular events. The functional diversity of spastin can be attributed to different functional regions of spastin such as the nuclear export sequence, nuclear localization sequence, transmembrane motif, MIT, and more importantly the AAA domain as almost all missense mutations reported in spastin are in the AAA domain. The central feature of AAA family is a structurally conserved AAA domain which binds to ATP and assembles into an oligomer. The AAA domain contains Walker A and B motifs and also several other motifs that distinguish it from classic $P$ loop NTPases (Confalonieri and Duguet 1995; Frickey and Lupas 2004). Apart from the AAA domain, these proteins consist of various other domains, which interact with adaptor proteins to generate the structural and functional diversity of the family (Confalonieri and Duguet 1995; Frickey and
Lupas 2004; Hanson and Whiteheart 2005). Majority of the AAA proteins function as molecular machines which disassemble protein complexes. They couple energy derived from ATP hydrolysis to achieve their role by promoting conformational changes or remodeling in target proteins (Patel and Latterich 1998; Hanson and Whiteheart 2005). The phylogenetic analysis based on sequence similarity places spastin into a subfamily called meiotic group of AAA proteins (Frickey and Lupas 2004). The notable members of this group are katanin (p60) (McNally and Vale 1993) and vacuolar protein sorting 4 (suppressor of $\mathrm{K}(+)$ transport growth defect 1) (Yoshimori et al. 2000). Katanin is a MTsevering protein and forms an oligomer in the ATP-bound state (Hartman and Vale 1999). VPS4, which also harbors a MIT domain in its N-terminal region, is involved in vacuolar sorting and endosomal transport. The wild-type VPS4 protein cycles between soluble, inactive low molecular weight complexes and active, membrane-associated doublering structures (10-12 subunits) (Babst et al. 1998; Scott et al. 2005). Based upon sequence similarity it can be postulated that spastin might also form an oligomeric structure to render its function. A very recent study reported that the AAA domain of spastin can assemble into hexamer (White et al. 2007). In their study, the hexameric state of AAA domain of spastin was very unstable as it could not be stabilized even in the presence of slowly hydrolysable ATP $\gamma \mathrm{S}$ and they could only trap the hexamer, when they used the Walker B E442Q mutant form of spastin AAA domain.

In the present study, we used full-length spastin and showed through chemical cross-linking and gel filtration chromatography that wild-type spastin oligomerizes into a hexamer. Our results suggest that N-terminal region of spastin might play a critical role in the formation of a stable hexamer. Furthermore, a computational model of the oligomeric form of spastin was generated, which was used to elucidate the structural basis of the stability of the hexameric structure of spastin.

\section{Materials and methods}

Generation of GST-spastin construct, expression, and purification The human short isoform of spastin initiating from the alternative start codon (M87) was amplified by RT-PCR using a primer pair, M87f, GAATTCATGGCAGCCAAGAGGAGCTCCGGGG and M87r, AAGCTTGTTAAACAGTGGTATCTCCAAAGTCCTTG with Takara Taq polymerase (Takara Bio Inc., Japan) from human brain cDNA (BD Biosciences, Palo Alto, CA, USA). The amplified RT-PCR product was cloned into EcoRI and HindIII sites of pET41a vector (Qiagen, Hilden, Germany). Any possible incorporation of mutation during PCR amplification was excluded by complete sequencing of the construct. The spastin-pET41a construct was transformed into BL21-DE3 strain of Escherichia coli and bacterial culture was grown at $30^{\circ} \mathrm{C}$ until 0.8 optical 
density at $620 \mathrm{~nm}$ was reached then induced with $0.5 \mathrm{mM}$ isopropyl $\beta$-D-1 thiogalactopyranoside for $4 \mathrm{~h}$. The induced bacteria were lysed by sonication either in buffer $\mathrm{A}$ (with $2 \mathrm{mM}$ ATP) or B (with $5 \mathrm{mM} \mathrm{MgCl}$ ) containing $50 \mathrm{mM}$ Tris$\mathrm{HCl}, \mathrm{pH} 8.0,200 \mathrm{mM} \mathrm{NaCl}, 10 \%$ glycerol, $5 \mathrm{mM}$ dithiothreitol, and protease inhibitors. Bacterial debris from protein extract was cleared by centrifugation at $14000 \mathrm{~g}$ for $30 \mathrm{~min}$ at $4^{\circ} \mathrm{C}$. The cleared protein extract was passed through glutathioneagarose column (Sigma, St Louis, MO, USA) pre-equilibrated with buffer $\mathrm{A} / \mathrm{B}$ and washed with 10 column volumes of buffer $\mathrm{A} / \mathrm{B}$. The bound protein was eluted using $10 \mathrm{mM}$ reduced glutathione in either buffer A or B. The glutathione-S-transferase moiety was cleaved from the purified GST-spastin protein by overnight incubation with thrombin enzyme (Sigma) at $4^{\circ} \mathrm{C}$. The cleaved GST was removed from spastin by glutathione-agarose binding.

\section{ATPase assay}

The activity of the purified spastin protein was assayed by using $250 \mathrm{ng}$ of purified protein in a $200 \mu \mathrm{L}$ reaction buffer containing $50 \mathrm{mM}$ Tris- $\mathrm{HCl}, \mathrm{pH} 7.5,5 \mathrm{mM} \mathrm{MgCl}_{2}$, and varying amounts of ATP (Sigma). The reaction was then incubated at $37^{\circ} \mathrm{C}$ for 30 min and was stopped by adding $50 \mu \mathrm{L}$ of malachite green reagent (BioAssay Systems, Hayward, CA, USA) and measured the optical density at $620 \mathrm{~nm}$ on a 96-well plate reader and calculated the amount of inorganic phosphate released by ATPase activity of the spastin from the standard curve. The absorbance of control samples containing the same amount of ATP without the enzyme was subtracted from the overall value of the samples. The $K_{\mathrm{m}}$ and $V_{\max }$ of spastin ATPase was calculated using nonlinear regression analysis (GraphPad Prism 4.0; GraphPad Software Inc., San Diego, CA, USA).

Chemical cross-linking using dithiobis-succinimidyl propionate Chemical cross-linking was performed using homobifunctional, thiol-cleavable dithiobis-succinimidyl propionate (DSP; Pierce Biotechnology, Rockford, IL, USA) with final concentrations ranging from 0.2 to $1 \mathrm{mM}$ in reaction buffer ( $100 \mathrm{mM}$ Phospaste, $\mathrm{pH} 7.2$, and $150 \mathrm{mM} \mathrm{NaCl}$ ) using $5 \mu \mathrm{g}$ of purified spastin protein in total volume of $30 \mu \mathrm{L}$. Reaction mixtures were then incubated at $\sim 25^{\circ} \mathrm{C}$ for $20 \mathrm{~min}$ and stopped by adding $1 \mathrm{M}$ Tris- $\mathrm{HCl}, \mathrm{pH} 8.0$, and non-reducing Laemmli loading dye. The samples were then electrophoresed without boiling on 4-12\% Bis-Tris gradient gel (Invitrogen, Paisley, UK) and transferred onto nitrocellulose membrane (Hybond C-extra; Amersham Bioscience, Bucks, UK) and probed with rabbit polyclonal anti-spastin antibody, 54S1 (kind gift from Professor Elena I. Rugarli).

\section{Gel filtration chromatography and immunoblotting}

For gel filtration chromatography, we used Superdex 200 PC 3.2/30 HPLC column system (Amersham Biosciences); the column bed volume was $2.4 \mathrm{~mL}$. The purified GST-spastin or cleaved spastin (either with ATP or without ATP) was centrifuged at $15700 \mathrm{~g}$ for $10 \mathrm{~min}$ and about $50 \mu \mathrm{g}$ of protein $(1 \mu \mathrm{g} / \mu \mathrm{L})$ was loaded onto the Superdex 200 column, which was pre-equilibrated with either buffer A or B. The protein elution was performed at a flow rate of $40 \mu \mathrm{L} /$ $\min$ and about 60 fractions $(40 \mu \mathrm{L}$ each) were collected. The molecular weight of native spastin and GST-spastin oligomer was extrapolated from standard curve plotted with the values obtained from the protein molecular weight of standard markers (Amersham Bioscience). The protein fractions were resolved on $10 \%$ sodium dodecyl sulfate-polyacrylamide gel electrophoresis (SDSPAGE) and transferred onto nitrocellulose membrane (Amersham Bioscience). Western blot was performed with polyclonal antispastin antibody, 54S1 as described previously (Errico et al. 2004).

Modeling of tertiary and quaternary structure of the AAA domain of spastin

The complete AAA domain sequence of spastin was searched against PDB (Berman et al. 2000) using the BLAST program (Alstchul Lab, NCBI, Bethesda, MD, USA, http:www.ncbi.nlm. nih.gov/blast) (Altschul et al. 1997), with default parameters, to identify closely related homologs of spastin with known 3-D structure. The $\Lambda \Lambda \Lambda \Lambda$ TPase domain of spastin was modeled on the basis of the tertiary structures of two templates (PDB codes: 1xwi and 1s3s) (Dreveny et al. 2004; Scott et al. 2005) using MODELLER version 8.0 (Sali Lab, UCSF, San Francisco, CA, USA, http:// www.salilab.org/modeller) (Sali 1995). The generated models were energy minimized using the Kollman united atom force field in SYBYL (Tripos Inc., St. Louis, MO, USA) to ensure acceptable geometry and to relieve short contacts. The overall fit of the sequence to the template was checked using Verify 3D (Luthy Lab, University of California, CA, USA; http://www.nihserver.mbi. ucla.edu/Verify_3D) (Luthy et al. 1992). The copies of modeled tertiary structure were assembled to form a hexameric quaternary assembly on the basis of the hexameric template (1s3s). This modeled quaternary structure was energy minimized using SYBYL. The interfacial residues between the monomers were extracted using the Contacts of Structural Units (CSU) program (Sobolev Lab, Weizman Institute of Sciences, Israel, http://www.bip. weizmann.ac.il/oca-bin/lpcesu) (Sobolev et al. 1999) and the crucial residues were short listed by manual inspection and used for further analysis.

Generation of constructs, immunoprecipitation, and immunocytochemical analysis

The generation of spastin-pCS2, spastin-pEGFP, and RTN1-pQM constructs were described previously (Mannan et al. 2006a). To introduce the E442Q and R499C missense mutations in spastin, we used spastin-pEGFP plasmid DNA as template and the mutation was introduced by using the QuikChange site-directed mutagenesis kit (Stratagene, La Jolla, CA, USA). The presence of the point mutation was confirmed by DNA sequencing. The primers used to generate the point mutations were: E442Qf, CCTTCTATAATTTTTATAGATCAAGTTGATAGCCTTTTGTGTG; E442Qr, CACACAAAAGGCTATCAACTTGATCTATAAAAATTATAGAAGG; R499Cf, GATGAGGCTGTTCTCAGGTGTTTCATCAAACGGGTATATG; R499Cr, CATATACCCGTTTGATGAAACACCTGAGAACAGCCTCATC. Immunoprecipitation assay was performed as described previously (Mannan et al. 2006a). For immunocytochemistry analysis, the NIH3T3 cells were seeded into two chamber culture slides (BD Falcon, Palo Alto, CA, USA) for transfection. After $24 \mathrm{~h}$, cells were co-transfected with the c-Myc-spastin and Gfp-E442Q-spastin/ Gfp-R499C-spastin constructs using Lipofectamine 2000 reagent (Invitrogen). Following $24-30 \mathrm{~h}$ of transfection, the cells were 
washed two times with phosphate-buffered saline (PBS) and fixed in $4 \% p$-formaldehyde in PBS for $30 \mathrm{~min}$. The transfected cells were permeabilized and blocked by incubating in blocking solution [30\% goat serum (Invitrogen), $20 \mathrm{mM}$ phosphate buffer, $\mathrm{pH} 7.2$, $450 \mathrm{mM} \mathrm{NaCl}$, and $0.3 \%$ Triton X-100] for $30 \mathrm{~min}$. In the next step, the cells were incubated with a c-Myc antibody (Dunn Labortechnik, Asbach, Germany) in a dilution of $1: 200$ for $2 \mathrm{~h}$ at $\sim 25^{\circ} \mathrm{C}$. Thereafter, cells were washed three times for $10 \mathrm{~min}$ each with PBS and then incubated with anti-rabbit IgG conjugated with $\mathrm{Cy} 3$ (Sigma) at a dilution of $1: 100$ for $1 \mathrm{~h}$ at $\sim 25^{\circ} \mathrm{C}$. Finally, the stained cells were washed again three times for 10 min each with PBS and let to air dry. The culture flask was detached from the glass slide according to the manufacture's instruction and VectaShield solution (Vector Laboratories, Burlingame, CA, USA) was applied before mounting with a coverslip. Slides were observed using a confocal microscope (Olympus, Hamburg, Germany) and images were processed by the Cell-f program (Soft Imaging System, Muenster, Germany).

\section{Results}

Expression and purification of short isoform of spastin Spastin has many isoforms, the long isoform and a more abundant nuclear/cytoplasmic short isoform encoded by an alternative start site, corresponding to residue 87 of fulllength spastin (long isoform) (Claudiani et al. 2005). The short isoform is likely to be the most soluble form in the cytoplasm as it lacks the putative transmembrane motif; therefore, a high level of expression of this isoform can be achieved in bacteria. For these reasons, we chose short isoform of spastin (referred as spastin) for our studies and cloned it into bacterial expression vector. Analysis of recombinant spastin protein, which was affinity purified from bacterial protein extract and resolved by SDS-PAGE revealed a high level of expression in soluble fraction

(a)

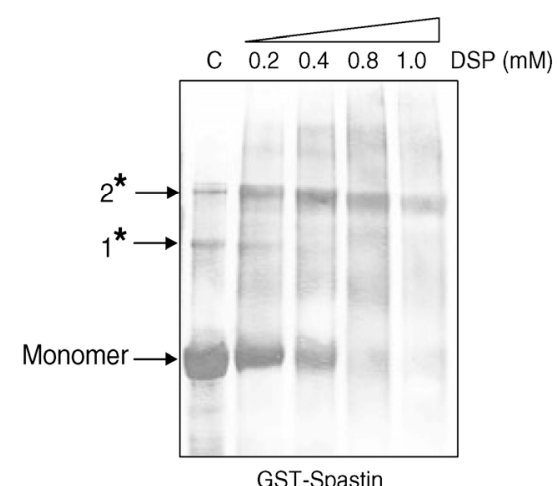

(b)

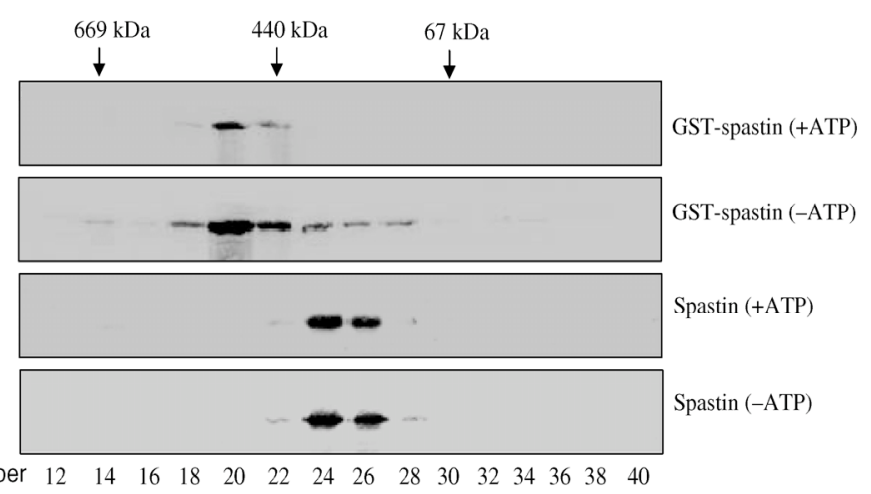

Fig. 1 Oligomerization of spastin. Chemical cross-linking of spastin by dithiobis-succinimidyl propionate (DSP). Purified GST-spastin was subjected to chemical cross-linking with DSP and was resolved in SDS-PAGE under non-reducing condition and transferred to nitrocellulose membrane, western blot was performed with spastin polyclonal antibody. Asterisks denote higher order cross-linked oligomeric spastin. Two major forms of oligomeric spastin were detected after cross-linking, probably an intermediate form designated as asterisk 1 and predominant hexameric form represented as asterisk 2 . The formation of hexameric spastin was dependent upon the concentration of DSP, at high concentration of DSP $(1 \mathrm{mM})$ only hexameric form of spastin exists. Interestingly, even in the absence of DSP cross-linking (denoted as lane C), besides predominant monomeric spastin, a fraction of both higher order oligomeric spastin were detectable (a). HPLC gel filtration chromatography. The purified GST-spastin or cleaved spastin (either with ATP or without ATP) was subjected to gel filtration chromatography on Superdex 200 HPLC column. The aliquot of eluted fractions were immunoblotted with anti-spastin antibody. The elution peaks for marker proteins (in $\mathrm{kDa}$ ) are indicated across the top of the immunoblot. The number of the eluted fraction in the chromatography is represented below the immunoblot (b). 
(Supplementary Fig. S1a) and the purified recombinant spastin protein was enzymatically active as confirmed by ATPase assay, with a $K_{\mathrm{m}}$ value of $1.497 \pm 0.24$ and $V_{\max }$ of $0.2874 \pm 0.023$ (Supplementary Fig. S1b).

\section{Wild-type spastin forms a hexamer}

Majority of the AAA proteins function as oligomer. To determine whether spastin can also assemble as an oligomer, we first cross-linked purified GST-spastin with thiol-cleavable chemical cross-linker DSP. Resolving of cross-linked GSTspastin by SDS-PAGE suggested that spastin is able to form an oligomeric structure (Fig. 1a). We observed an intermediate oligomeric form as well as a hexameric form of spastin in our cross-linking studies, which was dependent on the concentration of DSP (Fig. 1a). At higher concentration of DSP, hexamer was the most prominent form of spastin (Fig. la).

To find out the precise oligomeric state of spastin, we next analyzed GST-spastin by gel filtration chromatography. As most AAA proteins are known to assemble into oligomers in an ATP-dependent manner, but remain monomeric in the absence of nucleotide, the first set of gel filtration assay was performed in presence of ATP. The GST-spastin purified in presence of ATP was subjected to gel filtration chromatography and the eluted fractions were analyzed by western blot using spastin antibody. Based upon our gel filtration analysis, it appears that the GST-spastin forms a hexamer as it eluted at a native size of $\sim 530 \mathrm{kDa}$ (monomer is $\sim 88 \mathrm{kDa}$ ), which is consistent with the proposed hexameric state of GST-spastin (Fig. 1b). To investigate whether the oligomeric state of spastin is ATP dependent or independent, we performed gel filtration assay in an ATP depleted condition. Analysis of elution profile revealed GST-spastin in absence of ATP also eluted at a native size of $\sim 530 \mathrm{kDa}$; however, spastin eluted in multiple fractions, showing a diffuse pattern of elution (Fig. 1b). To exclude any possibility of artifact generated due to GST moiety, we cleaved the GST tag from recombinant spastin and performed gel filtration assay with GST-free spastin protein in the presence and absence of ATP. The elution pattern of the gel filtration assay revealed that spastin forms a hexameric structure, which was eluted at native size of $\sim 350 \mathrm{kDa}$ (Fig. 1b). ATPase assay was performed with all the eluted fractions and fractions containing spastin oliogmers were enzymatically active (data not shown).
Fig. 2 Structural models of spastin AAA domain. The tertiary structures of $\mathrm{P} 97 / \mathrm{VCP}$ (a), VPS4B (b), and modeled AAA ATPase domain of spastin (c) are shown as ribbon diagrams. Regions undergoing conformational change are colored blue and interacting residues between the monomers are colored magenta in all the three structures. The beta domain of VPS4B is marked in green (b). The N-terminal beta strand $\left(\beta^{\prime}\right)$ unique to meiotic AAA ATPases is highlighted in brown (b and c). The C-terminal strand unique to meiotic AAA ATPases is painted olive green in ( $b$ and $c$ ). The structures of template hexameric ring (d) and hexameric model of spastin AAA domain (e) are shown as ribbon structure. Each monomer is labeled in different color. The key interacting residues of both surfaces are depicted as spheres, in red and blue colors, respectively. (a)

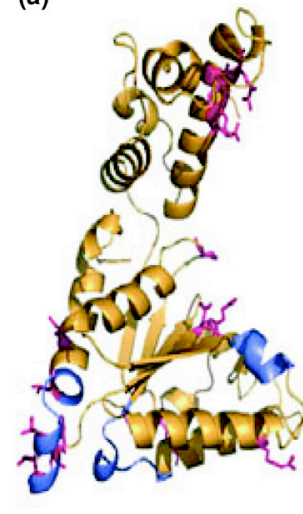

p97NCP (1s3s)

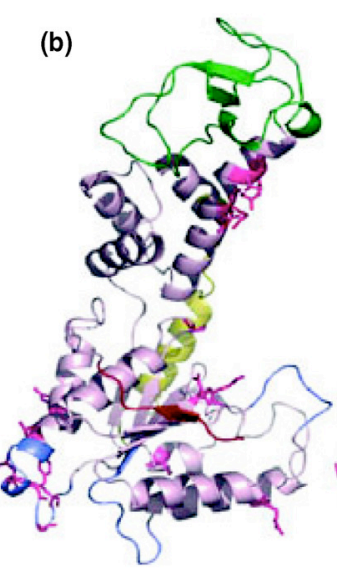

VPS4B (1xwi) (c)

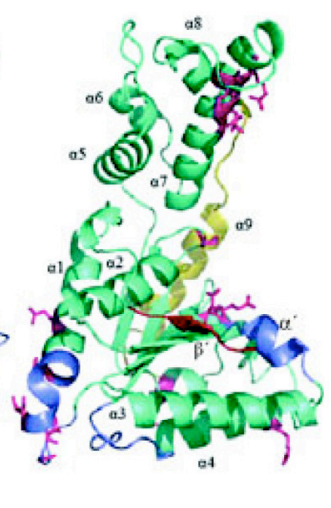

Model of spastin

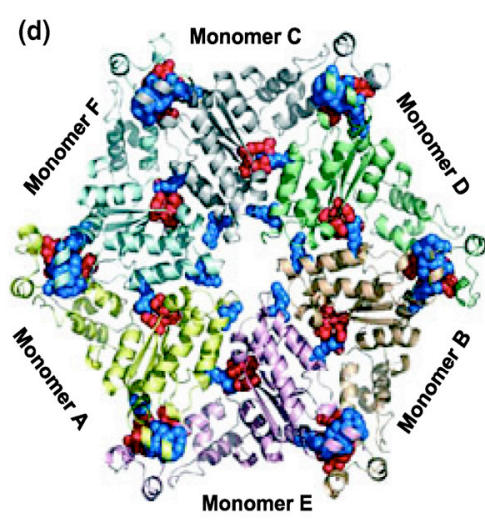

Template hexamer (p97NCP)

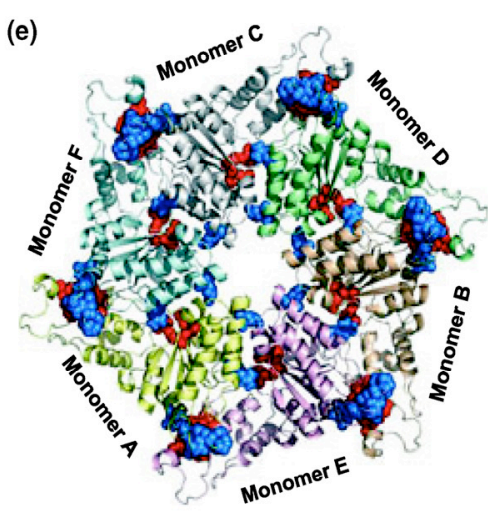

Model of hexameric spastin 
Modeled tertiary and quaternary structure of the AAA ATPase domain of spastin

Overview of modeled tertiary structure

A typical AAA ATPase domain consists of two subdomains, an $N$-terminal $\alpha / \beta$ domain followed by a smaller four-helix bundle. Our model shows that this tertiary structure is conserved in spastin (Fig. 2c). The Walker A and B motifs (355-GKT-357 and 409-DE-410) and the arginine finger (466-RR-467) of the second region of homology are the key sequence motifs which characterize the nucleotide binding site of the AAA ATPase domain. All these sequence motifs are conserved in the primary structure of spastin (Supplementary Fig. S2).

\section{Comparison of AAA ATPase domains of spastin, VPS4B, and $p 97 / V C P$}

The VPS4B protein (PDB code: 1xwi) (Scott et al. 2005), crystallized as a monomer, is the closest homolog of spastin with a crystal structure available (Fig. $2 \mathrm{~b}$ ) and show $50 \%$ sequence identity over the length of the domain (Fig. S2). The sequence and structural similarity between the VPS4B and spastin domains is quite high in the regions of secondary structures, whereas the loops show some variations (Fig. 2b and $c$ and Fig. S2). The main difference is the absence of beta domain at the C-terminus of VPS4B, which is a feature of all vacuolar sorting proteins (Fig. 2b). The closest homolog of known structure which forms a hexamer is D1 domain of p97/VCP (PDB code: 1s3s) (Dreveny et al. 2004) (Fig. 2d). The AAA ATPase domain of spastin shares 36\% sequence identity over the entire length with murine p97/ VCP (Fig. S2). The overall tertiary structure of modeled spastin and p97/VCP domains (Fig. 2a and c) is similar except for the presence of a N-terminal $\beta$ sheet and $\mathrm{C}$ terminal $\alpha$ helix, which are characteristic of meiotic AAA ATPases such as spastin and VPS4B (Fig. 2a-c). The amino acid sequence of C-terminal region ( $\sim 10$ residues) of spastin is quite divergent from the other AAA ATPase domains (Fig. 2c).

\section{Overview of quaternary structure of the spastin AAA ATPase domain}

The hexameric model of spastin, which was generated based on the tertiary structure of VPS4B (Scott et al. 2005) revealed major short contacts at the interface regions. Therefore, we used the tertiary structures of both p97/VCP (Dreveny et al. 2004) and VPS4B (Scott et al. 2005) as joint templates for generating the tertiary structure of AAA ATPase domain of spastin with inter-monomer regions extrapolated from the topologically equivalent regions of p97/VCP. Using such a tertiary structural model we were able to generate an acceptable hexameric structure using p97/ VCP as the template (Fig. 2e). The key interacting residues between the monomers of the AAA ATPase subunits of $\mathrm{p} 97 /$
$\mathrm{VCP}$, extracted by the program CSU, are also conserved in VPS4B and spastin. The interacting partner amino acids for the key interacting residues as determined by CSU for both template and model are also reasonably conserved, which are summarized in supplementary Tables S1 and S2.

Furthermore, we classified known sets of HSP missense mutations identified in the AAA domain from the Human Gene Mutation Database Professional release 7.1 (http:// www.biobase.de/hgmd/pro/start.php) into different structural/functional groups based upon the spastin model structure. We placed these mutations into four categories namely, active site, pore loop structure, monomer interface residues, and other mutations (Fig. 3a-k and Table S3).

Interaction of spastin monomers in the mammalian cells To investigate whether spastin also forms an oligomeric structure under physiological condition, we decided to study interaction between differentially tagged spastin proteins in NIH3T3 cells. For co-immunoprecipitation and co-localization experiments, we utilized previously generated mammalian expression constructs in which spastin was tagged with c-Myc and Gfp moiety (Mannan et al. 2006a). We used a long isoform of spastin lacking exon 4 in these constructs because it localizes to punctate vesicles, which are distributed around perinuclear region of the cell displaying physiological relevance (Mannan et al. 2006a). For coimmunoprecipitation assay, the NIH3T3 cells were cotransfected with c-Myc-spastin and Gfp-spastin constructs. The total protein extract from co-transfected cells were immunoprecipitated with c-Myc antibody and subsequent, western blot analysis with Gfp antibody revealed a band of the size $\sim 91 \mathrm{kDa}$ corresponding to Gfp-spastin (Fig. 4a). However, Gfp-spastin band was undetectable in immunoblot when c-Myc antibody was omitted from the immunoprecipitation reaction (Fig. 4a). Next, we attempted to determine the interaction between wild-type spastin and mutant spastin protein. For these studies, we generated E442Q and R499C mutation in the AAA domain of Gfp-spastin protein (referred further as E442Q-spastin and R499C-spastin). The E442 residue localizes to conserve Walker B motif of AAA domain and this mutation allow ATP binding but prevent its hydrolysis (Evans et al. 2005). The R499C is a disease causing mutation, it constitutes the arginine finger of AAA domain and this mutant protein is unable to bind ATP. Co-immunoprecipitation studies with differentially tagged mutant and wild-type spastin proteins confirmed that both the mutant spastin proteins (E442Q and R499C) were able to associate with wild-type spastin in cells (Fig. 4b-c).

\section{E4420 mutant spastin redistributes wild-type spastin and} RTN1

We next attempted to determine the physiological relevance of interaction between spastin monomers by co-localization studies in mammalian cells. The NIH3T3 cells co-expressing 
Fig. 3 Structural categorization of known HSP mutations. The HSP missense mutations identified in the AAA domain of spastin from database were categorized into four classes based upon the model of spastin. The first groups of mutations are in active site $(a-c)$, the consensus amino acid residues are highlighted in red in the tertiary structure of spastin (a), the known HSP mutations from patients are shown as red ball and stick in tertiary and quaternary structure of spastin (b and c). The conserved pore loop residues are marked as green ribbon in spastin structure (d), the known HSP mutated residues categorized as pore loop mutations are depicted as green ball and stick in spastin monomer and hexamer (e and f). The key interacting residues between monomers required for oligomerization are shown in blue color in the tertiary structure of spastin (g), furthermore, the known set of HSP mutations in this group are also shown in the spastin monomer and hexamer (h and i). The HSP mutations, which could not be classified in above groups was designated as the other class of mutations. Labeling of these mutated residues in the modeled spastin structure revealed they can further be grouped in four clusters, which are labeled as magenta, orange, yellow, and light blue colors ( $\mathrm{j}$ and $\mathrm{k}$ ).

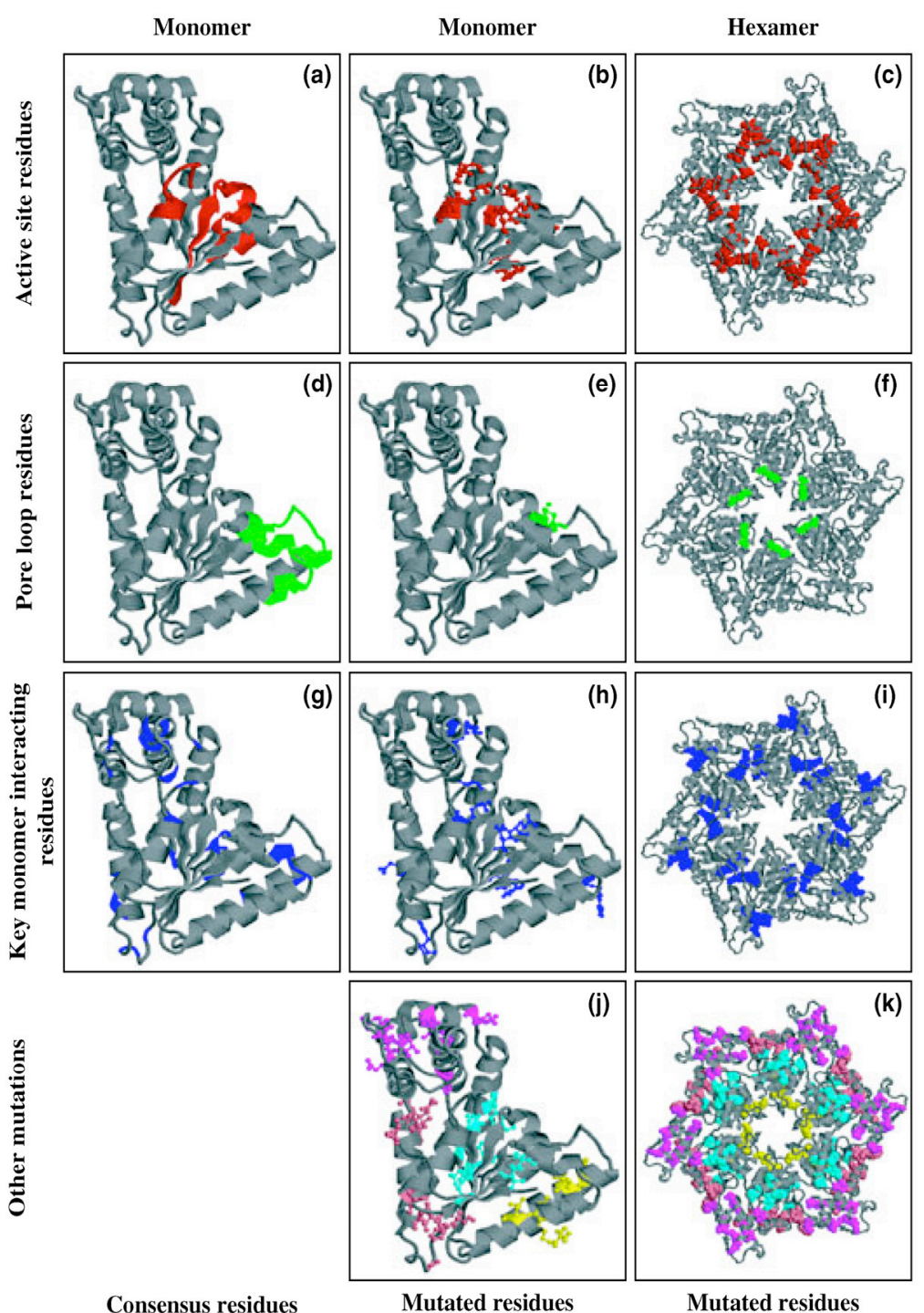

Consensus residues (c) wild-type c-Myc-spastin and Gfp-spastin showed a strong co-localization in endosomal vesicles thus validating the interaction between the spastin monomers (Fig. 5a-d). After that we examined whether wild-type spastin also co-localizes with ATPase defective forms of spastin. The E442Q-spastin showed a filamentous expression pattern in the cytoplasm (Fig. 5e) and it was striking to observe that the expression of wild-type c-Myc-spastin was also filamentous in the same cell (Fig. 5f). The E442Q-spastin caused redistribution of wild-type spastin from punctate vesicles to abnormal filaments decorating MT (Fig. 5e-h). The R499C-spastin showed expression in punctate vesicles (Fig. 5i) and coexpression with wild-type c-Myc-spastin demonstrated strong co-localization in endosomal vesicles (Fig. 5i-1). Next, we evaluated possible effect of E442Q-spastin on intracellular distribution of RTN1, a spastin interacting protein (Mannan et al. 2006a). RTN1 is expressed in vesicles arising from endoplasmic reticulum and endosome. Coexpression of E442Q-spastin and E2-RTN1 in NIH3T3 cells resulted in redeployment of E2-RTN1 to filamentous MTs in the cytoplasm, where it shows co-localization with mutant spastin (Fig. 5m-p).

\section{Discussion}

In the present study, we determined the oligomeric state of spastin by cross-linking and gel filtration chromatography. Spastin forms intermediate state oligomeric structures, which is independent of nucleotide binding; however binding to ATP stabilizes the hexamer form of spastin. Next, we modeled tertiary and quaternary structure of the AAA domain of the spastin based on template crystal structures of VPS4B monomer and p97/VCP hexamer. The structural model of spastin provided us with a framework to determine 


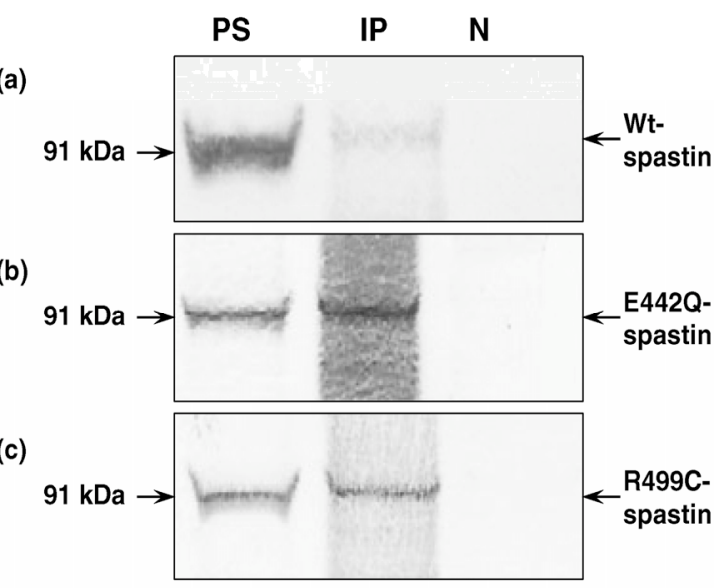

Fig. 4 Immunoprecipitation assay. To determine the interaction be tween spastin monomers, ex vivo immunoprecipitation assay was performed. The soluble total protein extract from NIH3T3 cells cotransfected with c-Myc-spastin and Gfp-spastin constructs was immunoprecipitated with c-Myc antibody and subsequently western blot was performed with Gfp antibody. Lane 1 (PS), corresponds to standard protein lysate, which serves as a control for transfection efficiency; lane $2(\mathrm{IP})$, immunoprecipitated fraction; lane $3(\mathrm{~N})$, mock precipitation was performed without c-Myc antibody. A band sized $91 \mathrm{kDa}$ corresponding to Gfp-spastin can be seen in lane PS and lane IP but not in lane N (a). Similarly, when immunoprecipitation was carried out between wild-type c-Myc-spastin and mutated Gfp-spastin (E442Q-spastin), c-Myc-spastin was able to immunoprecipitate E442Q-spastin (b). Also interaction between c-Myc-spastin and R499C-spastin could be confirmed by immunoprecipitation assay (c)

the residues forming active site, loop structures and monomer-monomer interface residues necessary for oligomerization. Finally, through co-localization studies in mammalian cells, we show E442Q mutant spastin function in dominant negative fashion and causes redistribution of both wild-type spastin monomer and spastin interacting protein RTN1 into MT bundles.

Spastin is a multifaceted protein and exists in several isoforms and probably its different molecular functions are mediated through its various isoforms. The two major isoforms of spastin are the long form starting from M1 codon and an N-terminal truncated (1-86 amino acid) form encoded from M87 named the short isoform (Claudiani et al. 2005). The spliced variants lacking exon 4 of either of these isoforms appears to be more relevant for endogenous function as no mutation out of over 220 mutations reported were detected in exon 4 . There are conflicting reports concerning physiological relevance of long and short isoform of spastin. A recent study claims that short isoform is predominantly expressed in brain and retains all the physiological function (Salinas et al. 2005). Interestingly, it is also reported that the long isoform is enriched in spinal cord when compared with other tissues (Claudiani et al. 2005 and Solowska et al. 2008); therefore, it is of direct importance for the pathogenesis of HSP. Further studies requiring specific targeting of either of the isoforms of spastin will be necessary to elucidate the isoform specific physiological function of spastin. In the current study, we used the short isoform of spastin for experimental simplicity as this isoform lacks the hydrophobic domain, therefore, it is likely to be more soluble and tendency to form aggregates is minimal. In addition the role of $\mathrm{N}$-terminal region of spastin on the stability of possible oligomeric structure driven by the AAA domain can only be emphasized when the studies are carried out with a full-length form of the protein. The rationale behind using a full-length isoform was further highlighted with the fact that affinity of full-length spastin (long isoform) isoform $\left(K_{\mathrm{m}}=0.45 \pm 0.07 \mathrm{mM}\right)$ for its substrate ATP is much higher than that of AAA domain of spastin $\left(K_{\mathrm{m}}=2.2 \pm 0.63 \mathrm{mM}\right)$ (White et al. 2007).

The first indication that spastin exists as a higher order oligomeric structure was derived from DSP chemical crosslinking studies. Interestingly, in absence of DSP, a minority of spastin fraction was in oligomeric state and we observed two oligomeric fractions of spastin corresponding to a lower order intermediate form and a higher order, perhaps equivalent to hexameric form. At higher concentration $(1.0 \mathrm{mM})$ of DSP, the predicted hexamer was the predominant form of spastin and both monomeric and intermediate forms were almost negligible suggesting that spastin oligomerization was DSP concentration dependent. To determine the precise number of spastin monomers which constitute the higher order spastin oligomeric structure, we performed gel filtration assay. We demonstrated that approximately six monomers comprise the oligomer thus spastin monomers can assemble into a hexamer. However, in contrast to many AAA proteins, which require ATP/ADP to form oligomers, spastin oligomerization state was independent of nucleotide binding state. Nevertheless, it appears that binding of ATP favors the hexameric conformation, as in gel filtration assay in absence of ATP the oligomeric spastin eluted in a broad spectrum of fractions suggesting spastin exists in numerous oligomeric states. In presence of ATP, spastin hexameric state is stabilized as elution pattern in gel filtration became much narrower and eluted fraction molecular weight corresponded to hexameric spastin. It can be postulated that in absence of ATP, a steady state equilibrium exists between monomer $\leftrightarrow$ intermediate oligomer(s) $\leftrightarrow$ hexamer spastin. However, binding of ATP drives this equilibrium towards hexameric state. The steady state equilibrium model for spastin is also supported by chemical cross-linking study as a minor fraction of intermediate and higher (hexamer) form of spastin could be detected even under non-cross-linking condition. The nucleotide binding induced conformation change from intermediate state to hexamer is also reported for $\mathrm{ClpB}$, a bacterial AAA + ATPase, which is a member of a multichaperone system (Goloubinoff et al. 1999; Motohashi et al. 1999; Zolkiewski 1999). In the nucleotide free- 

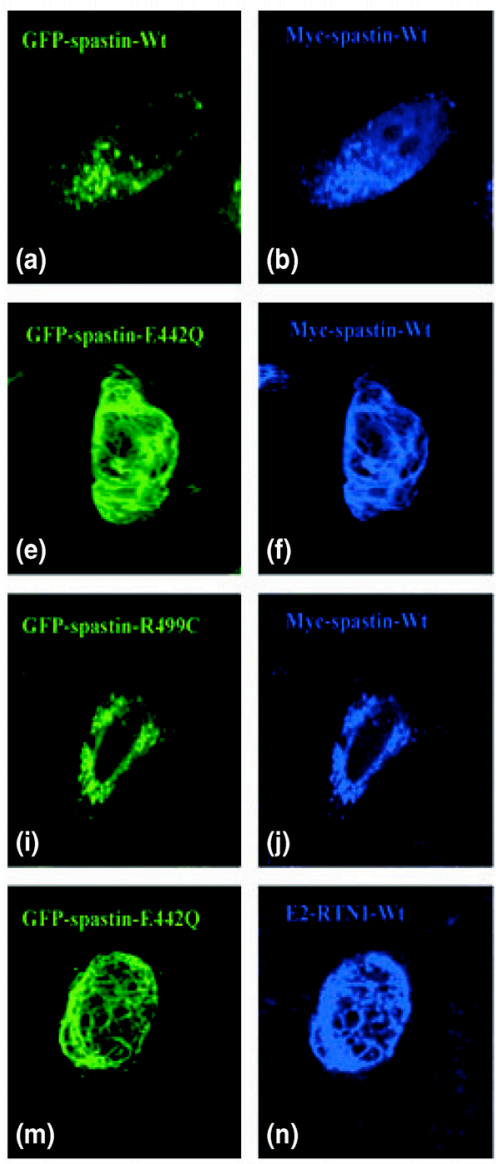

Fig. 5 Co-localization of spastin monomers and RTN1 in mammalian cells. NIH3T3 cells were transiently co-transfected with c-Myc-spastin and Gfp-spastin. Both Gfp-spastin (a) and c-Myc-spastin (b) showed expression in the endosomal vesicle (c) arising from perinuclear region of cell. Overlay of image $(\mathrm{a}-\mathrm{c})$ showed strong co-localization of c-Mycspastin and Gfp-spastin (d). Co-expression of E442Q mutant Gfp-spastin (E442Q-spastin) and wild-type c-Myc-spastin (e-h). E442Q-spastin, showed typical filamentous expression pattern (e), however in the same cell, wild-type c-Myc-spastin expression was

state, ClpB undergoes reversible self-association that involves protein concentration-dependent population of monomers, heptamers, and intermediate-size oligomers (Akoev et al. 2004). In contrast, binding to ATP $\gamma \mathrm{S}$ as well as ADP stabilizes the hexameric form of ClpB (Akoev et al. 2004). Furthermore, p97/VCP, an AAA family protein, which acts as a molecular chaperone in diverse cellular events, can form hexamers without the addition of nucleotide (Wang et al. 2003). The p97/VCP contains an N-terminal substrate-binding domain and two conserved ATPase domains D1 and D2, which are separated from N-terminal domain by a linker motif. This nucleotide-independent hexamerization only requires an intact D1 and the linker sequence of $\mathrm{p} 97 / \mathrm{VCP}$ (Wang et al. 2003). Besides, it is also reported that spastin MT-associated protein like activity, which promotes MT bundling is also independent of
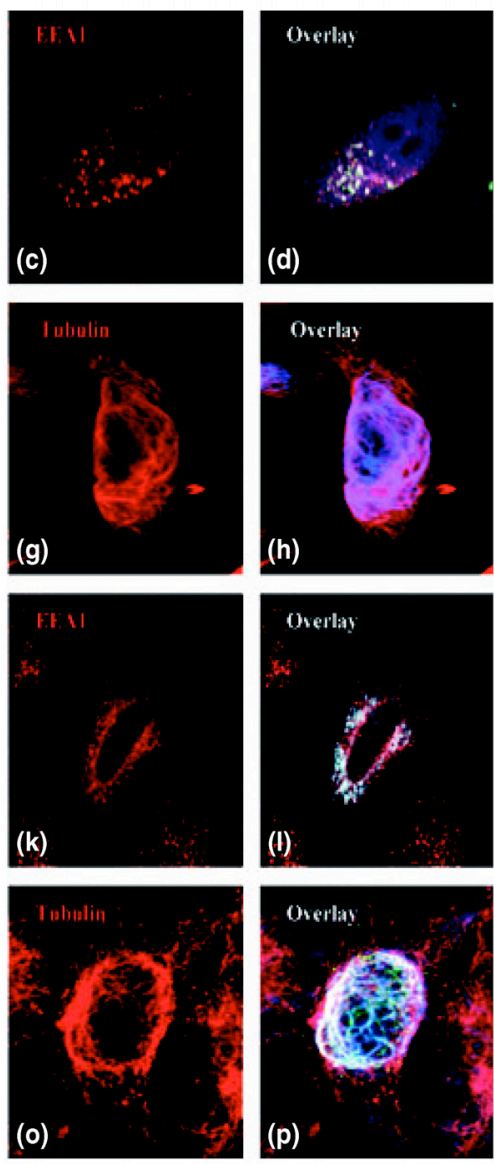

redistributed from expected punctate vesicles to filamentous pattern (f). Overlay images (e and f) with microtubule staining (g) revealed complete co-localization of wild-type and mutant spastin (h). Wild-type c-Myc-spastin and mutant R499C-spastin show co-expression in endosomal vesicles (I-L), R499C-spastin (i), c-Myc-spastin (j), EEA1 (k), and overlay (l). Furthermore, co-expression of E442Q mutant spastin and E2-RTN1 (spastin interacting protein) led to misexpression of E2RTN1 from punctate vesicles to microtubule filament ( $m-p)$, E442Qspastin (m), E2-RTN1 (n), EEA1 (o), and overlay (p).

nucleotide binding (Salinas et al. 2005). Recently, a study reported that the AAA domain of spastin can assemble into hexamer (White et al. 2007). The hexameric state of AAA domain of spastin was very unstable as it could not be stabilized even in the presence of slowly hydrolysable ATP $\gamma \mathrm{S}$ and they could only trap the hexamer when they used the Walker B E442Q mutant form of spastin AAA domain. The discrepancy between our observation and this report could be due to the fact that we used entire short isoform of spastin form and they used deleted and modified AAA domain. The full-length spastin is a true representative of endogenous protein and perhaps the $\mathrm{N}$-terminal domain stabilizes the oligomeric state of spastin as also shown for p97/VCP protein in which N-terminal linker motif is necessary for hexamerization (Wang et al. 2003). However, our study was not able to conclusively address or exclude the 
possibility of the trace amount of bacterial ATP, which might be bound to spastin during purification and gel filtration.

To gain a comprehensive overview of the oligomeric structure of spastin, we generated a model of the AAA domain of spastin using template structures of VPS4B (Scott et al. 2005) and p97/VCP (Dreveny et al. 2004). The modeled tertiary structure of spastin preserves the overall structural architecture of a typical AAA domain, which is defined by two central domains: an amino-proximal $\alpha / \beta$ domain and a smaller four-helix bundle. Additionally, the modeled spastin also consists of an $\mathrm{N}$-terminal $\beta$ sheet $\left(\beta^{\prime}\right)$ and a C-terminal $\alpha$ helix, which is also reported in VPS4B (Babst et al. 1998; Scott et al. 2005). These structural variants appear to be characteristics of the meiotic clade of AAA ATPases, which also include fidgetin and katanin (p60) (Frickey and Lupas 2004) and in this respect, the meiotic subgroups appears distinct from other AAA proteins. However, several structural features are different among structures used as templates and spastin model. For example, comparative analysis indicates that the region of monomeric VPS4B (Scott et al. 2005), aligning with $\alpha^{\prime}$ helical region of the hexameric p97/VCP crystal structure (Dreveny et al. 2004), adopts a different conformation. Comparison of the monomeric and hexameric crystal structures of the homologs suggests that regions $\alpha^{\prime}$ and $\alpha 1$ helix undergo conformational change between monomeric and hexameric forms. For these reasons, the $\alpha^{\prime}$ and $\alpha 1$ regions in the tertiary structural model of spastin were generated using the equivalent regions of p97/VCP crystal structure. Furthermore the loop structures of spastin model are variable compared with the templates.

Most AAA ATPases function as oligomeric rings, usually a hexamer and previously, p97/VCP was crystallized as a hexamer with bound ADP (Dreveny et al. 2004). The similarity between spastin and $\mathrm{p} 97 / \mathrm{VCP}$ allowed us to model oligomeric state of spastin by simply overlaying six spastin subunits onto hexameric p97/VCP, which produced a feasible model with no major steric clashes. In fact, most residues that make up the protein-protein interfaces between monomers are conserved between $\mathrm{p} 97 / \mathrm{VCP}$ and spastin, indicating that the two hexameric assemblies are likely to be conserved Taken together, our gel filtration and modeling data, it favors the idea that spastin oligomerizes into hexameric ring. The C-terminal end helix of spastin, which appears to be the property of meiotic clade, in VPS4B forms an exposed annulus on one side of the hexameric ring, which is suggested to mediate ring dimerization and thereby stabilize a double-ring structure (Scott et al. 2005). Therefore, higher order hexamer possibly a dodecamer structure for spastin cannot be completely ruled out.

The model of spastin provides us with a framework to classify the identified missense mutations in the AAA domain from HSP patients into structural/functional groups. We categorized the known missense mutations reported in database as active site, monomer-monomer interaction, pore- loop and unknown structural group of mutations. The present classification of missense mutations in AAA domain will facilitate our understanding of molecular function of different subdomains of spastin. In future, it will enable us to classify an identified sequence variant in a HSP patient as disease causing mutation with greater level of confidence. In addition, more information concerning the functional implication of identified mutation can be deciphered from the spastin model.

However, recently (while our paper was in revision), a crystal structure of Drosophila spastin AAA domain was reported (Roll-Mecak and Vale 2008), which also suggest that spastin forms a hexamer ring with a prominent central pore and six radiating arms that may dock onto the MT. Their study revealed that the spastin apoenzyme exists in equilibrium between monomeric $\leftrightarrow$ dimeric (intermediate) forms, whereas binding of ATP favors the hexameric conformation, which was comparable to our findings. Furthermore, findings from Roll-Mecak and Vale regarding the role of N-terminal region (the linker and MIT domain) acting as stabilizing factor for the hexameric structure is supportive of our data, which also point out that $\mathrm{N}$-terminal region is required for a stable formation of hexamer.

Next, we went on to validate that spastin indeed functions as oligomer in the mammalian cells by ex vivo immunoprecipitation and co-localization studies. Remarkably, when we used ATPase-defective E442Q mutant of spastin, which binds to ATP, however is unable to hydrolyze it (Evans et al. 2005), in ex vivo co-localization study, we observed redistribution of wild-type spastin from punctate vesicles to filamentous pattern, where it co-localized with E442Q. Also, co-expression of the E442Q mutant spastin with RTN1, a previously characterized spastin interacting protein (Mannan et al. 2006a) caused misexpression of RTN1 to filament instead of typical punctate vesicular expression. Previously, a similar redistribution effect of mutant spastin was also reported between wild-type/K338R-spastin (Errico et al. 2002), atlastin/E442Q-spastin (Evans et al. 2005) and atlastin/K338R-spastin (Sanderson et al. 2006). Furthermore, several known disease causing mutations shows filamentous pattern of expression, such as $\mathrm{I} 344 \mathrm{~K}, \mathrm{~N} 386 \mathrm{~K}, \mathrm{~K} 388 \mathrm{R}$, and R424G (Ki et al. 2002; Errico et al. 2002; McDermott et al. 2003; Evans et al. 2005). These mutations most likely depict the substrate entrapped state of the enzyme and most likely will render a dominant negative function by causing redistribution of wild-type spastin.

Mutational screen have identified over 224 different mutations in spastin, which include all types of mutations such as missense, nonsense, splice site mutations, and insertions/deletions. This wide spectrum of mutation led to a generalized perception that underlying cause for spastinassociated HSP is haploinsufficiency and degeneration of axons results from failure to achieve a threshold level of spastin for normal MT severing. However, presence 
of another MT-severing protein, P60-katanin, which shows an overlapping expression pattern with spastin and is expressed at much higher level compared with spastin (Yu et al. 2005 and Solowska et al. 2008) further demur the 'loss of function model' for spastin. Moreover, a knockout mouse lacking functional spastin showed only late and mild form of degeneration and no detectable abnormalities during development (Tarrade et al. 2006). Contrarily, in a recent study, Solowska et al. (2008) showed that over-expression of a truncated form of long isoform of spastin depicts deleterious effects to axonal growth and inhibits fast axonal transport, which is consistent with a 'gain of function' mechanism.

However, to resolve the issue of 'gain/loss of function' further in vivo model systems including knockin and transgenic mouse models should be devised for various types of spastin disease causing mutations. These different pathomechanism models for mutant spastin mode of action need to be carefully resolved by experimental means; otherwise there will be repercussion on the likely success on any therapeutical approach devised for spastin-associated HSP.

\section{Acknowledgements}

The authors would like to thank $H$. Hühn and $K$. Neifer for excellent technical assistance and M. Mariappan and U. Winter for helping us with gel filtration chromotagraphy. We would also like to thank E. I. Rugarli for kindly providing us with spastin polyclonal antibody. NS thanks Department of Biotechnology (India), for the support. LSS is supported by a fellowship from Council of Scientific and Industrial Research (India). This work was funded partly by the Deutsche Forschungsgemeinschaft Grant (Germany) and Tom Wahlig Stiftung Grant (Germany) to AUM.

\section{Supplementary material}

The following supplementary material is available for this article: Table S1. Interaction environment on one oligomerisation surface. Table S2. Interaction environment on another oligomerisation surface.

Table S3. Classification of known set of disease associated HSP missense mutations in AAA domain into different structural category.

Fig. S1. Purification and enzymatic ATPase assay of recombinant spastin.

Fig. S2. Alignment used for modeling AAA ATPase domain of spastin.

This material is available as part of the online article from: http:// www.blackwell-synergy.com/doi/abs/10.1111/j.1471-4159.2008. 05414.x (This link will take you to the article abstract).

Please note: Blackwell Publishing is not responsible for the content or functionality of any supplementary materials supplied by the authors. Any queries (other than missing material) should be directed to the corresponding author for the article.

\section{Statement of competing interests}

The authors declare that they have no competing interests.

\section{References}

Akoev V., Gogol E. P., Barnett M. E. and Zolkiewski M. (2004) Nucleotide-induced switch in oligomerization of the AAA+ ATPase ClpB. Protein Sci. 13, 567-574.

Altschul S. F., Madden T. L., Schaffer A. A., Zhang J., Zhang Z., Miller W. and Lipman D. J. (1997) Gapped BLAST and PSI-BLAST: a new generation of protein database search programs. Nucleic Acids Res. 25, 3389-3402.

Babst M., Wendland B., Estepa E. J. and Emr S. D. (1998) The Vps4p AAA ATPase regulates membrane association of a Vps protein complex required for normal endosome function. EMBO J. 17, 2982-2993.

Berman H. M., Westbrook J., Feng Z., Gilliland G., Bhat T. N., Weissig H., Shindyalov I. N. and Bourne P. E. (2000) The protein data bank. Nucleic Acids Res. 28, 235-242.

Ciccarelli F. D., Proukakis C., Patel H., Cross H., Azam S., Patton M. A., Bork P. and Crosby A. H. (2003) The identification of a conserved domain in both spartin and spastin, mutated in hereditary spastic paraplegia. Genomics 81, 437441.

Claudiani P., Riano E., Errico A., Andolfi G. and Rugarli E. I. (2005) Spastin subcellular localization is regulated through usage of different translation start sites and active export from the nucleus. Exp. Cell Res. 309, 358-369.

Confalonieri F. and Duguet M. (1995) A 200-amino acid ATPase module in search of a basic function. Bioessays 17, 639-650.

Dreveny I., Kondo H., Uchiyama K., Shaw A., Zhang X. and Freemont P. S. (2004) Structural basis of the interaction between the AAA ATPase p97/VCP and its adaptor protein p47. EMBO J. 23, 10301039 .

Errico A., Ballabio A. and Rugarli E. I. (2002) Spastin, the protein mutated in autosomal dominant hereditary spastic paraplegia, is involved in microtubule dynamics. Hum. Mol. Genet. 11, 153 163.

Errico A., Claudiani P., D'Addio M. and Rugarli E. I. (2004) Spastin interacts with the centrosomal protein NA14, and is enriched in the spindle pole, the midbody and the distal axon. Hum. Mol. Genet. 13, 2121-2132.

Evans K. J., Gomes E. R., Reisenweber S. M., Gundersen G. G. and Lauring B. P. (2005) Linking axonal degeneration to microtubule remodeling by spastin-mediated microtubule severing. J. Cell Biol. 168, 599-606.

Evans K., Keller C., Pavur K., Glasgow K., Conn B. and Lauring B. (2006) Interaction of two hereditary spastic paraplegia gene products, spastin and atlastin, suggests a common pathway for axonal maintenance. Proc. Natl Acad. Sci. USA 103, 10666-10671.

Fink J. K. (2003) The hereditary spastic paraplegias: nine genes and counting. Arch. Neurol. 60, 1045-1049.

Fonknechten N., Mavel D., Byrne P. et al. (2000) Spectrum of SPG4 mutations in autosomal dominant spastic paraplegia. Hum. Mol. Genet. 9, 637-644.

Frickey T. and Lupas A. N. (2004) Phylogenetic analysis of AAA proteins. J. Struct. Biol. 146, 2-10.

Goloubinoff P., Mogk A., Zvi A. P., Tomoyasu T. and Bukau B. (1999) Sequential mechanism of solubilization and refolding of stable protein aggregates by a bichaperone network. Proc. Natl Acad. Sci. USA 96, 13732-13737.

Hanson P. I. and Whiteheart S. W. (2005) AAA+ proteins: have engine, will work. Nat. Rev. Mol. Cell Biol. 6, 519-529. 
Harding A. E. (1983) Classification of the hereditary ataxias and paraplegias. Lancet 1, 1151-1155.

Hartman J. J. and Vale R. D. (1999) Microtubule disassembly by $\Lambda$ TPdependent oligomerization of the AAA enzyme katanin. Science 286, 782-785.

Hazan J., Fonknechten N., Mavel D. et al. (1999) Spastin, a new AAA protein, is altered in the most frequent form of autosomal dominant spastic paraplegia. Nat. Genet. 23, 296-303.

Ki C. S., Lee W. Y., Han D. H. et al. (2002) A novel missense mutation (I344K) in the SPG4gene in a Korean family with autosomaldominant hereditary spastic paraplegia. J. Hum. Genet. 47, 473-477.

Lindsey J. C., Lusher M. E., McDermott C. J., White K. D., Reid E., Rubinsztein D. C., Bashir R., Hazan J., Shaw P. J. and Bushby K. M. (2000) Mutation analysis of the spastin gene (SPG4) in patients with hereditary spastic paraparesis. J. Med. Genet. 37, 759-765.

Luthy R., Bowie J. U. and Eisenberg D. (1992) Assessment of protein models with three-dimensional profiles. Nature 356, 83-85.

Mannan A. U., Boehm J., Sauter S. M., Rauber A., Byrne P. C., Neesen J. and Engel W. (2006a) Spastin, the most commonly mutated protein in hereditary spastic paraplegia interacts with reticulon 1 an endoplasmic reticulum protein. Neurogenetics 7, 93-103.

Mannan A. U., Krawen P., Sauter S. M., Boehm J., Chronowska A., Paulus W., Neesen J. and Engel W. (2006b) ZFYVE27 (SPG33), a novel spastin-binding protein, is mutated in hereditary spastic paraplegia. Am. J. Hum. Genet. 79, 351-357.

McDermott C. J., Grierson A. J., Wood J. D., Bingley M., Wharton S. B., Bushby K. M. and Shaw P. J. (2003) Hereditary spastic paraparesis: disrupted intracellular transport associated with spastin mutation. Ann. Neurol. 54, 748-759.

McNally F. J. and Vale R. D. (1993) Identification of katanin, an ATPase that severs and disassembles stable microtubules. Cell 75, 419 429.

Motohashi K., Watanabe Y., Yohda M. and Yoshida M. (1999) Heatinactivated proteins are rescued by the DnaK.J-GrpE set and ClpB chaperones. Proc. Natl Acad. Sci. USA 96, 7184-7189.

Patel S. and Latterich M. (1998) The AAA team: related ATPases with diverse functions. Trends Cell Biol. 8, 65-71.

Patel H., Cross H., Proukakis C., Hershberger R., Bork P., Ciccarell F. D., Patton M. A., McKusick V. A. and Crosby A. H. (2002 SPG20 is mutated in Troyer syndrome, an hereditary spastic paraplegia. Nat. Genet. 31, 347-348.

Reid E., Connell J., Edwards T. L., Duley S., Brown S. E. and Sanderson C. M. (2005) The hereditary spastic paraplegia protein spastin interacts with the ESCRT-III complex-associated endosomal protein CHMP1B. Hum. Mol. Genet. 14, 19-38.

Roll-Mecak A. and Vale R. D. (2005) The Drosophila homologue of the hereditary spastic paraplegia protein, spastin, severs and disassembles microtubules. Curr. Biol. 15, 650-655.

Roll-Mecak A. and Vale R. D. (2008) Structural basis of microtubule severing by the hereditary spastic paraplegia protein spastin. Nature 451, 363-367.

Sali A. (1995) Comparative protein modeling by satisfaction of spatial restraints. Mol. Med. Today 1, 270-277.
Salinas S., Carazo-Salas R. E., Proukakis C., Cooper J. M., Weston A. E., Schiavo G. and Warner T. T. (2005) Human spastin has multiple microtubule-related functions. J. Neurochem. 95, 1411 1420.

Sanderson C. M., Connell J. W., Edwards T. L., Bright N. A., Duley S., Thompson A., Luzio J. P. and Reid E. (2006) Spastin and atlastin, two proteins mutated in autosomal-dominant hereditary spastic paraplegia, are binding partners. Hum. Mol. Genet. 15, 307-318.

Scott A., Chung H. Y., Gonciarz-Swiatek M. et al. (2005) Structural and mechanistic studies of VPS4 proteins. EMBO J. 24, 3658 3669 .

Sherwood N. T., Sun Q., Xue M., Zhang B. and Zinn K. (2004) Drosophila spastin regulates synaptic microtubule networks and is required for normal motor function. PLoS Biol. 2, e429.

Sobolev V., Sorokine A., Prilusky J., Abola E. E. and Edelman M. (1999) Automated analysis of interatomic contacts in proteins. Bioinformatics 15, 327-332.

Solowska J. M., Morfini G., Falnikar A., Himes B. T., Brady S. T., Huang D. and Baas P. W. (2008) Quantitative and functional analyses of spastin in the nervous system: implications for hereditary spastic paraplegia. J. Neurosci. 28, 2147-2157.

Tarrade A., Fassier C., Courageot S. et al. (2006) A mutation of spastin is responsible for swellings and impairment of transport in a region of axon characterized by changes in microtubule composition. Hum. Mol. Genet. 15, 3544-3558.

Trotta N., Orso G., Rossetto M. G., Daga A. and Broadie K. (2004) The hereditary spastic paraplegia gene, spastin, regulates microtubule stability to modulate synaptic structure and function. Curr. Biol. 14, 1135-1147.

Wang Q., Song C., Yang X. and Li C. C. (2003) D1 ring is stable and nucleotide-independent, whereas D2 ring undergoes major conformational changes during the ATPase cycle of p97-VCP. J. Biol. Chem. 278, 32784-32793.

White S. R., Evans K. J., Lary J., Cole J. L. and Lauring B. (2007) Recognition of C-terminal amino acids in tubulin by pore loops in spastin is important for microtubule severing. J. Cell Biol. 176, 995-1005.

Wood J. D., Landers J. A., Bingley M., McDermott C. J., ThomasMcArthur V., Gleadall L. J., Shaw P. J. and Cunliffe V. T. (2006) The microtubule-severing protein spastin is essential for axon outgrowth in the zebrafish embryo. Hum. Mol. Genet. 15, 2763 2771.

Yoshimori T., Yamagata F., Yamamoto A., Mizushima N., Kabeya Y., Nara A., Miwako I., Ohashi M., Ohsumi M. and Ohsumi Y. (2000) The mouse SKD1, a homologue of yeast Vps4p, is required for normal endosomal trafficking and morphology in mammalian cells. Mol. Biol. Cell 11, 747-763.

Yu W., Solowska J. M., Qiang L., Karabay A., Baird D. and Baas P. W (2005) Regulation of microtubule severing by katanin subunits during neuronal development. J. Neurosci. 25, 5573-5583.

Zolkiewski M. (1999) ClpB cooperates with DnaK, DnaJ, and GrpE in suppressing protein aggregation. A novel multi-chaperone system from Escherichia coli. J. Biol. Chem. 274, 28083-28086. 


\subsection{Compound heterozygosity in SPG4 gene causes hereditary spastic paraplegia.}

In this part of the thesis, the effect of threshold level of functional spastin was elucidated by characterization of an autosomal dominant hereditary spastic paraplegia (AD-HSP) family with compound heterozygous mutations. The presence of SPG4 compound heterozygous sequence variants c.131C $>\mathrm{T}$ and c.1687G $>\mathrm{A}$ in the index patient causes a severe form of AD-HSP. The sequence variant c.131C $>$ T (p.S44L) is a polymorphism and is known to act as a modifier for HSP. RT-PCR and QUASEP techniques demonstrated that the novel c. $1687 \mathrm{G}>\mathrm{A}$ mutation is a leaky or hypomorphic splice site mutation. At protein level, c. $1687 \mathrm{G}>\mathrm{A}$ leads to E563K amino acid change. Furthermore, in silico analysis predicted that E563K amino acid change can possibly damage the function of spastin. Our data strengthens the 'threshold effect model' for spastin, where the level of functional spastin higher than $50 \%$ might lie above that of critical threshold level for the appearance of HSP.

Pantakani DVK, Zechner U, Arygriou L, Pauli S, Sauter SM and Mannan AU.

Status: Published in Clinical Genetics (Impact factor 3.181), Volume 73 (2008), pp 268272.

\section{Author contributions to the work:}

1. Pantakani DVK: Isolation of total RNA from patient samples and performing RT-PCR analysis and sequencing. Analysis using in silico methods. Involved in the manuscript preparation.

2. Zechner U: Performed the QUASEP analysis.

3. Arygriou L: Patients data collection and mutational analysis.

4. Pauli S: Patients data collection and mutational analysis.

5. Sauter SM: Patients data collection and mutational analysis.

6. Mannan AU: Conceived and designed the experiments. Interpretated the data and prepared the manuscript. 


\section{Short Report}

\section{Compound heterozygosity in the SPG4 gene causes hereditary spastic paraplegia}

Pantakani DVK, Zechner U, Arygriou L, Pauli S, Sauter SM, Mannan AU. Compound heterozygosity in the SPG4 gene causes hereditary spastic paraplegia.

Clin Genet 2008: 73: 268-272. (C) Blackwell Munksgaard, 2008

The SPG4 gene is frequently mutated in autosomal dominant form of hereditary spastic paraplegia (HSP). We report that the compound heterozygous sequence variants $\mathrm{S} 44 \mathrm{~L}$, a known polymorphism, and c. $1687 \mathrm{G}>\mathrm{A}$, a novel mutation in SPG4 cause a severe form of HSP in a patient. The family members carrying solely c. $1687 \mathrm{G}>\mathrm{A}$ mutation are asymptomatic for HSP. The reverse transcriptase-polymerase chain reaction (RT-PCR) analysis revealed that the c. $1687 \mathrm{G}>\mathrm{A}$ mutation is a splice site mutation and causes skipping of the exon 15 of spastin. Furthermore, quantification of RT-PCR products by sequencing and quantification of allele-specific expression by pyrosequencing assay revealed that c. $1687 \mathrm{G}>\mathrm{A}$ is a leaky or hypomorphic splice site mutation. At the protein level, c. $1687 \mathrm{G}>$ A mutation in $S P G 4$ leads to E563K substitution. In ex vivo study, about $10 \%$ of cells expressing E563K mutant spastin showed filamentous expression pattern, suggesting a hypomorphic effect at the protein level. Collectively, our results suggest that S44L in association with c.1687G $>$ A (E563K) drops the functional level of spastin below a threshold limit sufficient to manifest HSP.

\section{DVK Pantakani ${ }^{\mathbf{a}, *}$, U Zechner ${ }^{\mathrm{b}, *}$, L Arygriou ${ }^{a}$, S Pauli ${ }^{a}$, SM Sauter ${ }^{a}$ and AU Mannan ${ }^{a}$}

anstitute of Human Genetics, University of Goettingen, Goettingen, Germany, and bInstitute of Human Genetics, Johannes Gutenberg University, Mainz, Germany

*These authors equally contributed.

Key words: HSP - hypomorphic mutation - SPG4 - splice site

Corresponding author: Dr Ashraf U. Mannan, Institute of Human Genetics, University of Goettingen, HeinrichDueker-Weg 12, D-37073 Goettingen, Germany.

Tel.: +49551 397522;

fax: +49551 399303;

e-mail: amannan@gwdg.de

Received 8 August 2007, revised and accepted for publication 2 November 2007
Hereditary spastic paraplegias (HSPs) are a group of neurodegenerative disorders, which are clinically characterized by progressive spastic paralysis of the legs, usually caused by length-dependent distal degeneration of the corticospinal tract axons (1). The HSPs are genetically heterogeneous, and till now, 36 loci have been identified. However, mutations in the SPG4 gene (2) account for up to $40 \%$ of autosomal dominant $\operatorname{HSP}(3,4)$.

More than 150 different mutations have been identified in all exons of the SPG4 gene, and all types of DNA alterations are observed, including missense, nonsense, splice site mutations and small insertions/deletions $(3,4)$. This wide spectrum of mutations suggests that the pathogenic mechanism is haploinsufficiency $(3,4)$.

Interestingly, almost all missense mutations in the SPG4 gene, besides few exceptions, are located in the C-terminal AAA domain (2-4). Furthermore, a recent study suggests that some of these missense mutations might exert a dominant negative effect on the molecular function of spastin by disrupting the ATPase activity of the
AAA cassette, leading to aberration in microtubule dynamics (5). Another set of studies suggest that the sequence variants S44L and P45Q might inflict a hypomorphic activity to spastin and in association with another disease-causing missense mutation on AAA domain in the other allele could significantly increase the severity of the HSP symptoms such as a striking decrease in the age of onset $(4,6-8)$. Collectively, it appears that there is an association between different types or sites of mutations in SPG4 and severity of the manifested HSP phenotype. However, the correlation between the variable expressivity of HSP and the different types/sites of mutations in spastin is not yet explicit.

\section{Results}

Identification of a novel mutation in the SPG4 gene in a German family

We performed a mutational screen in the SPG4 gene in the genomic DNA of an index patient 
Compound heterozygosity in SPG4 gene

(Fig. 1, II-1) who shows a severe form of pure HSP with early onset of symptoms (Supplementary online information: Materials and Methods). We identified compound heterozygosity for sequence variants p.S44L (c.131C $>$ T), a known polymorphism $(4,6-8)$, and p.E563K (c. $1687 \mathrm{G}>\mathrm{A})$, a novel mutation in SPG4 gene in the index patient. Sequencing of the SPG4 gene in the genomic DNA of both parents and two brothers of the index patient (Fig. 1) revealed that the mother is heterozygous for the S44L polymorphism (Fig. 1, I-2), and the father is heterozygous for c.1687G $>$ A (Fig. 1, I-1). Moreover, both brothers (II-2 and II-3) of the index patient only inherited c. $1687 \mathrm{G}>\mathrm{A}$ from the father (Fig. 1). All family members except the index patient are asymptomatic for HSP. The c. $1687 \mathrm{G}>\mathrm{A}$ sequence variant in SPG4 gene can be classified as a novel mutation, as we did not detect this sequence variant in the genomic DNA of 100 control individuals. Thus, the possibility of the c. $1687 \mathrm{G}>\mathrm{A}$ sequence variant being a rare polymorphism is ruled out.

Sequence variant c. $1687 G>A$ in SPG4 gene is a splice site mutation

The identified mutation c. $1687 \mathrm{G}>\mathrm{A}$ is positioned at the last base of the exon 15, which is just followed by the splice donor site. In silico analysis using a splice site prediction program (http://www.fruitfly.org/seq_tools/splice.html) showed that c. $1687 \mathrm{G}>\mathrm{A}$ variation in SPG4 gene diminishes the potential of the splice donor site of exon 15 from $99-76 \%$. Furthermore, reverse transcriptase-polymerase chain reaction (RTPCR) amplification of spastin transcript (spanning exon 12 till exon 17) from the RNA of the index patient (II-1) and her father (I-1) revealed a smaller and weaker RT-PCR product of size approximately $181 \mathrm{bp}$ in addition to the fulllength product of size approximately $252 \mathrm{bp}$ (Fig. 2a). Sequencing of this smaller RT-PCR product confirmed that the resultant transcript lacked the exon 15 of spastin (Fig. 2b).

\section{Quantitative analysis of SPG4 transcript variants}

To quantify the relative transcript levels arising from c. $1687 \mathrm{G}$ allele over A allele, we amplified this region by RT-PCR. We cloned the RT-PCR product and sequenced approximately 100 plasmid samples. A preferential expression of the c. $1687 \mathrm{G}$ allele over the A allele was observed in the index patient with a ratio of $66 \%(\mathrm{G})$ to $34 \%$ (A), whereas her father showed $\mathrm{G}(58 \%)$ to A $(42 \%)$ level of expression (data not shown). Next, we used the quantification of allele-specific expression by pyrosequencing (QUASEP) to accurately determine the relative amount of the spastin transcripts from $\mathrm{C}$ vs $\mathrm{T}$ allele (reverse sequence). The results from QUASEP experiments indicate a preferential expression of the $\mathrm{C}$ allele of the spastin in the index patient and her father (Fig. 3a). This preferential expression seems to be more pronounced in the affected patient, who showed $63 \%$ of $\mathrm{C}$ allele as compared with her father, who showed $56 \%$ expression of the $\mathrm{C}$ allele
Fig. 1. Pedigree of a German hereditary spastic paraplegia (HSP) family showing segregation of c.131C $>\mathrm{T}(\mathrm{S} 44 \mathrm{~L})$ and c. $1687 \mathrm{G}>\mathrm{A}(\mathrm{E} 563 \mathrm{~K})$ sequence variants of $S P G 4$ in the family members. The index patient (II-1) is compound heterozygous and shows a severe phenotype. The other family members are only heterozygous for either of the sequence variants and are asymptomatic for HSP. The age of the respective individuals is provided in the pedigree in Arabic numerical. Sequence data for probands (II-2 and II-3) were not performed in our institute and were unavailable to us.

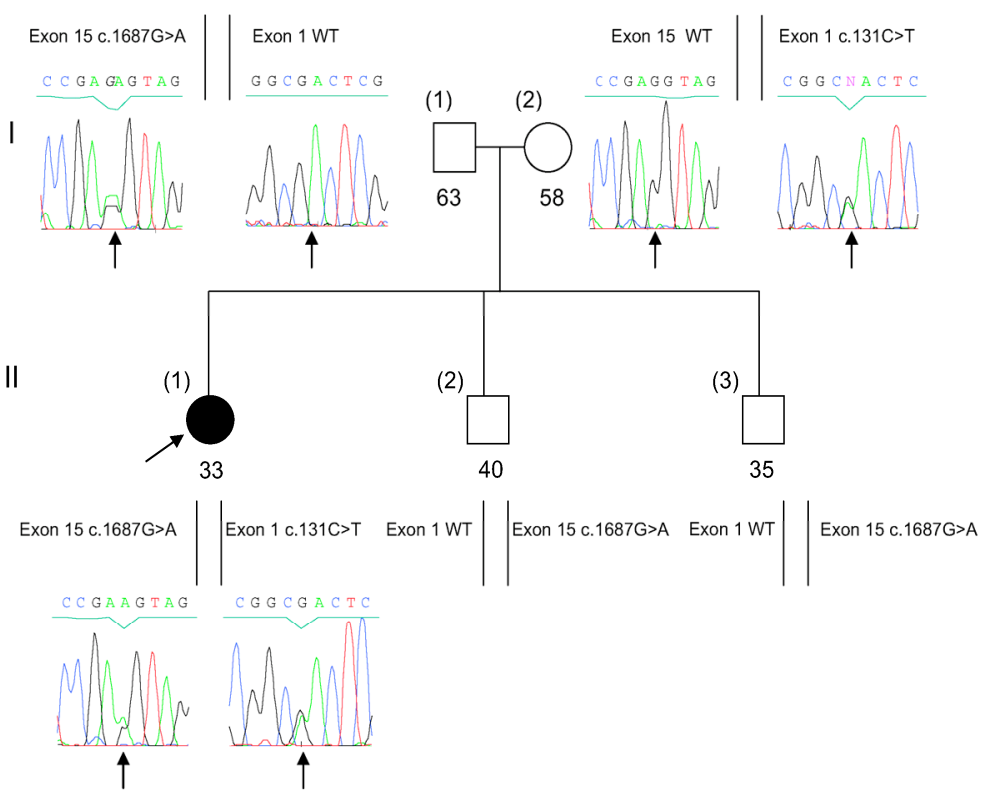




\section{Pantakani et al.}

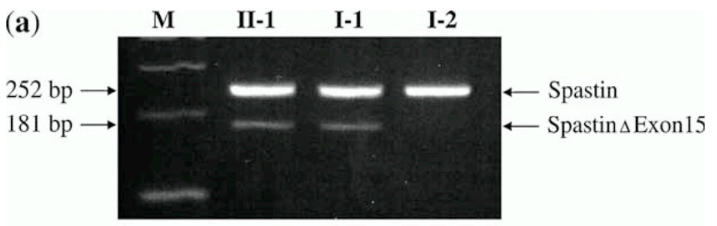

(b)

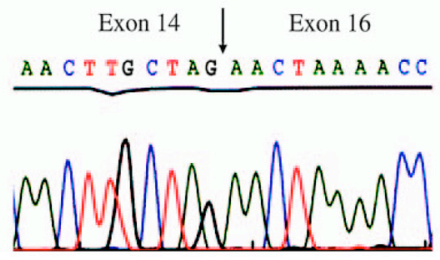

Fig. 2. The skipping of exon 15 of spastin because of c. $1687 \mathrm{G}>$ A mutation. The reverse transcriptase-polymerase chain reaction (RT-PCR) amplification of exon 12 till exon 17 of the spastin from RNA of the index patient (II-1), her father (I-1) and mother (I-2). Two bands of sizes $181 \mathrm{bp}$ and 252 bp could be seen in the RNA samples of the patients (II-1 and I-1) carrying c. $1687 \mathrm{G}>\mathrm{A}$ mutation. However, only one band of size $252 \mathrm{bp}$ was detected in the patient, I-2, who is non-carrier for the mutation (a). M, 100-bp molecular weight marker. Sequencing of the RT-PCR product of size $181 \mathrm{bp}$ revealed skipping of the exon 15 of spastin because of the c. $1687 \mathrm{G}>$ A splice site mutation (b).
(Fig. 3b,c). The mother (wild type) and the control individuals showed the expected expression levels of the $\mathrm{C}$ allele between $96 \%$ and $100 \%$ (Fig. 3b,c).

\section{In silico analysis of spastin E563K substitution}

The c. $1687 \mathrm{G}>\mathrm{A}$ mutation leads to amino acid substitution E563K at the protein level. To determine the effect of E563K substitution on the function of spastin, we used SIFT Version 2 program (Sorting of Intolerant From Tolerant; http://blocks.fhcrc.org/sift/SIFT.html), which considered sequences of 63 closely related spastin paralogues and orthologues in its calculation. The SIFT analysis indicated that the E563K substitution in spastin is intolerable with threshold value of 0.03. Similarly, POLYPHEN (Polymorphism Phenotyping; http://genetics.bwh.harvard. $\mathrm{edu} / \mathrm{pph} /$ ) prediction data also suggest that the spastin E563K substitution can possibly be damaging to the function of spastin with positionspecific independent counts value of 1.533 on the basis of alignment.

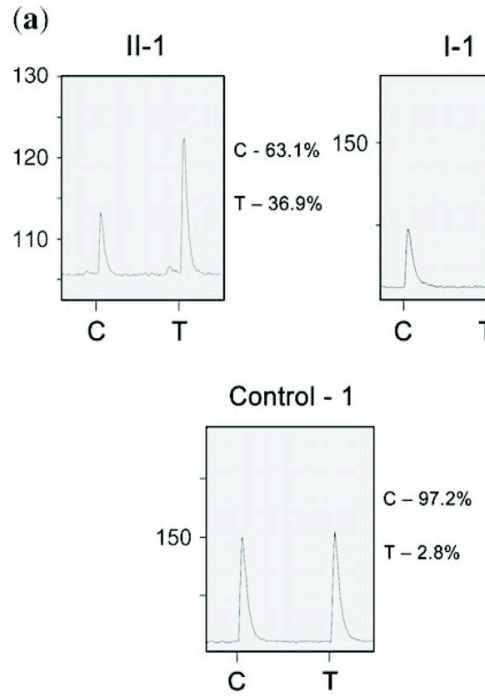

(b)

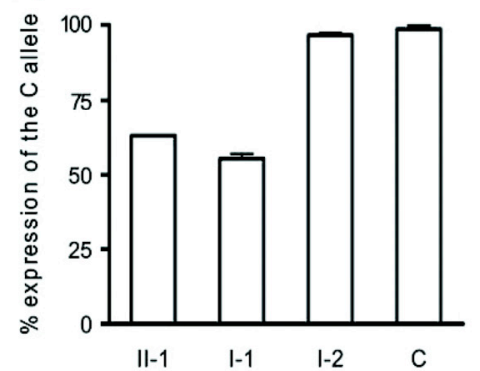

l-1

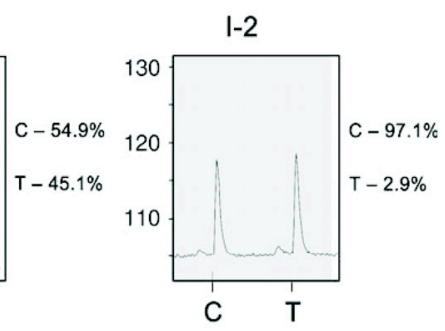

Control - 2

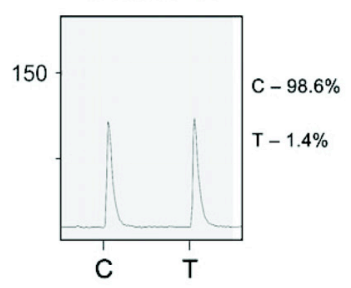

(c)

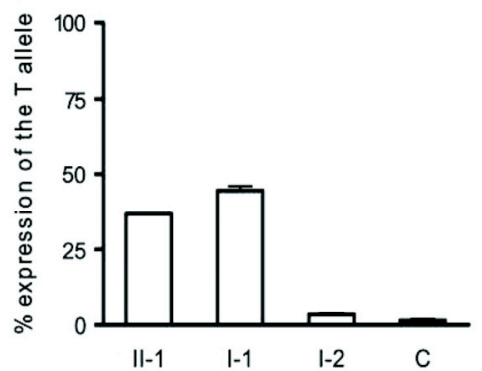

Fig. 3. Absolute quantification of c. $1687 \mathrm{G} v s$ A allele transcripts by quantification of allele-specific expression by pyrosequencing (QUASEP) assays. The pyrosequencing pyrograms of cDNA derived from the hereditary spastic paraplegia family members (I-1, I-2 and II-1) and control unaffected individuals (control-1 and control-2) are shown. The pyrogram values indicate a preferential expression of the SPG4 C allele (reverse sequence) in II-1 and I-1 (a). Representation of QUASEP results in histogram forms (b and $\mathbf{c}$ ). The expression of the mutated $\mathrm{T}$ allele (reverse sequence) at position 1687 can only be quantified by deducting the basal peak value of the $\mathrm{T}$ base at position 1686 from the observed $T$ peak in the pyrogram. Minimal T peak values of $1.4-2.8 \%$ at position 1687 in control individuals are because of background noise, which reproducibly occurred to the same extent within runs and most probably resulted from sequence-dependent nucleotide misincorporations or small impurities in the deoxyribonucleotide triphosphate reagent mixture. C stands for pooled control samples. 


\section{Compound heterozygosity in SPG4 gene}

Aberrant intracellular distribution of E563K spastin in cell

We introduced E563K mutation in a previously generated c-Myc-spastin construct (9) and transfected the HeLa cells. Evaluation of the cells expressing c-Myc-E563K-spastin demonstrated that a small fraction of cells showed an atypical filamentous expression pattern (Fig. S1b, Supplementary material online), beside the typical punctate expression of spastin around the perinuclear region (Fig. S1a, Supplementary material online). Quantitative analysis of more than 100 cells expressing c-Myc-E563K-spastin revealed that about $10 \%$ of all cells showed filamentous distribution (Fig. S1c, Supplementary material online).

\section{Discussion}

In the present study, we report that compound heterozygous sequence variants [c.131C $>$ T (S44L) and c.1687G $>$ A (E563K)] occurring on different alleles of the SPG4 gene cause a severe form of spastic paraplegia in a patient. The S44L polymorphism acts as a modifier of HSP phenotype when it occurs in association with another disease-causing spastin mutation $(4,6-8)$. In our case study, the mutation c. $1687 \mathrm{G}>\mathrm{A}$ (alone) appears to be nonpathogenic as family members carrying solely the c. $1687 \mathrm{G}>\mathrm{A}$ allele are not manifesting any symptoms of HSP. However, the possibility of a reduced penetrance of c. $1687 \mathrm{G}>\mathrm{A}$ mutation cannot be completely ruled out.

The splice site prediction program indicated that c. $1687 \mathrm{G}>\mathrm{A}$ variation in the SPG4 gene diminishes the potential of splice donor site from $99 \%$ to $76 \%$. The RT-PCR analysis confirmed that the c. $1687 \mathrm{G}>\mathrm{A}$ mutation is indeed a splice site mutation and causes skipping of the exon 15 of spastin. Moreover, the observation of a weaker intensity of band corresponding to the smaller variant of spastin in the RT-PCR amplification as compared with endogenous spastin transcript suggests that c. $1687 \mathrm{G}>\mathrm{A}$ might be a leaky splice site mutation. The quantification of c.1687G vs A allelic transcript variants of the spastin by sequencing of the RT-PCR products as well as by QUASEP assay from the index patient and her father confirmed that c. $1687 \mathrm{G}>\mathrm{A}$ is a leaky or hypomorphic splice mutation. The action of c. $1687 \mathrm{G}>\mathrm{A}$ mutation as a hypomorphic splice mutation could explain the lack of HSP symptoms in the family members carrying only c. $1687 \mathrm{G}>\mathrm{A}$ mutation in a heterozygous state.
At the protein level, c. $1687 \mathrm{G}>\mathrm{A}$ mutation leads to E563K substitution. In silico analyses using both SIFT and POLYPHEN programs revealed that E563K substitution can possibly be damaging the function of spastin. To determine the effect of this mutation on spastin protein, we studied the intracellular distribution of E563Kspastin protein in HeLa cells. About $10 \%$ of cells expressing mutant spastin showed filamentous expression pattern, suggesting a hypomorphic effect on the protein.

The level of functional spastin might be critical for appearance of the HSP. Below a threshold limit, the disease will be manifested and a level of functional spastin higher than $50 \%$ might lie above that of critical threshold level. Therefore, family members solely carrying the c. $1687 \mathrm{G}>\mathrm{A}$ mutation appear normal. However, the index patient besides carrying c. $1687 \mathrm{G}>\mathrm{A}$ mutation on one allele also carries the S44L polymorphism on the other allele, therefore, both variants together lead to hypomorphic spastin (reduced amount and activity), which plunges the functional level of spastin below the threshold amount and generate HSP symptoms.

Our findings further strengthen the threshold effect model' for spastin $(10,11)$ and suggest that there is a direct correlation between functional level of spastin and symptom severity in the spastin-linked HSP.

\section{Supplementary material}

Fig. S1. Intracellular distribution of wild-type and mutant spastin in HeLa cells.

Supplementary material is available as part of the online article at http://www.blackwell-synergy.com/

\section{Acknowledgements}

The authors would like to thank the HSP family for their participation in this study and B. Brandt and D. Weise for excellent technical assistance. This work was funded partly by the Deutsche Forschungsgemeinschaft Grant and Tom Wahlig Stiftung Grant to A. U. M.

\section{References}

1. Fink JK. The hereditary spastic paraplegias: nine genes and counting. Arch Neurol 2003: 60: 1045-1049.

2. Hazan J, Fonknechten N, Mavel D et al. Spastin, a new AAA protein, is altered in the most frequent form of autosomal dominant spastic paraplegia. Nat Genet 1999: 23: 296-303

3. Fonknechten N, Mavel D, Byrne P et al. Spectrum of SPG4 mutations in autosomal dominant spastic paraplegia. Hum Mol Genet 2000: 9: 637-644. 


\section{Pantakani et al.}

4. Lindsey JC, Lusher ME, McDermott CJ et al. Mutation analysis of the spastin gene (SPG4) in patients with hereditary spastic paraparesis. J Med Genet 2000: 37: 759-765.

5. Errico A, Ballabio A, Rugarli EI. Spastin, the protein mutated in autosomal dominant hereditary spastic paraplegia, is involved in microtubule dynamics. Hum Mol Genet 2002: 11: 153-163.

6. Svenson IK, Kloos MT, Gaskell PC et al. Intragenic modifiers of hereditary spastic paraplegia due to spastin gene mutations. Neurogenetics 2004: 5: 157-164.

7. Chinnery PF, Keers SM, Holden MJ et al. Infantile hereditary spastic paraparesis due to codominant mutations in the spastin gene. Neurology 2004: 63: 710-712.
8. McDermott CJ, Burness CE, Kirby J et al. UK and Irish HSP Consortium. Clinical features of hereditary spastic paraplegia due to spastin mutation. Neurology 2006: 67 (1): 45-51.

9. Mannan AU, Boehm J, Sauter SM et al. Spastin, the most commonly mutated protein in hereditary spastic paraplegia interacts with Reticulon 1 an endoplasmic reticulum protein. Neurogenetics 2006: 7: 93-103.

10. Svenson IK, Ashley-Koch AE, Gaskell PC et al. Identification and expression analysis of spastin gene mutations in hereditary spastic paraplegia. Am J Hum Genet 2001: 68: 1077-1085.

11. Svenson IK, Ashley-Koch AE, Pericak-Vance MA et al. A second leaky splice-site mutation in the spastin gene. Am J Hum Genet 2001: 69: 1407-1409. 


\subsection{Expansion of mutation spectrum, determination of mutation cluster regions and predictive structural classification of SPAST mutations in hereditary spastic paraplegia.}

Identification of a large set of novel spastin (SPAST/SPG4) mutations in a cohort of hereditary spastic paraplegia (HSP) patients has been reported in this part of the thesis. By employing standard mutational screening methods and unique MLPA (multiplex ligationdependent probe amplification) technique, a total of 29 novel mutations were identified in the current study. Clustering of mutations was examined over the functional regions of spastin. Finally, the previously modeled spastin structure was used to categorize the newly identified mutations into various structural/functional groups. Moreover our data indicates that in the case of missense mutations, the onset of phenotype is earlier and suggests a tentative genotype-phenotype correlation.

Moneef Shoukier, Juergen Neesen, Simone M Sauter, Loukas Argyriou, Nadine Doerwald, DV Krishna Pantakani and Ashraf U. Mannan

Status: Published in European Journal of Human Genetics (Impact factor 4.003), Volume 17 (2009), pp 187-194.

\section{Author contributions to the work:}

1. Moneef Shoukier: Mutational screening of HSP patients and collection of data.

2. Jurgen Neesen: Mutational screening of HSP patients.

3. Simone M Sauter: Mutational screening of HSP patients.

4. Loukas Argyriou: Mutational screening of HSP patients.

5. Nadine Doerwald: Mutational screening of HSP patients.

6. DV Krishna Pantakani: Structural/functional categorization of newly identified mutations using modeled spastin structure. Involved in the manuscript preparation.

7. Ashraf U. Mannan: Conceived and designed the experiments. Interpretated the data and prepared the manuscript. 


\title{
Expansion of mutation spectrum, determination of mutation cluster regions and predictive structural classification of SPAST mutations in hereditary spastic paraplegia
}

\author{
Moneef Shoukier ${ }^{1,2}$, Juergen Neesen ${ }^{1,2,3}$, Simone M Sauter ${ }^{1}$, Loukas Argyriou ${ }^{1}$, \\ Nadine Doerwald ${ }^{1}$, DV Krishna Pantakani ${ }^{1}$ and Ashraf U Mannan*,1 \\ ${ }^{1}$ Institute of Human Genetics, University of Goettingen, Goettingen, Germany
}

The SPAST gene encoding for spastin plays a central role in the genetically heterogeneous group of diseases termed hereditary spastic paraplegia (HSP). In this study, we attempted to expand and refine the genetic and phenotypic characteristics of SPAST associated HSP by examining a large cohort of HSP patients/families. Screening of 200 unrelated HSP cases for mutations in the SPAST gene led to detection of 57 mutations $(28.5 \%)$, of which 47 were distinct and 29 were novel mutations. The distribution analysis of known SPAST mutations over the structural domains of spastin led to the identification of several regions where the mutations were clustered. Mainly, the clustering was observed in the AAA (ATPases associated with diverse cellular activities) domain; however, significant clustering was also observed in the MIT (microtubule interacting and trafficking), MTBD (microtubule-binding domain) and an N-terminal region (228-269 residues). Furthermore, we used a previously generated structural model of spastin as a framework to classify the missense mutations in the AAA domain from the HSP patients into different structural/functional groups. Our data also suggest a tentative genotype-phenotype correlation and indicate that the missense mutations could cause an earlier onset of the disease.

European Journal of Human Genetics (2009) 17, 187-194; doi:10.1038/ejhg.2008.147; published online 13 August 2008

Keywords: SPAST; spastin; HSP; mutation

Introduction

Hereditary spastic paraplegias (HSPs), also known as the Strümpell-Lorrain syndrome, are a group of neurodegenerative disorders caused by monogenic mutations. The universal clinical feature of the disease is progressive bilateral weakness and spasticity of the lower limbs. ${ }^{1}$

${ }^{*}$ Correspondence: Dr AU Mannan, Institute of Human Genetics, University of Goettingen, Heinrich-Dueker-Weg 12, D-37073, Goettingen, Germany. Tel: + 49551 397522; Fax + 49551 399303;

E-mail: amannan@gwdg.de

${ }^{2}$ These authors contributed equally to this work

${ }^{3}$ Current address: Department of Medical Genetics, Medical University of Vienna, Vienna, Austria

Received 15 January 2008; revised 9 July 2008; accepted 11 July 2008; published online 13 August 2008
Histopathological studies of HSP patients revealed selective degeneration of some of the longest axons. The degenerative process initially affects the distal ends of these axons and then proceeds proximally toward the cell body. ${ }^{2,3}$ On the basis of clinical features, HSP can be classified into two forms: the pure and the complex HSP. In pure HSP, spasticity occurs in relative isolation; however, when additional neurological or non-neurological symptoms are associated with spasticity then it is termed as complex HSP., ${ }^{4,5}$

HSPs are genetically heterogeneous and different modes of inheritance are reported, including autosomal dominant, recessive and X-linked HSP; within each inheritance group, there is further locus heterogeneity. Fifteen of the 


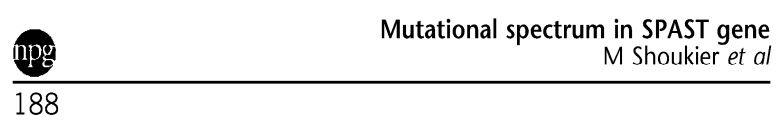

HSP genes have been identified. However, mutations in the SPAST gene (MIM 604277) encoding for spastin protein is the most common cause of $\mathrm{HSP}^{6}$ and accounts for $15-40 \%$ of all AD-HSP cases, depending upon the ethnic origin of the selected cohort of patients. ${ }^{6-12}$ The prevalence of SPAST mutations in sporadic cases and with uncertain family history is much lower (12-18\%). ${ }^{13,14}$

Spastin belongs to the AAA (ATPases associated with diverse cellular activities) family of proteins. ${ }^{15-17}$ The AAA proteins are proven or putative ATPases and they are characterized by a conserved C-terminal domain containing one or two AAA cassettes. ${ }^{15}$ Apart from the AAA domain, these proteins consist of various other domains, which interact with adapter proteins to generate the structural and functional diversity of the family. ${ }^{16,18}$

Screening for mutations in the SPAST gene by various groups has identified over 224 different mutations in most exons except for exon 4, which is alternatively spliced. The SPAST mutations are summarized in the Human Gene Mutation Database Professional release 7.1 (http:// www.biobase.de/hgmd/pro/start.php). Different types of DNA alterations are detected in the SPAST gene, including missense, nonsense, splice site mutations and insertions/ deletions. Recent studies used a SPAST-specific multiplex ligation-dependent probe amplification (MLPA) assay to demonstrate that a large proportion (18-20\%) of patients with unlinked HSP, in fact, carry large deletions in the SPAST gene. $^{19,20}$ These findings further broaden the spectrum of the SPAST mutations.

From a diagnostic point of view, unfortunately, there are no common mutations in SPAST, with most families having private mutations. Moreover, there are neither obvious genotype-phenotype correlations between different types of spastin mutations, nor any hot spot regions for SPAST mutations have been identified. Therefore, screening of the complete coding sequence of SPAST is necessary for the detection of mutations.

\section{Materials and methods \\ HSP cases/families}

Blood samples from 200 unrelated HSP individuals/ families, primarily from Germany were referred to Goettingen Molecular Genetics Service for diagnostic testing of the SPAST gene. Clinical data of these patients were obtained by clinical evaluations from the referring neurologist. All probands were selected on the basis of Harding's accepted criteria for the definition of the clinical status of HSP. ${ }^{21}$ For ascertaining, a patient as HSP case following clinical features were assessed/observed in the lower limbs (lower extremities) of the patient; gait disorder owing to spastic paraparesis, spastic hypertonia, positive Babinski sign, ankle clonus, hyperreflexia, reduced pallesthesia and paresis. In addition, for complex HSP, cognitive deficits and other neurological/non-neurological symptoms were also considered. The age at onset (AAO) was determined by the referring neurologist after clinical evaluation and consulting with the patient. If required, additional family members were also consulted to corroborate the AAO. In our HSP cohort, 99 cases showed familial inheritance, 46 sporadic cases and in 55 cases, we could not ascertain the mode of inheritance. In this cohort, there were 109 pure HSP, 23 complex HSP and 68 unknown cases. Informed consent was obtained from all probands.

\section{Mutation screening and detection}

Genomic DNA from peripheral blood leukocytes of patients was isolated using standard procedures. The 17 exons of the SPAST gene (accession no. NM_014946.3) and flanking intronic sequences were amplified by PCR. Primer sequences and PCR conditions are available on request. Purification of PCR products was performed using a PCR purification kit (Millipore). The purified PCR products were sequenced using both forward and reverse primers (which were used for the PCR amplification) using ET reaction kit (Amersham Biosciences) on a MegaBACE 500 sequencer (Amersham Biosciences). Nucleotide variations revealed by first sequencing reaction were verified by second independent PCR and sequencing reaction. All sequence variants reported here were checked in a panel of 50 healthy unrelated subjects recruited randomly from the German population.

\section{MLPA analysis}

MLPA was performed with $200 \mathrm{ng}$ of genomic DNA according to manufacturer's instructions using the P165 Salsa MLPA HSP probe set (MRC-Holland). Probe amplification products were run on an ABI 3130 DNA Analyzer using Liz600 size standard (Applied Biosystems). MLPA peak plots were visualized, normalized and the dosage ratios were calculated by using GeneMarker Software v1.51 (Soft Genetics LLC). Owing to variation in each assay performance, we used dosage ratio values of $\leq 0.7$ and $\geq 1.35$ as our boundaries for deletions and duplications, respectively.

\section{Detection of break points for small insertions and deletions}

To determine the precise breakpoints of small insertions and deletions in the SPAST gene, the PCR amplicons were cloned into pGEMT Easy vector (Promega) according to the manufacturer's instructions. Plasmid DNA from at least 10 independent bacterial colonies were sequenced in both directions using vector-specific primers (T7 and SP6) using standard protocol.

\section{Modeling of the AAA domain of spastin}

The AAA ATPase domain of spastin was modeled on the basis of the tertiary structures of two templates (PDB codes: 1xwi and 1s3s) using MODELLER Version 8.0 program. ${ }^{22}$ 
The generated models were energy-minimized using the Kollman united atom force field in SYBYL (Tripos Inc.) to ensure acceptable geometry and to relieve short contacts. The overall fit of the sequence to the template was checked using Verify 3D. ${ }^{22}$ The copies of modeled tertiary structures were assembled to form a hexameric quaternary assembly on the basis of the hexameric template (1s3s). This modeled quaternary structure was energy-minimized using SYBYL. ${ }^{22}$ The interfacial residues between the monomers were extracted using the Contacts of Structural Units program $^{22}$ and the crucial residues were short listed by manual inspection and used for further analysis.

\section{Labeling of mutated missense residues in the structural model of spastin}

We used a previously generated structural model of spastin, $^{22}$ for labeling of the missense mutations identified in the AAA domain in our HSP cohort. In brief, the tertiary structure of the ATPase domain of spastin was modeled on the basis of the tertiary structures of two templates (PDB codes: $1 \mathrm{xwi}$ and 1s3s) and the quaternary structure was modeled on the basis of the hexameric template (1s3s). The consensus sequence/motifs in the AAA domain of spastin were structurally classified as active site, pore loop, protomer interacting and other residues and labeled as colored ribbons in the modeled tertiary structure. The novel spastin missense mutations identified in this study were labeled as colored space-fill structures in tertiary and quaternary structures using RasWin Molecular Graphics Version 2.7.3. (http://www.rasmol.org/).

The models of spastin are deposited in Protein Model Data Base (http://mi.caspur.it/PMDB/), which can be downloaded as PDB files (PDB codes: PM0074982 and PM0074984). The amino-acid residues in the spastin model are numbered from 1-286 aa, which corresponds to 331616 aa of the full-length spastin isoform. To determine in which functional/structural group (active site, pore loop structure, protomer interface residues and other mutations) of spastin a novel sequence variant can be cataloged, the mutated residue can be labeled by using RasWin Molecular Graphics Version 2.7.3 program.

\section{Results}

Detection of SPAST mutations in the HSP cases by sequencing and MLPA analysis

A total of 200 DNA samples of unrelated HSP individuals/ families were first screened for mutations in the SPAST gene by sequencing all the 17 exons of the gene, which led to the identification of 51 (25.5\%) heterozygous mutations (Table 1). Next, to detect SPAST deletion, we performed MLPA analysis in the 149 HSP cases, which were identified as SPAST negative through sequence analysis. By MLPA analysis, we identified six (three of which represented novel mutations) additional large heterozygous deletions in the SPAST gene, which accounts for $4 \%$ of the 149 HSP cases (Table 1). In our screen, 47 different mutations were detected and 29 of these mutations were novel (Table 1). None of the 29 identified novel mutations in our study were found in the 100 control chromosomes. In the analyzed HSP cohort, the mutation rate in the SPAST gene in pure HSP was $29.4 \%(32 / 109)$, in complex HSP it was $21.7 \%(5 / 23)$ and in cases of unknown clinical course the proportion was $29.4 \%(20 / 68)$ (Table S1). Furthermore, in familial HSP we observed a mutation frequency of $36.4 \%$ $(36 / 99)$, in sporadic HSP, it was $6.5 \%(3 / 46)$ and in HSP cases with unknown inheritance pattern, we detected a mutation rate of $32.7 \%$ (18/55) ((Table S1), Note: Table S1S4 and Figure S1 are available as (online Supplementary Information)).

\section{Mutational spectrum and cluster regions in spastin} The distribution analysis of the 26 novel mutations (excluding the gross deletions) along the structural domains of spastin revealed that $22(85 \%)$ mutations are localized in the conserved AAA domain, two (8\%) mutations were in the MIT domain (Figure 1a). Owing to this skewed spectrum of mutations in the spastin, we included other spastin mutations reported in the database (http:// www.biobase.de/hgmd/pro/start.php) in our analysis. Interestingly, we could identify several regions within the structural architecture of spastin where mutations were clustered (Figure 1b). The primary region was in the AAA domain (342-599 aa), which accounts for $72.7 \%$ of identified mutations in spastin (Figure 1b and Table S2). Additional secondary cluster regions, were detected in the MIT domain (116-197 aa) with 7.2\% mutations, MTBD (microtubule-binding domain; $270-328$ aa) ${ }^{30}$ with $5.1 \%$ and an N-terminal region (228-269 residues) with $7.6 \%$ mutations (Figure $1 \mathrm{~b}$ and Table S2). Overall, these four regions account for $92.7 \%$ of the mutations reported to date in spastin.

\section{Functional/structural classification of the missense mutations}

Out of the 29 novel mutations, which we identified in our screen, 10 (38\%) are missense mutations, which are located in the AAA domain. We attempted to classify these 10 missense mutations identified in the AAA domain into different structural/functional groups based upon a previously generated structural model of spastin (PMBD id: PM0074982 and PM0074984). ${ }^{22}$ We could consign all these missense mutations in the three dimensional space of the spastin structure into four categories namely, active site, pore loop structure, protomer interface residues and other mutations (Table S3 and Figure S1A-K).

\section{S44L polymorphism in HSP}

Co-inheritance of a disease-causing mutation and the L44 allele causes an early onset of symptoms, indicating that 


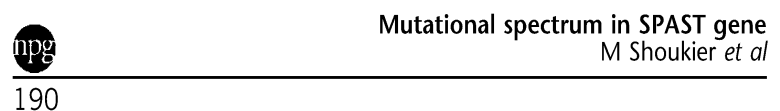

Table 1 List of mutations identified in the SPAST gene in our HSP cohort

\begin{tabular}{|c|c|c|c|c|c|c|c|c|c|}
\hline No. & Exon/Intron & Family no. & Type of mutation & $C D N A$ & Protein & $F S$ & $\begin{array}{l}\text { Onset of } \\
\text { phenotype }\end{array}$ & Pure/complex & $\begin{array}{c}\text { Referencel } \\
\text { novel }\end{array}$ \\
\hline 1 & Ex 1 & 24227 & Nonsense & c. $373 G>T$ & p.Glu125Term & $\mathrm{F}$ & $>35$ & Pure & 11 \\
\hline 2 & Ex 2 & 25942 & Nonsense & c. $499 \mathrm{C}>\mathrm{T}$ & p.GIn167Term & $S$ & $>35$ & Pure & Novel \\
\hline 3 & Ex 3 & 24283 & Insertion & C.549_550insT & p.Asn184Term & $\mathrm{F}$ & $<35$ & Pure & Novel \\
\hline 4 & Ex 5 & 21987 & Deletion & c. $692 \mathrm{delC}$ & $\begin{array}{l}\text { p.Ala231Valfs } \\
\text { Term239 }\end{array}$ & $?$ & $?$ & $?$ & Novel \\
\hline 5 & Ex 5 & 19583 & Deletion & c.839_840delAG & $\begin{array}{l}\text { p.GIn280Argfs } \\
\text { Term } 289\end{array}$ & $\mathrm{~F}$ & $<35$ & Pure & 12 \\
\hline 6 & Ex 5 & 28146 & Splice & c. $870 \mathrm{G}>\mathrm{A}$ & Unknown & ? & ? & $?$ & Novel \\
\hline 7 & Int 5 & 25961 & Splice & c. $870+1 \mathrm{G}>\mathrm{T}$ & Unknown & ? & ? & $?$ & Novel \\
\hline 8 & Ex 6 & 24295 & Insertion & c.981_982insAT & p.lle328llefs & $\dot{\mathrm{F}}$ & $<35$ & $\begin{array}{c}\text { Complicated } \\
\text { (cardio-myopathy) }\end{array}$ & Novel \\
\hline 9 & Ex 7 & 21937 & Missense & c. $1067 \mathrm{~A}>\mathrm{G}$ & p.Glu356Gly & $?$ & $<10$ & ? & 6 \\
\hline 10 & Ex 7 & 21935 & Missense & c. $1081 C>T$ & p.Pro361Ser & $\mathrm{F}$ & $<35$ & Pure & Novel \\
\hline 11 & Int 7 & 21971 & Splice/deletion & c.1099-3_1099-1delTAG & Unknown & $\mathrm{F}$ & $>35$ & Pure & Novel \\
\hline 12 & Ex 8 & 21977 & Deletion & c.1101_1103delGTT & p.Leu367del & $\mathrm{F}$ & $>35$ & Pure & Novel \\
\hline 13 & Ex 8 & 25902 & Deletion & c.1101_1103delGTT & p.Leu367del & $\mathrm{F}$ & $<10$ & Pure & Novel (2) \\
\hline 14 & Ex 8 & 24218 & Insertion & c.1115_1116insG & $\begin{array}{l}\text { p.Arg372Argfs } \\
\text { Term393 }\end{array}$ & $?$ & $?$ & $?$ & Novel \\
\hline 15 & Ex 8 & 25945 & Missense & c. $1121 C>G$ & p.Pro374Arg & $\mathrm{F}$ & $<10$ & $?$ & Novel \\
\hline 16 & Ex 8 & 25946 & Missense & c. $1154 \mathrm{G}>\mathrm{A}$ & p.Gly385Glu & $S$ & $>35$ & Pure & Novel \\
\hline 17 & Int 8 & 24292 & Splice & c. $1174-1 G>T$ & Unknown & $\mathrm{F}$ & $<35$ & Pure & Novel \\
\hline 18 & Ex 9 & 21938 & Missense & c. $1196 \mathrm{C}>\mathrm{T}$ & p.Ser399Leu & $\mathrm{F}$ & $?$ & $?$ & 23 \\
\hline 19 & Ex 9 & 24224 & Missense & c. $1196 C>T$ & p.Ser399Leu & ? & ? & $?$ & 23 \\
\hline 20 & Ex 9 & 19576 & Deletion & c.1202delC & $\begin{array}{l}\text { p.Ala401Gluf- } \\
\text { sTerm406 }\end{array}$ & ? & ? & ? & Novel \\
\hline 21 & Ex 9 & 24286 & Deletion & c.1215_1219delTATAA & $\begin{array}{l}\text { p.Asn405Lysf- } \\
\text { sTerm440 }\end{array}$ & $S$ & $>35$ & Pure & 8 \\
\hline 22 & Ex 9 & 24268 & Missense & c. $1216 A>G$ & p.lle406Val & $\mathrm{F}$ & $<35$ & Pure & 24 \\
\hline 23 & Ex 9 & 21901 & Deletion & c.1245delC & p.Tyr415Term & $S$ & $>35$ & Pure & Novel \\
\hline 24 & Int 9 & 24248 & Splice & c. $1245+1 G>T$ & Unknown & $\mathrm{F}$ & $<10$ & Pure & 25 \\
\hline 25 & Ex 10 & 24231 & Missense & c. $1250 \mathrm{G}>\mathrm{A}$ & p.Gly417Glu & $\mathrm{F}$ & $<35$ & $\begin{array}{l}\text { Complicated } \\
\text { (cognitive } \\
\text { impairment) }\end{array}$ & Novel \\
\hline 26 & Ex 10 & 19582 & Missense & c. $1280 \mathrm{~T}>\mathrm{G}$ & p.Phe427Cys & $\mathrm{F}$ & $>35$ & Pure & Novel \\
\hline 27 & Ex 10 & 24233 & Deletion & c.1281delT & $\begin{array}{l}\text { p.Phe427Leufs } \\
\text { Term437 }\end{array}$ & $?$ & $?$ & $?$ & 26 \\
\hline 28 & Ex 10 & 24212 & Nonsense & c. $1291 C>T$ & p.Arg431Term & $\mathrm{F}$ & $>35$ & Pure & 8 \\
\hline 29 & Ex 11 & 19593 & Missense & c. $1339 \mathrm{~T}>\mathrm{G}$ & p.Leu447Val & $\mathrm{F}$ & $>35$ & Pure & Novel \\
\hline 30 & Ex 11 & 21214 & Missense & c. $1378 . C>T$ & p.Arg460Cys & $F$ & $>35$ & $\begin{array}{c}\text { Complicated } \\
\text { (polyneuro-pathay) }\end{array}$ & 38 \\
\hline 31 & Ex 11 & 24222 & Missense & c. $1378 \mathrm{C}>\mathrm{T}$ & p.Arg460Cys & ? & $<10$ & $?$ & \\
\hline 32 & Ex 11 & 24285 & Missense & c. $1378 \mathrm{C}>\mathrm{T}$ & p.R460C & $\dot{\mathrm{F}}$ & $<35$ & Pure & 27 \\
\hline 33 & Ex 11 & 24228 & Missense & c. $1379 \mathrm{G}>\mathrm{A}$ & p.Arg460Cys & $\mathrm{F}$ & $<35$ & Pure & Novel \\
\hline 34 & Ex 12 & 25910 & Nonsense & c. $1417 C>T$ & p.Arg $460 \mathrm{His}$ & $F$ & $<10$ & $\begin{array}{l}\text { Autonomic nervous } \\
\text { system }\end{array}$ & Novel \\
\hline 35 & Ex 12 & 24255 & Insertion & c.1462_1463insTA & p.GIn473Term & $F$ & $>35$ & Pure & Novel \\
\hline 36 & Ex 13 & 25923 & Missense & c. $1495 \mathrm{C}>\mathrm{T}$ & $\begin{array}{l}\text { p.Arg488llef- } \\
\text { sTerm530 }\end{array}$ & $\mathrm{F}$ & $<10$ & Pure & 7 \\
\hline 37 & Ex 13 & 21929 & Missense & c. $1496 \mathrm{G}>\mathrm{A}$ & p.Arg499Cys & $\mathrm{F}$ & $<10$ & Trunk-ataxia & 28 \\
\hline 38 & Ex 13 & 19598 & Missense & c. $1507 C>T$ & p.Arg $499 \mathrm{His}$ & ? & ? & ? & 14 \\
\hline 49 & Ex 14 & 21900 & Missense & c. $1540 A>C$ & p.Arg503Trp & $\mathrm{F}$ & $?$ & $?$ & Novel \\
\hline 40 & Ex 14 & 21985 & Missense & c. $1540 A>G$ & p.Arg514Gly & $\mathrm{F}$ & $>35$ & $?$ & Novel (2) \\
\hline 41 & Ex 15 & 24230 & Insertion & c.1649_1650insCCTAAC & p.Arg514Gly & $\mathrm{F}$ & $<35$ & Pure & Novel \\
\hline 42 & Ex 15 & 19591 & Missense & c. $1664 \overline{\mathrm{A}}>\mathrm{G}$ & $\begin{array}{l}\text { p.550_551in- } \\
\text { sLeuThr }\end{array}$ & $F$ & $<35$ & Pure & Novel \\
\hline 43 & Ex 15 & 25941 & Missense & c. $1670 \mathrm{C}>\mathrm{T}$ & p.Asp555Gly & $\mathrm{F}$ & $<35$ & Pure & Novel \\
\hline 44 & Ex 15 & 21920 & Nonsense & c. $1684 C>T$ & p.Ala557 Val & $?$ & $?$ & $?$ & \\
\hline 45 & Ex 15 & 21967 & Nonsense & c. $1684 \mathrm{C}>\mathrm{T}$ & p.Arg562Term & $?$ & $>35$ & Pure & 8 \\
\hline 46 & Ex 15 & 21974 & Nonsense & c. $1684 C>T$ & p.Arg562Term & $\mathrm{F}$ & $>35$ & Pure & 8 \\
\hline 47 & Ex 15 & 24201 & Nonsense & c. $1684 C>T$ & p.Arg562Term & $\mathrm{F}$ & $<35$ & Pure & 8 \\
\hline 48 & Ex 15 & 25912 & Nonsense & c. $1684 C>T$ & p.Arg562Term & $\mathrm{F}$ & $>35$ & Pure & 8 \\
\hline 49 & Ex 16 & 19594 & Nonsense & c. $1702 C>T$ & p.Arg562Term & $?$ & $?$ & $?$ & Novel \\
\hline 50 & Ex 17 & 19597 & Missense & c. $1821 G>C$ & p.GIn568Term & $?$ & $?$ & $?$ & 13 \\
\hline 51 & Ex 17 & 25936 & Missense & c. $1821 G>C$ & p.Trp607Cys & $\mathrm{F}$ & $<35$ & Pure & 13 \\
\hline 52 & Ex 1- Ex 3 & 24278 & Exon deletion & c.1-?_682+?del & Unknown & $?$ & ? & ? & 19 \\
\hline 53 & Ex 2- Ex 9 & 21976 & Exon deletion & c.416-? 1493+?del & Unknown & $\mathrm{F}$ & $<35$ & Pure & Novel \\
\hline 54 & Ex 8 & 21968 & Exon deletion & c.1099-?_1173+?del & Unknown & $\mathrm{F}$ & $<10$ & Pure & Novel \\
\hline 55 & Ex $9-$ Ex 17 & 21940 & Exon deletion & c.1174-? $1851+? \mathrm{del}$ & Unknown & $\mathrm{F}$ & $>35$ & Pure & Novel \\
\hline 56 & Ex $2-$ Ex16 & 24270 & Exon deletion & c.416-?_1728+?del & Unknown & $\mathrm{F}$ & $>35$ & Pure & 29 \\
\hline 57 & Ex 17 & 24281 & Exon deletion & c.1729-?_18511?del & Unknown & $?$ & $?$ & $?$ & 19 \\
\hline
\end{tabular}

Note: F, Familial; S, Sporadic. 
a

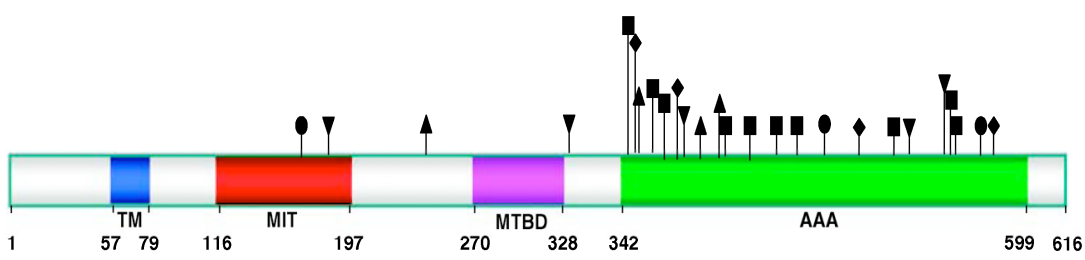

b

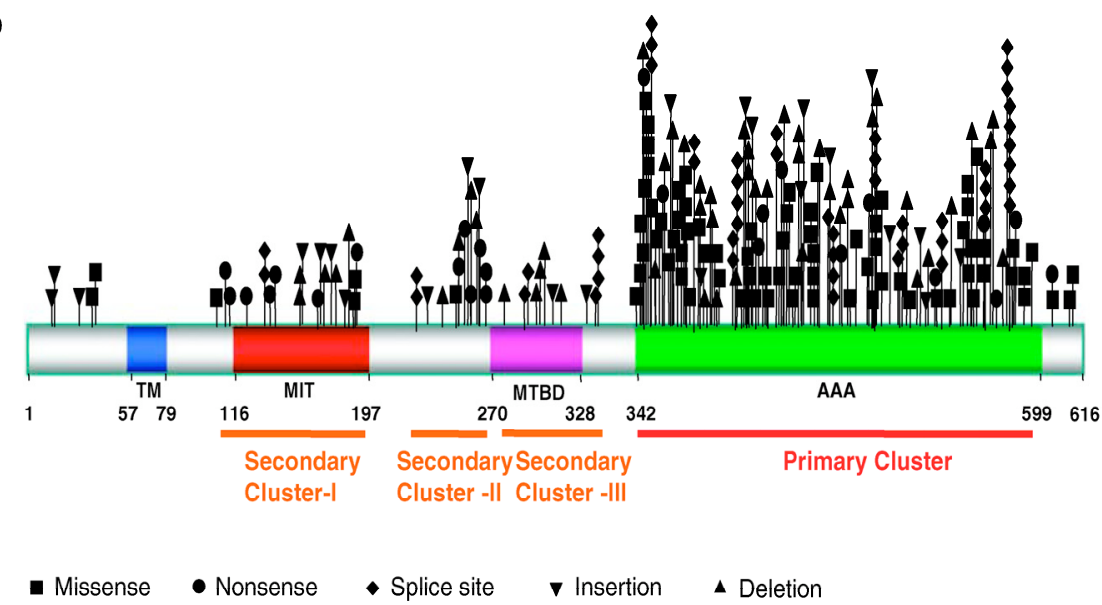

TM - Transmembrane Domain; MIT - Microtubule Interacting and Trafficking Domain

MTBD - Microtubule Binding Domain; AAA - ATPase Associated with Various Cellular Activities Domain

Figure 1 Schematic diagram representing the structural domains of the spastin protein (616 amino acids) and showing the localization of the identified mutations. The different domains are highlighted with different colors and different kinds of mutations are marked with different symbols as summarized on the left at the bottom of the figure. (a) Representation of 26 novel mutations identified in our study. Twenty-two out of 26 mutations are located in the AAA domain and two others in the MIT domain. (b) Distribution of all mutations identified to date in the spastin protein. The primary hot spot region is indicated as a red bar in the AAA domain. Three secondary hot spot regions are shown as orange bars below the spastin structure. Note: Three novel gross deletions are not included for representation; also gross deletions are excluded for distribution analysis.

the L44 allele is a genetic modifier of the HSP disease. ${ }^{10,31}$ In our cohort of $200 \mathrm{HSP}$ patients, the S44L variant was identified in six unrelated HSP cases in heterozygous state, therefore the incidence of the L44 allele in our cohort is $1.5 \%$. In addition, in two HSP individuals, we detected a second mutation in SPAST apart from the L44 allele (Table S4).

Putative genotype-phenotype correlations

To determine whether the spastin missense mutations have a different pathomechanism in contrast to other mutation types is to examine a correlation between mutation types and clinical features, such as age at onset of the symptoms. We therefore grouped the 57 identified mutations in our HSP cohort into two groups: the missense mutations (23) and the other types of mutations (34). We placed the number of identified mutations into two categories based upon age at onset of the symptoms, namely, onset before/ at 35 years $(\leq 35)$ and after 35 years $(>35)$ as represented in
Table 2 The proportions of missense and other types of mutations in different age groups, pure/complex and familial/sporadic cases of HSP

\begin{tabular}{lccc}
\hline & Missense mutations & Other mutations & All mutations \\
\hline & 23 & 34 & 57 \\
$<35$ years & 13 & 13 & 26 \\
$>35$ years & 3 & 10 & 13 \\
Unknown & 7 & 11 & 18 \\
Pure & 11 & 21 & 32 \\
Complex & 3 & 2 & 5 \\
Unknown & 9 & 11 & 20 \\
Familial & 16 & 20 & 36 \\
Sporadic & 1 & 2 & 3 \\
Unknown & 6 & 12 & 18 \\
\hline
\end{tabular}

Table 2. In the $\leq 35$ age group, we observed an equal proportion of missense mutations $(13 / 26 ; 50 \%)$ and other types of mutations $(13 / 26 ; 50 \%)$ (Table 2$)$. In the $>35$ age 
group, we detected a higher fraction of other mutation types, $(10 / 13 ; 76.9 \%)$ as compared with missense mutations $(3 / 13 ; 23 \%)$ (Table 2 ). However, statistically the differences between the proportion of missense and other mutations in $>35$ age group was not significant $(P>0.05)$. Interestingly, we noticed a skewed distribution of missense mutations between the two age groups; $\leq 35$ and $>35$ years. Therefore, we tested a null hypothesis that there is no difference in the proportion of the subjects with missense mutations between two age groups $\leq 35$ and $>35$ years, the chi-square test demonstrated that there is a significant difference $(P<0.012)$, whereas no significant difference could be detected for subjects with other mutation types $(P>0.5)$.

\section{Discussion}

In our current endeavor, we attempted to expand the mutational spectrum of SPAST. To this end, we screened for mutations in the SPAST gene in 200 HSP patients and identified 47 different mutations, out of which 29 were novel mutations. The overall frequency of SPAST mutations in our cohort was $28.5 \%(57 / 200)$. The mutation rate did not change significantly when we only considered pure HSP, which was $29.4 \%$ (32/109). Interestingly, in case of complex HSP a high mutational rate of $21.7 \%(5 / 23)$ was detected in our HSP cohort, which highlights the need to screen for the SPAST mutation in complex HSP cases. However, owing to lack of family history, we were unable to show segregation of additional symptom(s) with paraplegia, therefore, it is also possible that the complex phenotype could also be because of an independent locus other than the SPAST. The mutation detection rate in our cohort is consistent with the range of $15-44 \%$, which was observed previously in other populations. ${ }^{8-11,13}$ The frequency of mutations increased if we only considered the autosomal dominant HSP cases $(36.4 \%)$ suggesting that prevalence of SPAST mutation is higher in the familial cases. Among the sporadic cases of HSP, the frequency of mutations was $6.5 \%(3 / 46)$, which was lower than the previously reported rate of $12-18 \% .{ }^{13,14}$ This discrepancy could be because of the different population type and size. Nevertheless, from a diagnostic point of view, our and other reports emphasize the need to screen for SPAST mutations in the sporadic HSP cases.

In our HSP cohort, we identified 51 mutations in the SPAST gene by direct sequencing of all the $200 \mathrm{HSP}$ patients, which left 149 HSP cases in which no mutations could be detected by conventional sequencing. To determine gross deletion/insertion in the SPAST gene, we performed MLPA analysis in these 149 HSP cases and detected six additional mutations, which accounts for $4 \%$ (6/149) of remaining HSP cases. Previously, two independent studies used same SPAST-specific MLPA assay and reported a much higher proportion (18-20\%) of deletion in HSP patients. ${ }^{19,20}$ The observed disparity in the proportion of large deletions between our HSP cohort and others could be because of the divergence and ethnic variability in these cohorts. Nevertheless, our report of much lower proportion of gross deletions in the SPAST gene in the HSP patients stresses the need to perform MLPA in various HSP cohorts to determine the incidence rate of gross deletions in worldwide HSP populations.

It is remarkable that $22(85 \%)$ out of the 26 novel mutations (excluding the gross deletions) were located in the AAA domain of spastin. Previously, our group reported clustering of mutations in the AAA domain of spastin in a German HSP cohort ${ }^{12}$ and this clustering in AAA domain was also observed in several other HSP cohorts. ${ }^{6-10}$ Moreover, the distribution of mutations reported in the database over the structural domains of spastin outside the AAA domain were also not uniform; rather they were concentrated in certain regions of the protein, which constituted various functional domains, such as MIT and MTBD. In prior studies, exon 1 , exon 5 and exon 8 of SPAST was recognized as hot spot regions; $;^{32-34}$ however, no correlation to functional domain of spastin was implicated. Overall, it appears that different functional domains of spastin are target regions for mutations, which underlines their functional significance. Identification of these cluster regions highlights the need to set these regions as priority in the molecular diagnostic screens.

Beside a few exceptions, almost all the missense mutations in spastin are located in the AAA domain and recent studies suggest that these missense mutations might exert a dominant-negative effect on the molecular function of spastin. ${ }^{35,36}$ Utilization of a recently modeled structure of the AAA domain of spastin, ${ }^{22}$ as a framework, enabled us to classify the identified missense mutations from our cohort into different functional groups such as active site, protomer-protomer interaction, pore loop and unknown structural group of mutations. The functional categorization of the novel missense mutations, based upon the structural model of spastin will enable us in future to predict any identified sequence variant in a HSPSPAST patient as disease-causing mutation with greater level of certainty. These structural predictions of various functional classes of missense mutations need to be validated by biochemical/cellular studies and data from the structural model should be interpreted with cautiousness. However, in a recent study, we could validate at the cellular level the functional effect of two sequence variants (E442Q and R499C) of spastin, which were predicted as active site mutations from the structural model of spastin. ${ }^{22}$

The rare $\mathrm{S} 44 \mathrm{~L}$ polymorphism is considered to act as a modifier of the HSP phenotype. ${ }^{10,31,37}$ S44L is not considered as a susceptibility factor for HSP because its

European Journal of Human Genetics 
frequency rate is similar in HSP patients and controls. ${ }^{14}$ In our study, we could not ascertain the role of S44L (heterozygous state alone) on manifestation of HSP. It is possible that the patients heterozygous for S44L might have another mutation in spastin, which could not be identified by our screen or might have a mutation in a different HSP gene.

No apparent genotype-phenotype correlation is evident among missense mutations and other SPAST mutations. ${ }^{8,14,38}$ Although several studies indicated that missense mutation might act in a dominant-negative fashion in contrast to other mutations, which lead to a loss of function. To determine, whether missense mutation leads to early onset of HSP, we assorted our HSP cohort into two different groups based upon AAO ( $\leq 35$ and $>35$ years). The rational behind sorting our HSP cohort into these two age groups was derived from Harding's classification of HSP patients into two distinct groups, early age onset $(\leq 35$ years) and late age onset ( $>35$ years). ${ }^{39}$ This AAO $(\leq 35$ and $>35$ years) classification was also used by Fonknechten and coworkers for determination of genotype-phenotype correlation. ${ }^{8}$ We observed an obvious difference in the proportion of mutations between the missense group as compared with the other types of mutations in age group of $>35$ years. However, the observed difference was not statistically significant $(P>0.05)$ because of a very small sample size. Remarkably, we could reject a null hypothesis that there is no difference in the proportion of subjects for missense mutations between two age groups, $\leq 35$ and $>35$ years, which was statistically significant $(P<0.0124)$. Our data show a tentative genotype-phenotype correlation and suggest that in case of missense mutations the onset of phenotype is earlier. Owing to a small sample size, this correlation between AAO and missense mutation should be interpreted with discretion. Previously, early $\mathrm{AAO}$ in patients with missense mutation was also reported; however, this study only accounted for two missense mutations out of a total five mutations. ${ }^{40}$ Moreover, in a meta-analysis $^{38}$ no significant correlation between AAO and mutational class was evident, but one limitation of this study was the sample size. Nevertheless, these different pathomechanism modes, such as loss of function and dominant-negative function for different classes/types of spastin mutations need to be carefully resolved by experimental means; otherwise there will be repercussions on the likely success of any therapeutical approach devised for spastin-associated HSP.

\section{Acknowledgements}

We thank the HSP patients/families for their participation in this study, B Brandt for excellent technical assistance, P Grzmil for statistical analysis and $W$ Engel for critical comments/reading of the paper. This work was funded partly by a Tom Wahlig Stiftung Grant and the Deutsche Forschungsgemeinschaft (MA 3344/2-1) Grant to $A U M$ and by the institution's internal fund.

\section{References}

1 Bruyn RPM, Scheltens P: Hereditary spastic paraparesis (Strümpell-Lorrain), Diseases of the motor system; in de Jong JMBV (ed): Handbook of Clinical Neurology. Elsevier Science Publishers: Oxford, 1991, vol 15, pp 301-318.

2 Behan WM, Maia M: Strumpell's familial spastic paraplegiã: genetics and neuropathology. J Neurol Neurosurg Psychiatry 1974; 37: 8-20.

3 Schwarz GA, Liu CN: Hereditary (familial) spastic paraplegia; further clinical and pathologic observations. AMA Arch Neurol Psychiatry 1956; 75: 144-162.

4 Fink JK: The hereditary spastic paraplegias: nine genes and counting. Arch Neurol 2003; 60: 1045-1049.

5 Reid E: Science in motion: common molecular pathological themes emerge in the hereditary spastic paraplegias. J Med Genet 2003; 40: $81-86$.

6 Hazan J, Fonknechten N, Mavel D et al: Spastin, a new AAA protein, is altered in the most frequent form of autosomal dominant spastic paraplegia. Nat Genet 1999; 23 : 296-303.

7 McDermott CJ, Burness CE, Kirby J et al: Clinical features of hereditary spastic paraplegia due to spastin mutation. Neurology 2006; 67: 45-51.

8 Fonknechten N, Mavel D, Byrne P et al: Spectrum of SPG4 mutations in autosomal dominant spastic paraplegia. Hum Mol Genet 2000; 9: 637-644.

9 Hentati A, Deng HX, Zhai $\mathrm{H}$ et al: Novel mutations in spastin gene and absence of correlation with age at onset of symptoms. Neurology 2000; 55: 1388-1390.

10 Lindsey JC, Lusher ME, McDermott $\mathrm{CJ}$ et al: Mutation analysis of the spastin gene (SPG4) in patients with hereditary spastic paraparesis. JMed Genet 2000; 37: 759-765.

11 Patrono $\mathrm{C}$, Scarano V, Cricchi $\mathrm{F}$ et al: Autosomal dominant hereditary spastic paraplegia: DHPLC-based mutation analysis of SPG4 reveals eleven novel mutations. Hum Mutat 2005; 25 : 506.

12 Sauter S, Miterski B, Klimpe $S$ et al: Mutation analysis of the spastin gene (SPG4) in patients in Germany with autosomal dominant hereditary spastic paraplegia. Hum Mutat 2002; 20 : 127-132.

13 Crippa F, Panzeri C, Martinuzzi A et al: Eight novel mutations in SPG4 in a large sample of patients with hereditary spastic paraplegia. Arch Neurol 2006; 63: 750-755.

14 Depienne C, Tallaksen C, Lephay JY et al: Spastin mutations are frequent in sporadic spastic paraparesis and their spectrum is different from that observed in familial cases. J Med Genet 2006; 43: $259-265$

15 Confalonieri F, Duguet M: A 200-amino acid ATPase module in search of a basic function. Bioessays 1995; 17: 639-650.

16 Frickey T, Lupas AN: Phylogenetic analysis of AAA proteins. J Struct Biol 2004; 146: 2-10.

17 Patel S, Latterich M: The AAA team: related ATPases with diverse functions. Trends Cell Biol 1998; 8: 65-71.

18 Hanson PI, Whiteheart SW: AAAt proteins: have engine, will work. Nat Rev Mol Cell Biol 2005; 6: 519-529.

19 Beetz C, Nygren AO, Schickel J et al: High frequency of partial SPAST deletions in autosomal dominant hereditary spastic paraplegia. Neurology 2006; 67: 1926-1930.

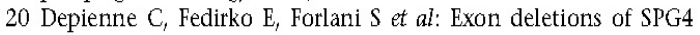
are a frequent cause of hereditary spastic paraplegia. J Med Genet 2007; 44: 281-284.

21 Harding AE: Classification of the hereditary ataxias and paraplegias. Lancet 1983; 1: 1151-1155.

22 Pantakani DV, Swapna LS, Srinivasan N, Mannan AU: Spastin oligomerizes into a hexamer and the mutant spastin (E442O) redistribute the wild-type spastin into filamentous microtubule. J Neurochem 2008; 106: 613-624.

23 Meijer IA, Hand CK, Cossette P et al: Spectrum of SPG4 mutations in a large collection of North American families with hereditary spastic paraplegia. Arch Neurol 2002; 59: 281-286. 
24 Schickel J, Beetz C, Frommel C et al: Unexpected pathogenic mechanism of a novel mutation in the coding sequence of SPG4 (spastin). Neurology 2006; 66: 421-423.

25 Svenson IK, Ashley-Koch AE, Gaskell PC el al: Identification and expression analysis of spastin gene mutations in hereditary spastic paraplegia. Am J Hum Genet 2001; 68: 1077-1085.

26 Mead SH, Proukakis C, Wood N et al: A large family with hereditary spastic paraparesis due to a frame shift mutation of the spastin (SPG4) gene: association with multiple sclerosis in two affected siblings and epilepsy in other affected family members. J Neurol Neurosurg Psychiatry 2001; 71: 788-791.

27 Falco M, Scuderi C, Musumeci S et al: Two novel mutations in the spastin gene (SPG4) found by DHPLC mutation analysis. Neuromuscul Disord 2004; 14: 750-753.

28 Park SY, Ki CS, Kim HJ et al: Mutation analysis of SPG4 and SPG3A genes and its implication in molecular diagnosis of Korean patients with hereditary spastic paraplegia. Arch Neurol 2005; 62: $1118-1121$.

29 Beetz C, Zuchner S, Ashley-Koch A et al: Linkage to a known gene but no mutation identified: comprehensive reanalysis of SPG4 HSP pedigrees reveals large deletions as the sole cause. Hum Mutat 2007; 28: 739-740.

30 White SR, Evans KJ, Lary J, Cole JL, Lauring B: Recognition of C-terminal amino acids in tubulin by pore loops in Spastin is important for microtubule severing. I Cell Biol 2007; 176 $995-1005$.

31 Svenson IK, Kloos MT, Gaskell PC et al: Intragenic modifiers of hereditary spastic paraplegia due to spastin gene mutations. Neurogenetics 2004; 5: 157-164.

32 McDermott CJ, Burness CE, Rao Kirby J, Shaw PJ: Further insights into HSP and spastin: severe complicated phenotypes and evidence of lower motor neurone dysfunction (Abstract number 059);Proceedings of Clinical Neurosciences, Torquay, Devon, 7-9 September 2005; neurology abstracts only. I Neurol Neurosurg Psychialry 2006; 77: 126-142.

33 Nielsen JE, Koefoed P, Kjaergaard S, Jensen LN, Nørremølle A, Hasholt L: Prenatal diagnosis of autosomal dominant hereditary spastic paraplegia (SPG4) using direct mutation detection. Prenat Diagn 2004; 24: 363-366.

34 Zhao GH, Tang BS, Luo Wet al: Spastin gene mutation in Chinese patients with hereditary spastic paraplegia. Zhonghua Yi Xue Yi Chuan Xue Za Zhi 2003; 20: 177-180. (article in Chinese).

35 Errico A, Ballabio A, Rugarli EI: Spastin, the protein mutated in autosomal dominant hereditary spastic paraplegia, is involved in microtubule dynamics. Hum Mol Genet 2002; 11: 153-163.

36 Evans KJ, Gomes ER, Reisenweber SM, Gundersen GG, Lauring BP: Linking axonal degeneration to microtubule remodeling by Spastin-mediated microtubule severing. I Cell Biol 2005; 168 599-606.

37 Chinnery PF, Keers SM, Holden MJ, Ramesh V, Dalton A: Infantile hereditary spastic paraparesis due to codominant mutations in the spastin gene. Neurology 2004; 63: 710-712.

38 Yip AG, Durr A, Marchuk DA et al: Meta-analysis of age at onset in spastin-associated hereditary spastic paraplegia provides no evidence for a correlation with mutational class. I Med Genet 2003; 40: e106.

39 Harding AE: Hereditary 'pure' spastic paraplegia: a clinical and genetic study of 22 families. J Neurol Neurosurg Psychiatry 1981; 44: 871-883.

40 Ivanova N, Löfgren A, Tournev I et al: Spastin gene mutations in Bulgarian patients with hereditary spastic paraplegia. Clin Genet 2006; 70: 490-495.

Supplementary Information accompanies the paper on European Journal of Human Genetics website (http://www.nature.com/ejhg) 


\subsection{ZFYVE27 (Protrudin) oligomerize as dimer/tetramer and truncated ZFYVE27 $\left(\triangle \mathrm{HR3}^{184-208}\right)$ deters the ability of wild type ZFYVE27 to promote neurite extension}

This part of the thesis describes about ZFYVE27 (Protrudin), a spastin interacting protein, which homo-oligomerizes into dimer/tetramer. In a yeast two-hybrid screen, we observed that ZFYVE27 can interact with itself. Further studies indicated that the core interaction region lie within the third hydrophobic region (HR3) of the protein. Furthermore, co-localization and co-immunoprecipitation experiments confirmed ZFYVE27 self-interaction and also indicated that additional unknown functional regions provide stability to the oligomeric ZFYVE27 structure. Using various biochemical methods, we showed that ZFYVE27 is a peripheral membrane protein; binds to phosphorylated derivatives of phosphatidylinositol (PtdIns) lipid molecules and exists as dimer/tetramer. Although, the core interaction region, HR3 is dispensable for selfassociation of ZFYVE27, it is essential for neurite extension. Further, co-expression of truncated ZFYVE27 ( $\left.\triangle \mathrm{HR} 3^{184-208}\right)$ hampers the function of wild type ZFYVE27, suggesting the dominant negative effect of truncated ZFYVE27.

D. V. Krishna Pantakani and Ashraf U. Mannan

Status: Manuscript is in submission stage

\section{Author contributions to the work:}

1. D. V. Krishna Pantakani: Designed and performed the experiments. Involved in the manuscript preparation.

2. Ashraf U. Mannan: Conceived and designed the experiments. Interpretated the data and prepared the manuscript. 


\title{
ZFYVE27 (Protrudin) oligomerize as dimer/tetramer and truncated ZFYVE27
}

\section{$\left(\triangle H R 3^{184-208}\right)$ deters the ability of wild type ZFYVE27 to promote neurite extension}

\author{
Krishna Pantakani D. V. ${ }^{1}$ and Ashraf U. Mannan ${ }^{1,2, \S}$
}

${ }^{1}$ Institute of Human Genetics, University of Goettingen, Goettingen, Germany.

${ }^{2} D F G$-Research Center for Molecular Physiology of the Brain (CMPB), Goettingen, Germany.

ZFYVE27/Protrudin was initially identified as an interacting protein of spastin, the most commonly mutated protein in hereditary spastic paraplegia. We now identified that ZFYVE27 interacts with itself by yeast-two hybrid screen and show that ZFYVE27 core interaction region resides within the third hydrophobic region (HR3). The observed oligomerization of ZFYVE27 was further confirmed in mammalian cells by co-immunoprecipitation and co-localization thus validating the interaction in physiological conditions. Sucrose gradient centrifugation and immunoblot analysis demonstrate that ZFYVE27 assembles into homo-dimer/tetramer and also forms SDS-resistant dimers. Membrane association studies revealed that ZFYVE27 is a peripheral membrane protein and can bind specifically to all the phosphorylated derivatives of phosphatidylinositol (PtdIns) lipid molecules. Further, we show that spastin associates with membrane as a peripheral membrane protein. In silico analysis on FYVE domain of ZFYVE27 designate that it might belongs to a novel sub-family of FYVE proteins, as it can bind specifically to all the phosphorylated derivatives of PtdIns and not been having three signature motifs of a typical FYVE domain: the N-termianl WXXD, the central $R(R / K) H H C R$ and the C-terminal RVC motifs. Finally, we show that although the core interaction region, HR3 is dispensable for self-association of ZFYVE27, it is essential for neurite formation and co-expression of the truncated ZFYVE27 hampers the function of wild type ZFYVE27.

Spastin encoded by SPAST gene, is a microtubule (MT) severing enzyme and belongs to AAA (ATPase Associated with various cellular Activities) family of proteins and is mutated in about $40 \%$ of autosomal dominant hereditary spastic paraplegia (AD-IISP) cases (1-8). Significant insights into the molecular function of spastin were derived from the identification

${ }^{\S}$ To whom correspondence should be addressed: Dr. Ashraf U. Mannan, Institute of Human Genetics, University of Goettingen, Heinrich-Dueker-Weg 12, D-37073, Goettingen, Germany, Phone: +49-551-397522, Fax: +49-551399303, E-mail: amannan@gwdg.de

The abbreviations used are: HSP, hereditary spastic paraplegia; HR, hydrophobic region; PtdIns, phosphatidylinositol; Y2H, yeast-two hybrid; SDS, sodium dodecyl sulfate; TM, transmembrane. and characterization of spastin interacting proteins namely reticulon1, atlastin, CHMP1B, ZFYVE27 and NA14 (9-13). Apart from NA14, all the spastin interacting proteins identified till to date are components of vesicular/membrane trafficking. Recently, overexpression of spastin has been shown to promote the neurite outgrowth (axonal branch formation) in neurons, conversely depletion of spastin from neurons resulted in the significant reduction of axonal branch formation (14), thus correlating the MT severing function of spastin in neuritogenesis/neuronal homeostasis.

ZFYVE27/Protrudin, a member of FYVE (Fab1p, YOTB, $\underline{\text { Vaclp }}$ and EEA1) finger family of proteins, was initially identified as a spastin interacting protein (12). The role of ZFYVE27 in a molecular process relevant to HSP is highlighted by recent functional studies in PC12 cells and showed that ZFYVE27 plays a vital function in neuronal differentiation and along with Rab11 plays a central role in directional membrane trafficking and neurite formation (15). Interestingly, overexpression of ZFYVE27 even in non-neuronal cells such as HeLa cells induced the neurite like extensions, whereas downregulation of ZFYVE27 in PC12 cells resulted in the membrane spreading in all directions (15). More recently, ZFYVE27 was shown to interact with FKBP38 (16), which belongs to immunophilin family of proteins and members of this family regulates the folding and export of other proteins (17). Moreover, ZFYVE27 was shown to be hyperphosphorylated in FKBP38 ${ }^{-/ 2}$ mice, these mice die soon after the birth and exhibited defects in neural tube closure and abnormal nerve extensions, thus suggesting that FKBP38 regulates the ZFYVE27 phosphorylation and its ability to induce neurite formation (16). In a more recent study (18), ZFYVE27 was reported to interact with VAP (VAMP (vesicle associated membrane protein)-associated protein) via FFAT motif and this interaction was also shown to be necessary for ZFYVE27 sub-cellular localization and function in neurite outgrowth.

In the database, ZFYVE27 was classified as a FYVE domain containing protein 27 with three putative transmembrane (TM) domains or hydrophobic regions (HR), as it preserves the eight conserved cystine residues which co-ordinate the binding of two zinc ions in a cross-braced topology (19-21). Moreover, the typical FYVE domain ( 70-residue module) also contains three conserved motifs: the $\mathrm{N}$-termianl WXXD, the central R(R/K)HHCR and the C-terminal RVC motifs, which specifically recognizes 
phosphatidylinositol 3-phosphate (PtdIns3P) and targets many cytosolic proteins to PtdIns3P enriched endosomal membranes $(19,20,22-24)$. Moreover, the targeting of a number of FYVE proteins to the membrane is greatly influenced by the bivalent or multivalent PtdIns3P interactions and is greatly enhanced by the oligomerization. The FYVE domain containing EEA1 has been shown to form parallel homo-dimers via their coiled-coil regions $(23,25)$. The homo-dimerization of EEA1 was shown to juxtapose two C-terminal FYVE domains thus allowing simultaneous interactions with two PtdIns3P head groups (23). The intrinsically formed stable dimers and higher ordered oligomerization by the FYVE domain of SARA has shown to be important for the endosomal localization $(26,27)$. Moreover, a distinct antiparallel homo-dimer has been observed for FYVE domain of Drosophila Hrs, based on the structural information (28).

To decipher the molecular function of ZFYVE27, we performed a yeast-two hybrid screen on human ZFYVE27 and identified ZFYVE27 as a prey clone, suggesting that it can interact with itself. In the present study, we demonstrate the oligomerization of ZFYVE27, which is mediated mainly via its third hydrophobic region (HR3), though this region is dispensable for interaction, it is essential for neurite formation. Further, we show that ZFYVE27 is a peripheral membrane protein and can bind to all the phosphorylated derivatives of PtdIns3P. The membrane association properties of spastin were determined and show that it is also a peripheral membrane protein.

\section{EXPERIMENTAL PROCEDURES}

Yeast-two hybrid screen- We used the Matchmaker Two-Hybrid System kit (BD Clontech) for identification of putative ZFYVE27 interacting proteins. To generate the bait construct, the full-length human ZFYVE27-isoform 4 (GenBank accession No.: NM 001002262.2) was PCR amplified from human fetal brain cDNA and cloned into EcoR I and BamH I restriction sites of pGBKT7 vector (BD Clontech), resulting in a fusion of GAL4 DNA-binding domain with ZFYVE27. The pGBKT7-ZFYVE27 construct was transformed into AH109 yeast strain to exclude the no auto-activation of GAL4 activated reporter genes, HIS3, $A D E$, lac $Z$. The yeast two-hybrid screen was performed on a human fetal brain cDNA library (constructed in pGADT7-Rec plasmid) using a Matchmaker pretransformed kit (Clontech). The matchmaker pretransformed library in yeast strain Y187 was mixed and mated together with strain AH109 containing the ZFYVE27 bait construct. After 24 hours of mating, the culture was spread on SD/-Leu/-Trp/-His/-Ade plates and the surviving colonies were verified on $\mathrm{SD} /$-Leu/Trp/-His/-Ade/+ X- $\alpha$-Gal. The positive clones that were blue on $\mathrm{SD} /$-Leu/-Trp/-His/-Ade/+ X- $\alpha$-Gal were cultured and the plasmid DNA was isolated using QIAprep Spin Miniprep Kit (Qiagen). To select the transformants containing only the prey/library plasmid, the yeast plasmids from prey clones were transformed into E. coli. The cDNA inserts of these clones were PCR amplified and sequenced. Identities of individual clones were determined by BLAST analysis at the website http://blast.ncbi.nlm.nih.gov/Blast.cgi.

Direct Yeast-two hybrid assay- For the mapping of ZFYVE27 self-interaction region, additional baits and preys of ZFYVE27 with various deletions were generated in both pGBKT7 and pGADT7 vectors. The complete ORF of ZFYVE27 and truncated ZFYVE27 sequences, i.e. 1-335, at the protein level [corresponding nucleotides (nt) 201-1205 of NM 001002262.2], 110335 (nt 528-1205), 150-250 (nt 648-939), 174-218 (nt 720-854), 1-184 (nt 201-752) and 208-404 (nt 8221412) were cloned into both pGBKT7 and pGADT7 vectors. For assaying the self-interaction of ZFYVE27, bait and prey constructs were co-transformed in yeast strain AH109 by lithium acetate method (29). Cotransformants selected on $\mathrm{SD} /$-Leu/-Trp plates were tested for the reporter gene expression on $\mathrm{SD} /-\mathrm{Leu} /-$ Trp/-His/-Ade and X- $\alpha$-Gal assay.

Construction of mammalian expression plasmidsThe pGBKT7-ZFYVE27 served as a template for the PCR amplification and cloning of ZFYVE27 into $\mathrm{BamH}$ I and Kpn I restriction sites of pQM-N-Tag A (Abcam) to generate E2-hZFYVE27-WT construct. To generate c-Myc-hZFYVE27-WT construct, ZFYVE27 ORF was cloned into $E c o R$ I site of pCS2-myc expression vector (30). The E2-hZFYVE27 was used as a template for PCR amplification of a plasmid lacking HR3 (nt 750822 of NM_001002262.2) using a modified QuickChange ('Stratagene) based PCR reaction with primers complementary to the both 5 ' and 3 ' flanking regions of HR3 and generated the E2-ZFYVE27- $\triangle$ HR3 construct. For the generation of E2-ZFYVE27- $\Delta C^{1-150}$ (nt 201-650) and E2-hZFYVE27- $\Delta \mathrm{N}^{150-404}$ (nt 6431412), the PCR amplified DNA fragments were cloned into BamH I/Kpn I restriction sites of pQM-N-Tag A vector. Spastin-GFP construct was a kind gift from Dr. Elena I. Rugarli (1).

Cell culture, immunofluorescence and immunoprecipitation experiments- The NIH-3T3 and HeLa cells were cultured and maintained as previously described (11). NSC34, a mouse embryonic spinal cord-neuroblastoma hybrid cell line with a motor neuronal properties $(31,32)$ was a kind gift from Dr J. Weishaupt (University of Göttingen). The cells were cultured and maintained in Dulbecco's modified Eagle's medium (DMEM) supplemented with $10 \%$ fetal bovine serum (FBS) and 1\% penicillin/ streptomycin/ glutamine solution as previously reported (31). Primary cultures of mouse hippocampal neurons were prepared from postnatal day 2-5 mouse pups. Briefly, hippocampi were carefully dissected and tissue pieces were trypsinized and dissociated using fire-polished Pasteur pipettes. The cell suspension was seeded on poly-D-Lysine/Laminin coated coverslips (BD Biosciences) and maintained in a culture medium containing DMEM supplemented with 5\% horse serum, 
$2 \mathrm{mM}$ Glutamax, $100 \mathrm{~g} / \mathrm{ml}$ transferrin and $200 \mathrm{~g} / \mathrm{ml}$ insulin. For all the cell lines, transfection was done using Lipofectamine2000 reagent (Invitrogen) according to the manufacturer's instructions. Immunofluorescence and immunoprecipitation experiments were essentially performed as previously described $(11,33)$.

Subcellular fractionation and Triton X-114 membrane phase separation-Subcellular fractionation was performed with NSC34 cells, if necessary transiently transfected with construct. The cells were suspended in a buffer containing $20 \mathrm{mM}$ HEPES $\mathrm{pH}$ $7.8,150 \mathrm{mM} \mathrm{NaCl}$ and protease inhibitor cocktail and were triturated by passing through 27 -gauge syringe (15-20 times). The homogenate was centrifuged at $4^{\circ} \mathrm{C}$ for $5 \mathrm{~min}$ at $3,000 \mathrm{~g}$ to obtain post nuclear supernatant and the resulting supernatant was then centrifuged at $100,000 \mathrm{~g}$ for $60 \mathrm{~min}$ in a Beckman ultracentrifuge. The supernatant containing the cytosolic fraction (S-100) was separated and the pellet containing membrane fraction (P-100) was resuspended with the buffer to the original volume. Each sample $(20 \mu \mathrm{l})$ was then resolved in SDS-PAGE and immunoblotting was performed with either ZFYVE27 or GFP antibody.

The Triton X-114 phase separation was essentially performed as described previously (34). Briefly, the membrane fraction (prepared as above) was mixed with pre-condensed Triton X-114 to a final concentration of $2 \%$ and incubated on ice for $30 \mathrm{~min}$ with occasional mixing. The detergent soluble fraction obtained after centrifugation at $16,000 \mathrm{~g}$ for $15 \mathrm{~min}$ at $4^{\circ} \mathrm{C}$ was subjected to aqueous and detergent phase separation at cloudy point temperature of $37^{\circ} \mathrm{C}$ for $5 \mathrm{~min}$ and centrifuged at room temperature for $5 \mathrm{~min}$ at $16,000 \mathrm{~g}$. The detergent phase containing integral proteins and aqueous phase containing peripheral proteins were separated and subjected to one more round of separation. Finally, detergent and aqueous phases were pooled separately and the proteins were precipitated using standard acetone precipitation method. After acetone precipitation, the protein pellets were resuspended and denatured in an original volume of SDS-PAGE loading buffer and $20 \mu \mathrm{l}$ of each fraction was analyzed by SDS-PAGE and immunoblotting.

Lipid-protein overlay assay- For the protein lipid overlay assay, the ZFYVE27 containing 150-404 a.a was PCR amplified and cloned into pET28a vector (Novagen). The recombinant His-ZFYVE27 $7^{150-404}$ was purified from E. coli (BL21) using His affinity tag. A nitrocellulose membrane with immobilized phospholipids (PIP strips, Echelon Biosciences) was blocked for 1 hour in blocking buffer [3\% fatty acidfree BSA in TBST (50 mM Tris- $\mathrm{HCl}, \mathrm{pH}-7.5,150 \mathrm{mM}$ $\mathrm{NaCl}$ and $0.1 \%$ Tween 20)]. The membrane was then incubated with $200-500 \mathrm{ng} / \mathrm{ml}$ of recombinant HishZFYVE2 $7^{150-404}$ protein for overnight with gentle shaking and thereafter was washed three times $(10 \mathrm{~min}$ each) with blocking buffer. After washing, the membrane was incubated with His antibody (Qiagen) followed by three times washing and incubation with secondary antibody conjugated with HRP.

Sucrose density gradient experiments - For sucrose density gradient centrifugation, the NIH-3T3 cells overexpressing E2-ZFYVE27-WT were washed two times (10 $\mathrm{min}$ each) with PBS and incubated with HKME buffer (25 mM HEPES, pH 7.8, $150 \mathrm{mM}$ potassium acetate, $2.5 \mathrm{mM}$ magnesium acetate, $1 \mathrm{mM}$ EDTA and protease inhibitor cocktail) containing $1 \%$ Big-CHAPS for $15 \mathrm{~min}$. The cells were then harvested by scrapping the attached cells from plastic surface of culture flask and incubated for further $30 \mathrm{~min}$ on ice with mild vortexing for complete lysis. The cell debris from the protein lysate was clarified by centrifugation at $10,000 \mathrm{~g}$ for $10 \mathrm{~min}$ at $4^{\circ} \mathrm{C}$ and $200 \mu \mathrm{l}$ of the resultant supernatant was applied on 5-30\% sucrose gradients and centrifuged at $135,000 \mathrm{~g}$ for 4 hours at $25^{\circ} \mathrm{C}$ on a Beckman coulter TLS55 rotor. Approximately thirty gradient fractions of each $50 \mu \mathrm{l}$ were collected from top to bottom and $20 \mu \mathrm{l}$ of the indicated samples were analyzed by standard SDS-PAGE followed by immunoblotting with ZFYVE27 antibody. Molecular masses were calculated from the sedimentation of standard molecular weight markers: albumin, bovine serum $(\sim 66 \mathrm{kDa})$, Alcohol dehydrogenase, yeast $(\sim 150$ $\mathrm{kDa})$ and apoferritin, horse spleen $\left(\begin{array}{rlll}\sim 50 & \mathrm{kDa}\end{array}\right)$ purchased from (Sigma-Aldrich).

\section{RESULTS}

ZFYVE27 interacts with itself primarily through its third hydrophobic region- We performed a yeast-two hybrid (Y2H) screen on human fetal brain cDNA using full-length human ZFYVE27 as a bait protein in an attempt to identify interacting proteins of ZFYVE27. One of the interacting protein that we have obtained was ZFYVE27 (full-length) itself, suggesting that it could form homo-oligomers (Fig. 1B). To confirm the homooligomerization of ZFYVE27 and to identify the core interaction region, we generated several deletion constructs of ZFYVE27 (Fig. 1A) and tested their ability for interaction in direct-Y2H assay with either full-length (Fig. 1C and supplementary Fig. S1A) or with deletion constructs (supplementary Fig. S1B). Direct-Y2H assay validated the interaction of full-length ZFYVE27 with itself (Fig. 1C). We were also able to identify the core interaction region to be the third hydrophobic region (HR3) as ZFYVE27 $7^{150-250}$ consisting of HR3 alone could interact with full length (Fig. 1C) and the deletion of this region $(\triangle H R 3)$ in ZFYVE27 ${ }^{1-335}$ failed to interact with full-length ZFYVE27 (Fig. 1D). The deletion construct ZFYVE2 $7^{208-404}$ containing FYVE domain and coiledcoil region, which usually favors the oligomerization, failed to interact with full length construct as well with itself, thus excluding this region as the core interaction region (Fig. 1C and supplementary Fig. S1A and B). The deletion construct ZFYVE27 $7^{1-184}$ also showed weak interaction with full-length ZFYVE27 (Fig. 1C), but failed to interact with itself (supplementary Fig. S1B). To investigate the role of ZFYVE2 $7^{1-184}$ in self- 
A.

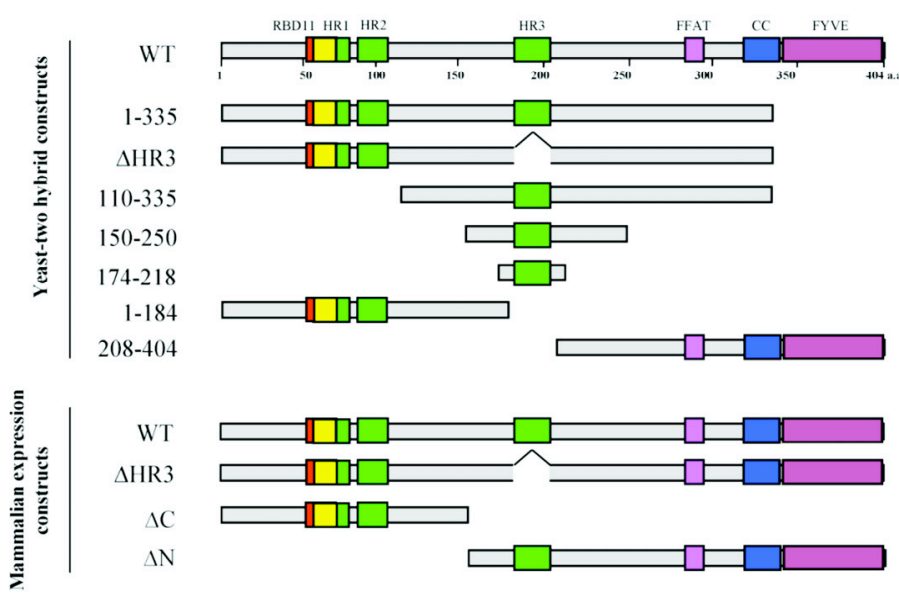

B.
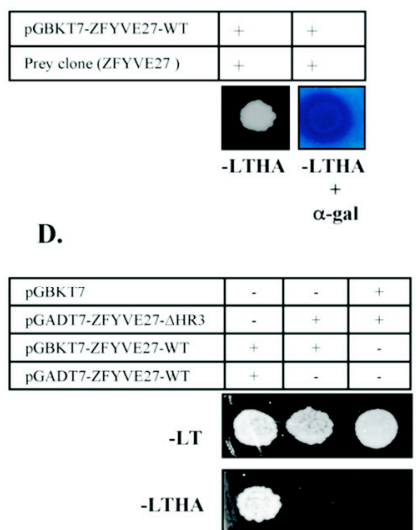

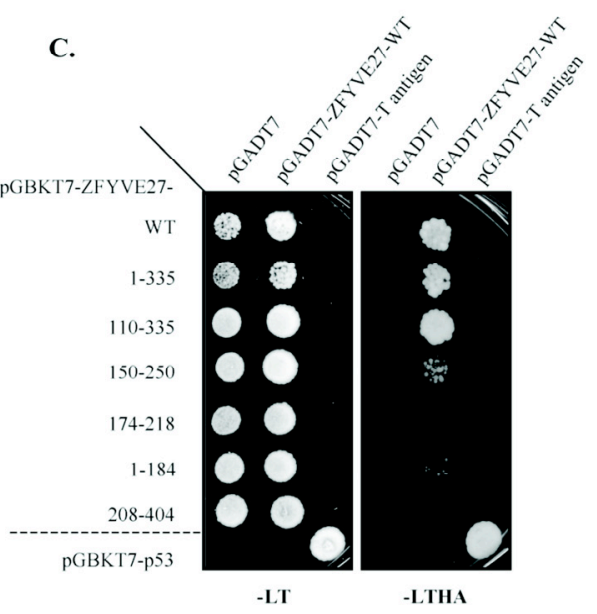

Figure 1. ZFYVE27 interacts with itself. (A) Schematic diagram showing protein domain organization and delineation of ZFYVE27 constructs. ZFYVE27 contains Rab11 binding domain RBD11 (red) at its N-terminus and FYVE domain (purple) at C-terminus. Three hydrophobic regions (HR) are highlighted in green and that of the HR1 region overlaps (yellow) with the RBD11 motif. The FFAT motif (pink), which mediates interaction with VAP-A and the coiled-coil region (blue) are indicated. The generated deletion constructs for yeasttwo hybrid analysis and mammalian expression constructs are depicted thereof. (B) Activation of GAL4 reporter genes by interaction of ZFYVE27 with the prey clone (ZFYVE27) in yeast-two hybrid (Y2H) screen. A robust growth of yeast strain AH109 was observed on the nutritional selection medium -LTHA (lacking leucine, tryptophan, histidine and adenine) and the $\alpha$-galactosidase ( $\alpha$-gal) activity. (C) Determination of the core interaction region of ZFYVE27 self-interaction by direct-Y2H. The indicated deletion constructs of ZFYVE27 were fused with DNA binding domain of GAL4 and evaluated for their ability to interact with full-length ZFYVE27 fused to activation domain of GAL4 in Y2H experiments. The reporter yeast, AH109 co-transformed with various deletion constructs and with either vector alone (pGADI7) or with full-length ZFY VE27 in pGADI7 were spotted on nutritional medium lacking tryptophan and leucine (-LI) or lacking tryptophan, leucine, histidine and adenine (-LTHA). The HIS3 and ADE reporter genes activation occurs by transcriptional activation of GAL4, which is indicative of interaction between full-length ZFYVE27 and various deleted forms of ZFYVE27. The interaction between $\mathrm{p} 53$ and T-antigen was used as a positive control inY2H assay. (D) Evaluation of truncated ZFYVE27 (deletion of HR3 in ZFYVE27 $7^{1-335}$ construct) interaction with full-length ZFYVE27.

association of ZFYVE27, we made two deletion constructs devoid of hydrophobic regions (HR1 \& HR2) and analyzed for their ability to interact with full-length ZFYVE27 or with itself. Interestingly, these two deletion constructs failed to interact with full-length ZFYVE27, but showed the interaction with itself and also indicated the cross talk between these two regions, as ZFYVE27 $7^{1-71}$ could interact with ZFYVE2 $7^{110-184}$ (Supplementary Fig. 1B).

Core interaction region of ZFYVE27 is dispensable for its homo-oligomerization- To validate the observed
ZFYVE27 self-interaction in direct-Y2H assay, we performed co-immunoprecipitation experiments with full-length ZFYVE27 tagged with either E2 or c-Myc tag (E2-ZFYVE27-WT or c-Myc-ZFYVE27-WT) together with various deletion constructs tagged with E2 (Fig. 1A) using mammalian cell culture approach. Coexpression of E2-ZFYVE27-WT and c-Myc-ZFYVE27WT in NIH-3T3 cells and subsequent coimmunoprecipitation revealed that ZFYVE27 indeed could interact with itself (Fig. 2). Next, we checked the ability of various deletion constructs to interact with 
wild type ZFYVE27 in overexpression experiments in NIH-3T3 cells. Surprisingly, the E2-ZFYVE27- $\Delta$ C also showed a weak interaction with full-length as compared to the E2-ZFYVE27- $\Delta \mathrm{N}$, which is expected to interact, as it contains the core interaction region, HR3 (Fig. 2). Further, to validate the role of HR3 as the core interaction region of ZFYVE27, we generated a deletion construct, E2-ZFYVE27- $\triangle \mathrm{HR} 3$ and assayed its ability to interact with c-Myc-ZFYVE27-WT. Surprisingly, E2-ZFYVE27- $\triangle$ HR3 showed weak interaction with cMyc-ZFYVE27-WT (Fig. 2).

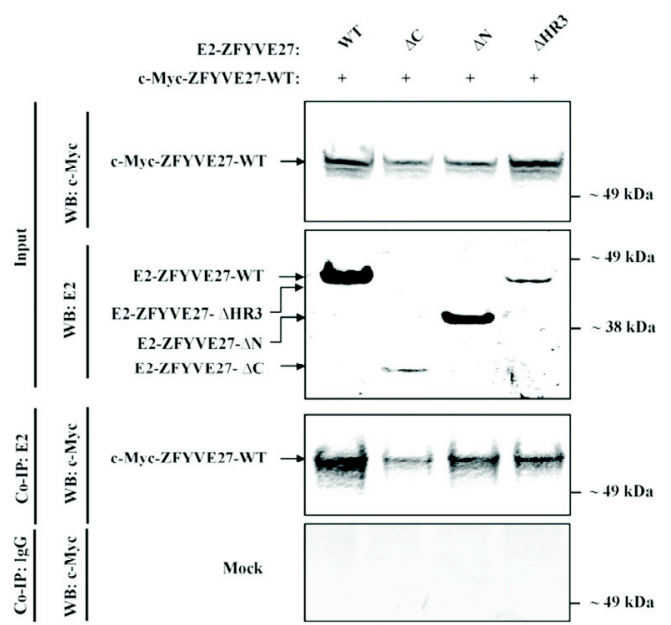

Figure 2. Co-immunoprecipitation assay to validate interaction of ZFYVE27 in mammalian cells. (A) The full-length human ZFYVE27 fused with c-Myc epitope tag was used to validation of its interaction with E2 tagged ZFYVE27 (WT), N-terminus $(\Delta \mathrm{C})$, Cterminus $(\Delta N)$ as well as with ZFYVE27 lacking the core interaction region $(\triangle H R 3)$ by overexpression in NIH-3T3 cells and subsequent co-immunoprecipitation experiments. The cell lysates were subjected to immunoprecipitation with E2 tag antibody and the resulting immunoprecipitants were analyzed in immunoblot with c-Myc tag antibody (Co-IP: E2; WB: c-Myc). A portion of the cell lysates (input) was subjected to immunoblot with either c-Myc (WB: c$\mathrm{Myc}$ ) or E2 (WB: E2) tag antibodies to verify the protein expression of the indicated constructs. For mock experiments, the protein lysates from overexpressed cells were precipitated with non-specific $\mathrm{IgG}$ and subsequently analyzed by immunoblotting (as described above).

We next analyzed the ZFYVE27 self-interaction using co-localization experiments in mammalian cells. Overexpression of ZFYVE27 in both neuronal and nonneuronal cells induced the neurite formation (Fig. 3). Co-expression of both E2-ZFYVE27-WT and c-MycZFYVE27-WT in NIH-3T3 as well as HeLa cells showed the co-localization and indicated the profound neurite like extensions (Fig. 3A-C and D-F). In the neuronal NSC34 cells, overexpression of E2-ZFYVE27WT and c-Myc-ZFYVE27-WT also showed the colocalization primarily at the neuron growth cone and also in the cell body (Fig. 3G-I).

ZFYVE27 assembles into a homo-dimer/tetramerInterestingly, immunoblot analysis of protein lysates from cells overexpressing either E2-ZFYVE27-WT or c-Myc-ZFYVE27-WT showed a distinct band of size that corresponds to dimeric form of the protein in addition to the pronounced ZFYVE27 monomer (Fig.
4A). To validate the observed SDS-resistant dimeric form of ZFYVE27, we performed sucrose gradient centrifugation experiments with detergent solubilized cell lysates from NIH-3T3 cells overexpressing E2ZFYVE27-WT. Towards this, the cells were solubilized with $1 \%$ Big-CHAPS, subjected to sucrose gradient centrifugation and analyzed by SDS-PAGE. The oligomeric ZFYVE27 was visualized by immunoblotting with ZFYVE27 antibody. The immunoblot showed diffused pattern with the migration of E2-ZFYVE27-WT mainly in fractions corresponding to the molecular weight between dimer/tetramer forms of ZFYVE27 (Fig. 4B, C). Further analysis of immunoblot revealed that the distinct SDS-resistant dimers also have a similar migration profile of dimer/tetramer as the monomeric/SDS-sensitive form of ZFYVE27 (Fig. 4B, C).

ZFYVE27 is a peripheral membrane protein- To assess the membrane association properties of ZFYVE7, we used NSC34 cells, which express ZYFVE27 endogenously. The immunoblot analysis with ZFYVE27 antibody on sub-cellular fractions obtained from NSC34 cells indicated that ZFYVE27 is a membrane protein, as the monomeric ZFYVE27 was readily visible in membrane fraction (Fig. 5A). Interestingly, a band size corresponding to SDS-

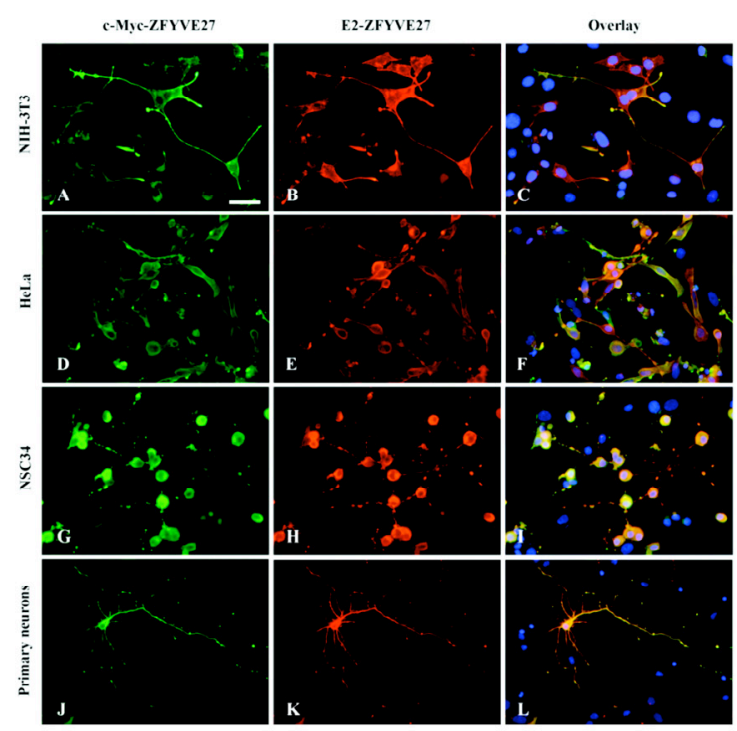

Figure 3. Co-localization of ZFYVE27 monomers in various cell lines. The human full-length ZFYVE27 fused with either c-Myc (cMyc-ZFYVE27-WT) or E2 (E2-ZFYVE27-WT) epitope tag were analyzed for the interaction in mammalian co-localization experiments. Overexpression of both the constructs in NIH-3T3, a fibroblast cell highlighted the formation of pronounced neurite like extensions (A, B) and showed strong co-localization of differentially tagged ZFYVE27 in neurite ends (C). Similar neurite like extensions and co-localization was observed in HeLa cells also (D-F). Overexpression of both ZFYVE27 tagged proteins in NSC34 indicated the neurite formation and co-localization of ZFYVE27 within neurites, these cells also showed swellings along the neurites (G-I), which is indicative of the accumulation of membrane organelles. Mouse primary hippocampal neurons overexpressing both ZFYVE27 tagged proteins showed extensive neurite formation and co-localization of ZFYVE27 monomers (J-L) and also showed neuronal swellings. Scale bars $-50 \mu \mathrm{m}(\mathrm{A}-\mathrm{L})$. 


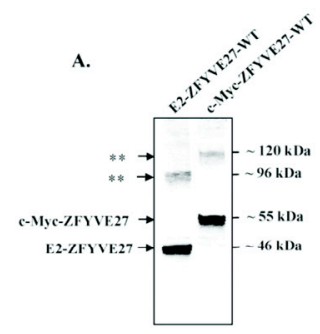

B.
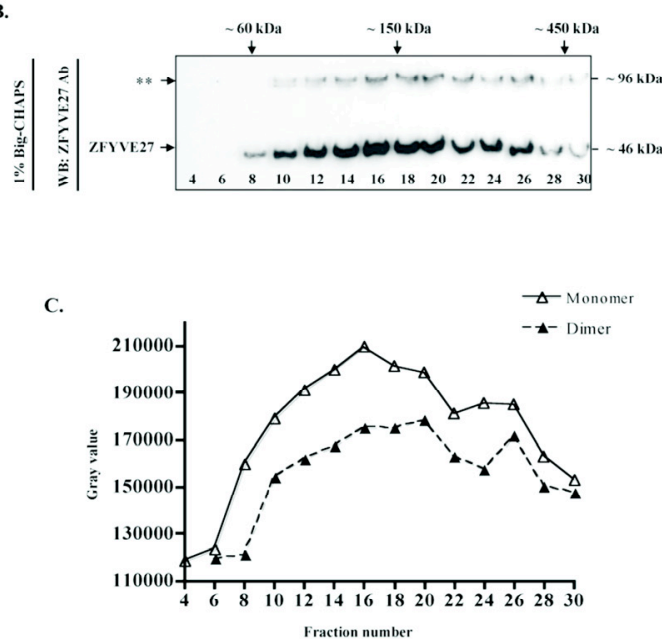

Figure 4. ZFYVE27 assembles into SDS-sensitive and-resistan dimer/tetramer. $(\Lambda)$ The protein lysates from NIH-3T3 cells overexpressing either E2-ZFYV27-WT or c-Myc-ZFYVE27-WT were subjected to immunoblot with ZFYVE27 specific antibody. The asterisks denote the formation of SDS-resistant dimers corresponding to E2-ZFYV27 ( 96 kDa) and c-Myc-ZFYVE27 ( $120 \mathrm{kDa}$ ). (B) The NSC34 cells were solubilized in 1\% Big-CHAPS and fractionated by $5-30 \%$ sucrose gradient centrifugation. The indicated resultant fractions were analyzed by immunoblot with ZFYVE27 antibody and the asterisks denote the formation of SDSresistant dimers. The mobility of molecular weight markers in the sucrose gradient are indicated on the top. (C) The immunoblot was used for the densitometry quantification in ImageJ program and the resulting mean gray values were plotted over the corresponding fractions. The density of the monomeric ZFYVE27 was represented in solid line with a peak in fractions corresponding to dimer/tetramer, whereas SDS-resistant dimers were indicated with the dashed line showing the similar pattern as observed for monomeric ZFYVE27.

resistant dimeric form of ZFYVE27 was visible in favor of our previous observations, but appeared exclusively in cytosolic fraction (Fig. 5A). In the next step, we treated the membrane fractions with Triton X-114, which dissociates the peripheral membrane proteins from membrane. Immunoblot analysis of the resultant aqueous and detergent phases after fractionation revealed that ZFYVE27 is a peripheral membrane protein (Fig. 5A).

To assess the membrane association properties of spastin, a known ZFYVE27 interacting protein, we transfected NSC34 cells with GFP-Myc-spastin (long isoform) and made sub-cellular fractionation. The immunoblot analysis with GFP antibody on sub-cellular fractions obtained from NSC34 cells overexpressing spastin indicated that it is also a membrane protein, as most of the protein was detected in the membrane fraction (Fig. 5B), however, a small amount of spastin was also detected in cytosolic fraction (Fig. 5B) Further, Triton X-114 phase separation of membrane fraction revealed that spastin is also a periphera membrane protein (Fig. 5B) as majority of the protein could be detected in aqueous phase, although small amount of spastin was still detected in detergent phase (Fig. 5B)

FYVE domain of ZFYVE27 has affinity towards al the phosphorylated derivatives of phosphatidylinosito lipids-The FYVE domain of several FYVE family proteins was shown to bind specifically to PtdIns3P (phosphatidylinositol 3-phosphate) lipid molecules $(22,35,36)$ and is mediated by conserved FYVE signature motifs like WXXD, RVC and R(R/K)HHCR motifs $(23,37)$. In silico analysis on the FYVE domain from ZFYVE27 revealed that though it has the conserved cystine residues to co-ordinate the zinc binding, it lacks the conserved FYVE signature motifs
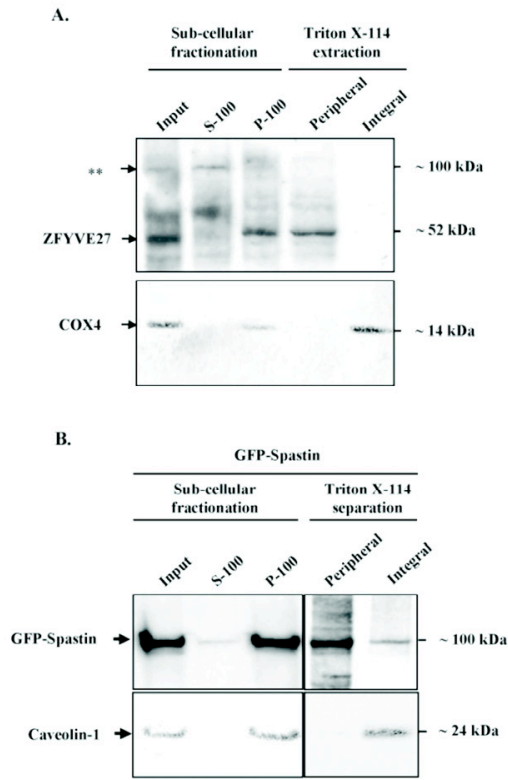

Figure 5. ZFYVE27 and spastin are peripheral membrane proteins. (A) Sub-cellular fractionation was performed on NSC34 cells and the resulting cytosolic fraction (S-100) and the membrane fraction (P-100) were analyzed by immunoblot with ZFYVE27 antibody. The asterisks denote the SDS-resistant dimers. The membrane fraction (P-100) was subjected to Triton X-114 phase separation and equal volumes of aqueous phase (peripheral) and detergent phase (integral) fractions were analyzed by immunoblotting. The blot was stripped and re-probed with COX4 antibody, a marker for the integral membrane protein. (B) The NSC34 cells overexpressing GFP-spastin were used for the subcellular fractionation. Equal volumes of the resulting cytosolic fraction (S-100) and the membrane fraction (P-100) in were analyzed together with a portion of the homogenate (input) by immunoblot with GFP antibody. The resulting membrane fraction (P-100) was subjected to Triton X-114 phase separation and equal volumes of aqueous phase (peripheral) and detergent phase (integral) fractions were subjected to immunoblotting with GFP antibody. The blots were stripped and re-probed with Caveolin-1 antibody, also a marker for the integral membrane protein. 
like WXXD, RVC and R(R/K)HHCR motifs (Fig. 6A). To assess the lipid binding properties of FYVE domain of ZFYVE27, we generated a recombinant HisZFYVE2 $7^{150-404}$ fusion protein and assayed its ability to bind to various phospholipids (Fig. 6B). The recombinant ZFYVE27 showed affinity exclusively towards all the phosphorylated derivatives of phosphatidylinositol, with high affinity towards PtdIns(5)P and PtdIns $(3,4,5) \mathrm{P}_{3}$ and moderate binding to PtdIns(3)P, PtdIns(4)P, PtdIns(3,4) $\mathrm{P}_{2}, \operatorname{PtdIns}(3,5) \mathrm{P}_{2}$, PtdIns(4,5) $\mathrm{P}_{2}$ (Fig. 6B). The FYVE domain of ZFYVE27 did not show any affinity towards other analyzed lipids (Fig. 6B) and showed no affinity towards sphingolipids (data not shown).

A.

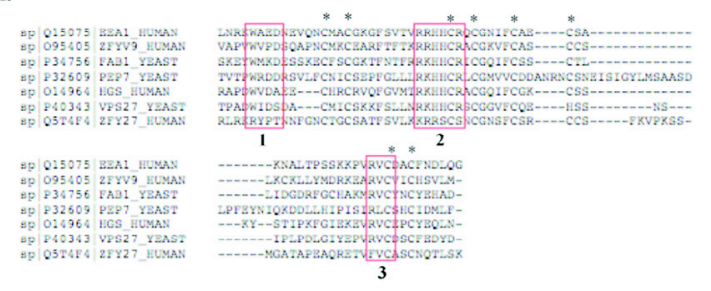

B.

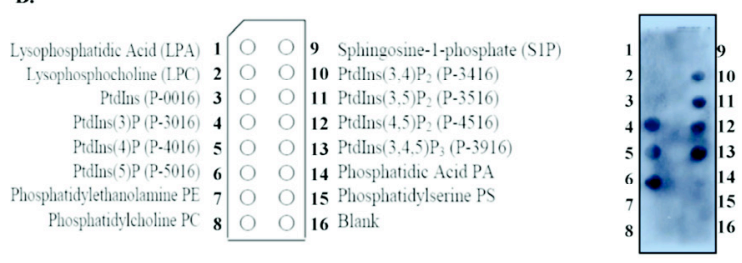

Figure 6. The FYVE domain and the membrane binding properties of ZFYVE27. (A) Alignment of the FYVE domain of ZFYVE27 (ZFY27) with other FYVE family proteins; EEA1, ZFYV9, HGS/HRS and yeast FAB1, PEP7, VPS27. The red boxes showing WXXD motif (1), R(R/K)HHCR motif (2) and RVC motif (3) are highlighted. The conserved cystine residues were marked with asterisks. (B) The lipid-protein overlay assay with recombinant ZFYVE27 protein. Schematic diagram of the nitrocellulose membrane spotted (100 picomole) with immobilized phospholipids: PIP Strip (left panel). The PIP strip was incubated with $\sim 500 \mathrm{ng} / \mathrm{mL}$ of the purified recombinant His- $\triangle \mathrm{N}-$ ZFYVE27 and the bound protein was detected with antibodies against His-tag and subsequent chemiluminescence detection.

Core interaction region (HR3) is indispensable for neurite extension by ZFYVE27- Finally, owing to the fact that ZFYVE27 can induce the neurite formation in both neuronal and non-neuronal cells (15), we sought to determine the function/relevance of oligomeric ZFYVE27 in this process. As expected, overexpression of E2-ZFYVE27-WT promoted neurite like extensions in both non-neuronal cells (Fig. 7A, B) and in neuronal cells (Fig. 7C, D). Surprisingly, overexpression of E2ZFYVE27- $\triangle \mathrm{HR} 3 \mathrm{did}$ not promote the neurite extension in all the analyzed cell lines (Fig. 7E-H) and showed accumulation of the protein in tubular structures with swelling of cell soma and increase in the cell volume. In the next step, we co-expressed E2-ZFYVE27- $\Delta$ HR3 together with c-Myc-ZFYVE27-WT to assess the effect of mutant protein on the wild type ZFYVE27 function as the interaction studies suggested they form oligomers. Consistent with our co-immunoprecipitation data, E2-ZFYVE27- $\triangle$ HR3 showed the co-localization with wild type protein and more interestingly, the E2ZFYVE27- $\triangle$ HR3 inhibited the ability of c-MycZFYVE27-WT to promote neurite-like/neurite structures in both non-neuronal (Fig. 7I-N) and neuronal cells (Fig. 7O-T) respectively. The observed neurite inhibition by E2-ZFYVE27- $\triangle \mathrm{HR} 3$ was much more pronounced in mouse primary neurons as these cells showed the retarded growth of neurites (Fig. R-T).

\section{DISCUSSION}

ZFYVE27 was initially identified as a spastin binding protein (12). Recent progress in understanding the function of ZFYVE27 clearly demonstrates its pivotal role in neurite extension through the membrane recycling system $(15,16,18)$. Recently, Yu et al., (2008) (14) could demonstrate that spastin also functions in neuritogenesis (neurite branch formation), thus highlighting the common physiological function for both spastin and ZFYVE27 in a molecular process relevant to HSP.

To gain further insights into the molecular mechanism of ZFYVE27 in neuritogenesis and in HSP, we performed a yeast-two hybrid (Y2H) screen and identified ZFYVE27 to interact with itself. Coimmunoprecipitation and co-localization experiments in mammalian cells further confirmed the observed ZFYVE27 self-interaction. Further, we mapped the core interaction region to HR3 motif of ZFYVE27 and the deletion of this region did not abolish the interaction with its wild type counterpart in cell culture experiments. Interestingly, deletion of FYVE domain and HR3 from ZFYVE27 abolished the interaction in direct-Y2H experiments. These observations indicate that there could be another functional/structural regions through which ZFYVE27 self-association could be stabilized mainly via coiled-coil region together with FYVE domain of ZFYVE27 or through N-terminus, as this region showed a weak interaction in our direct-Y2H studies. The coiled-coil region of another FYVE family protein EEA1 was shown to be important for homodimerization and juxtaposes two FYVE domains allowing simultaneous interaction with two PtdIns3P head groups (23). The common notion that FYVE domains may influence the protein's ability to associate with endosomal membranes via their ability to form oligomers as observed for EEA1 (25), SARA (26) and Hrs (38) further supports the observed oligomeric form of ZFYVE27.

To decipher the oligomeric nature of ZFYVE27, we employed sucrose gradient centrifugation method and could show that ZFYVE27 mainly oligomerizes into dimer/tetramer. Notably, the immunoblot analysis showed the higher molecular weight band, whose size corresponds to dimer in conventional SDS-PAGE suggesting that it might form SDS resistant dimers. 

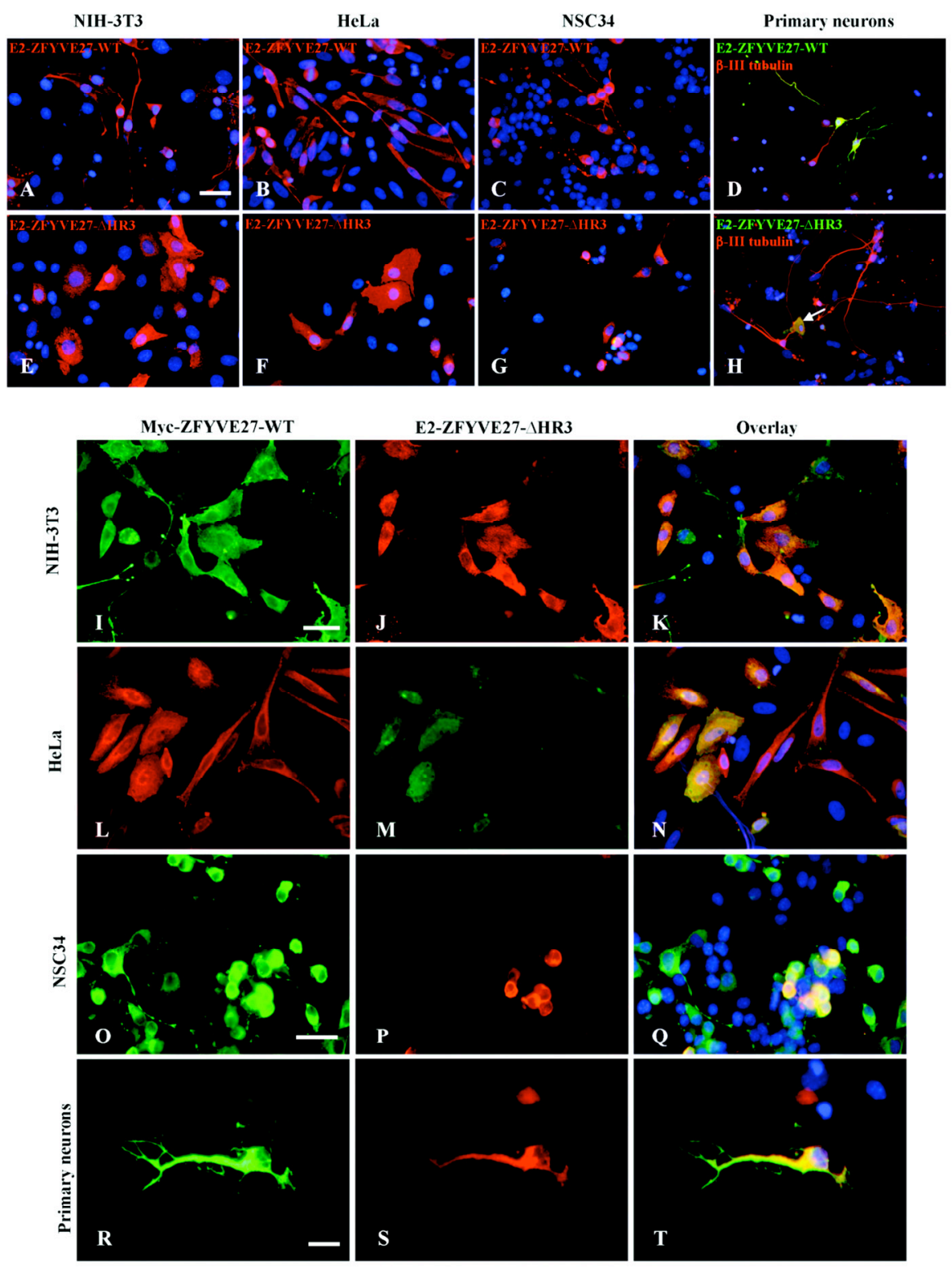

Figure 7. ZFYVE27- $\triangle$ HR3 deters the neurite extension formation by wild type ZFYVE27: Overexpression of E2-ZFYVE27-WT showed the neurite like/neurie extension in all the analyzed cell lines (A-D), whereas overexpression of E2-ZFYVE27-AHR3 (truncated ZFYVE27) failed to induce the neurite extension in the same set of cell lines and caused swelling of the cytoplasm (E-H). Primary neurons were stained with neuronal marker $\beta$-III tubulin $(\mathrm{B}, \mathrm{D})$ and neuron overexpressing E2-ZFYVE27- $\Delta$ HR3 showed no neurites (arrow). Coexpression of both the wild type and truncated ZFYVE27 in both non-neuronal (I-N) and in neuronal cells (O-T) showed the colocalization of wild type with truncated ZFYVE27. The cells overexpressing wild type protein alone showed the neurite extension (I, L, O and $\mathrm{R}$ ) whereas cell co-expressing wild type as well as ZFYVE27- $\mathrm{HR} 3$ failed to produce neurite extension rather caused swelling of

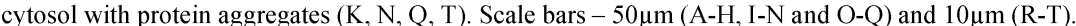

This feature of forming SDS resistant oligomers has been observed for many other proteins especially, EEA1, a well characterized FYVE family protein (25). Interestingly, the observed SDS resistant oligomers appeared exclusively in the cytosolic fraction, but not in membrane fraction. Further, our sub-cellular fractionation and Triton X-114 phase separation data revealed that ZFYVE27 is a peripheral membrane protein in contrast to the in silico prediction of being an integral membrane protein as it contains three putative $\mathrm{HR} /$ transmembrane (TM) domains. To date all the characterized FYVE family proteins are expressed as soluble proteins and targeted to the membrane via their FYVE domain. While deletion or mutagenesis studies in the conserved regions of FYVE domain showed a dramatic affect on the avidity and the membrane binding properties with diffused cytosolic pattern $(23,26,39,40)$. RBD11 motif of ZFYVE27 was shown to be responsible for its interaction with Rab11 (15) and this motif overlaps with the HR1. Interestingly, our observed self-interaction of ZFYVE27 is mainly mediated by HR3, thus suggesting the role for 
hydrophobic regions of ZFYVE27 in protein-protein interactions. Spastin has been shown to interact with ZFYVE27 through its N-terminal part, which contains a putative TM and microtubule interacting and trafficking (MIT) domains (12). Since, the MIT domain of spastin has been implicated in interaction with microtubules, it is interesting to address the role of TM domain in membrane localization of spastin. Our membrane association studies on full-length spastin showed that spastin is also a peripheral membrane protein suggesting that the TM domain/hydrophobic region of spastin might mediate its interaction with ZFYVE27. Recently, ZFYVE27 was shown to interact with VAP-A via FFAT motif (18), more importantly, VAP proteins are known to mediate the recruitment of FFAT motif containing proteins to endoplasmic reticulum membrane (41), further strengthening that ZFYVE27 is a cytosolic protein and translocates into membrane via interaction with VAP.

The classical FYVE domain possess three signature motifs: an N-terminal WXXD motif, a basic $\mathrm{R}(\mathrm{R} / \mathrm{K}) \mathrm{HHCR}$ motif and a C-terminal RVC motif, which specifically recognize and binds to PtdIns3P $(22,24,35)$. Our in silico analysis revealed that FYVE finger motif of ZFYVE27 lacks conserved WXXD, $\mathrm{R}(\mathrm{R} / \mathrm{K}) \mathrm{HHCR}$ and RVC motifs. When, we analyzed the membrane binding properties of ZFYVE27 using lipidprotein overlay assay with recombinant HisZFYVE2 $7^{150-404}$ fusion protein, the FYVE domain of ZFYVE27 showed affinity towards all the phosphorylated derivatives of PtdIns (Phospatidylinositol), but not to any other analyzed lipid molecules. Collectively, these observations make ZFYVE27 distinct from other FYVE family proteins which bind specifically to PtdIns3P. Although the major distinguishing feature of a typical FYVE domain is specific recognition of PtdIns3P, binding and the membrane anchoring to the acidic lipids other than PtdIns3P is found to be mediated by multivalent mechanisms and involves non-specific electrostatic interactions (42-44), insertion of hydrophobic regions into the lipid bilayers $(37,43,45)$ and in some cases dimerization $(23,25,26)$. These observations indicate that ZFYVE27 might contain novel functional/structural regions, which can facilitate its recruitment to the membrane compartments other than PtdIns3P containing early endosomes. This feature might allow ZFYVE27 to participate in both secretary as well as endocytic pathways as a peripheral membrane protein or might be involved in the trafficking of all the PtdIns lipid molecules, thus providing the membrane necessary for neurite extension as a 'master molecule'.

Owing to the fact that ZFYVE27 can induce the neurite formation in both neuronal and non-neuronal cells (15), we sought to investigate the functional relevance of oligomeric ZFYVE27 in neurite extension. Overexpression of wild-type ZFYVE27 induced the neurite formation in all the analyzed cell lines. Interestingly, expression of $\triangle \mathrm{HR} 3$ mutant (deletion of core interaction region) inhibited the neurite extension in the cells and showed aggregation of protein in tubular structures with swelling of the cytoplasm. Coexpression of $\triangle \mathrm{HR} 3$ mutant together with wild-type ZFYVE27 showed the inhibition of neurite extension by wild-type ZFYVE27, suggesting the dominant negative effect of $\triangle H R 3$ mutant. ZFYVE27 is known to interact with spastin (12) and with VAP (18), and mutations in spastin and VAP are associated with HSP (46) and ALS (amyotrophic lateral sclerosis) disorders (47) respectively. Interestingly, overexpression of mutant spastin has shown to redistribute the wild-type spastin to filamentous tubules in a dominant negative fashion $(1,33)$. Similarly, expression of ALS associated mutant form of VAP-B has been shown to form tubular aggregates and moreover, mutant VAPB traps the wildtype VAP proteins in aggregates and in turn impairs the function (48) indicating the dominant negative mechanism of mutant VAP-B. Taken together, it appears that Spastin, ZFYVE27, VAP and Rab11 proteins might together constitute a system for the regulation of vesicular transport or lipid trafficking in neurons and impairment of this system may be responsible for the pathogenesis of neurodegenerative diseases such as HSP and ALS. Generation of "loss of function" animal model for ZFYVE27 will provide further insights into the molecular function of ZFYVE27 in neuritogenesis and role in pathogenesis of HSP.

Acknowledgments- We thank M. Czyzewska and H. Hühn for their excellent technical assistance. This work was funded by Deutsche Forschungsgemeinschaft (MA 3344/2-1) Grant to A. U. M.

\section{REFERNCES}

1. Errico, A., Ballabio, A., and Rugarli, E. I. (2002) Hum $\mathrm{Mol}$ Genet 11(2), 153-163

2. Fonknechten, N., Mavel, D., Byrne, P., Davoine, C. S., Cruaud, C., Bonsch, D., Samson, D., Coutinho, P., Hutchinson, M., McMonagle, P., Burgunder, J. M., Tartaglione, A., Heinzlef, O., Feki, I., Deufel, T., Parfrey, N., Brice, A., Fontaine, B., Prud'homme, J. F., Weissenbach, J., Durr, A., and Hazan, J. (2000) Hum Mol Genet 9(4), 637-644

3. Hazan, J., Fonknechten, N., Mavel, D., Paternotte, C., Samson, D., Artiguenave, F., Davoine, C. S., Cruaud, C., Durr, A., Wincker, P., Brottier, P., Cattolico, L., Barbe, V., Burgunder, J. M., Prud'homme, J. F., Brice, A., Fontaine, B., Heilig, B., and Weissenbach, J. (1999) Nat Genet 23(3), 296-303

4. McDermott, C. J., Burness, C. E., Kirby, J., Cox, L. E., Rao, D. G., Hewamadduma, C., Sharrack, B., Hadjivassiliou, M., Chinnery, P. F., Dalton, A., and Shaw, P. J. (2006) Neurology 67(1), 45-51

5. Patrono, C., Scarano, V., Cricchi, F., Melone, M. A., Chiriaco, M., Napolitano, A., Malandrini, A., De Michele, G., Petrozzi, L., Giraldi, C., Santoro, L., Servidei, S., Casali, C., Filla, A., and Santorelli, F. M. (2005) Hum Mutat 25(5), 506

6. Roll-Mecak, A., and Vale, R. D. (2005) Curr Biol 15(7), 650655

7. Sauter, S., Miterski, B., Klimpe, S., Bonsch, D., Schols, L., Visbeck, A., Papke, T., Hopf, H. C., Engel, W., Deufel, T., Epplen, J. T., and Neesen, J. (2002) Hum Mutat 20(2), 127-132

8. Shoukier, M., Neesen, J., Sauter, S. M., Argyriou, L. Doerwald, N., Pantakani, D. V., and Mannan, A. U. (2009) Eur J Hum Genet 17(2), 187-194

9. Errico, A., Claudiani, P., D'Addio, M., and Rugarli, E. I. (2004) Hum Mol Genet 13(18), 2121-2132 
10. Evans, K., Keller, C., Pavur, K., Glasgow, K., Conn, B., and Lauring, B. (2006) Proc Natl Acad Sci U S A 103(28), 1066610671

11. Mannan, A. U., Boehm, J., Sauter, S. M., Rauber, A., Byrne, P. C., Neesen, J., and Engel, W. (2006) Neurogenetics 7(2), 93103

12. Mannan, A. U., Krawen, P., Sauter, S. M., Boehm, J., Chronowska, A., Paulus, W., Neesen, J., and Engel, W. (2006) Am J Hum Genet 79(2), 351-357

13. Reid, E., Connell, J., Edwards, T. L., Duley, S., Brown, S. E., and Sanderson, C. M. (2005) Hum Mol Genet 14(1), 19-38

14. Yu, W., Qiang, L., Solowska, J. M., Karabay, A., Korulu, S., and Baas, P. W. (2008) Mol Biol Cell 19(4), 1485-1498

15. Shirane, M., and Nakayama, K. I. (2006) Science 314(5800), 818-821

16. Shirane, M., Ogawa, M., Motoyama, J., and Nakayama, K. I. (2008) Genes Cells 13(6), 635-651

17. Hamilton, G. S., and Steiner, J. P. (1998) J Med Chem 41(26), 5119-5143

18. Saita, S., Shirane, M., Natume, T., Iemura, S., and Nakayama, K. I. (2009) J Biol Chem 284(20), 13766-13777

19. Hayakawa, A., Hayes, S., Leonard, D., Lambright, D., and Corvera, S. (2007) Biochem Soc Symp (74), 95-105

20. Misra, S., and Hurley, J. H. (1999) Cell 97(5), 657-666

21. Stenmark, H., Aasland, R., Toh, B. H., and D'Arrigo, A. (1996) $J$ Biol Chem 271(39), 24048-24054

22. Burd, C. G., and Emr, S. D. (1998) Mol Cell 2(1), 157-162

23. Dumas, J. J., Merithew, E., Sudharshan, E., Rajamani, D., Hayes, S., Lawe, D., Corvera, S., and Lambright, D. G. (2001) Mol Cell 8(5), 947-958

24. Kutateladze, T. G. (2006) Biochim Biophys Acta 1761(8), 868877

25. Callaghan, J., Simonsen, A., Gaullier, J. M., Toh, B. H., and Stenmark, H. (1999) Biochem J 338 (Pt 2), 539-543

26. Hayakawa, A., Hayes, S. J., Lawe, D. C., Sudharshan, E., Tuft, R., Fogarty, K., Lambright, D., and Corvera, S. (2004) J Biol Chem 279(7), 5958-5966

27. Jayaraman, L., and Massague, J. (2000) J Biol Chem 275(52), 40710-40717

28. Mao, Y., Nickitenko, A., Duan, X., Lloyd, T. E., Wu, M. N., Bellen, H., and Quiocho, F. A. (2000) Cell 100(4), 447-456

29. Gietz, R. D., Schiestl, R. H., Willems, A. R., and Woods, R. A. (1995) Yeast 11(4), 355-360

30. Rupp, R. A., Snider, L., and Weintraub, H. (1994) Genes Dev 8(11), 1311-1323

31. Cashman, N. R., Durham, H. D., Blusztajn, J. K., Oda, K., Tabira, T., Shaw, I. T., Dahrouge, S., and Antel, J. P. (1992) Dev Dyn 194(3), 209-221

32. Durham, H. D., Dahrouge, S., and Cashman, N. R. (1993) Neurotoxicology 14(4), 387-395

33. Pantakani, D. V., Swapna, L. S., Srinivasan, N., and Mannan, A. U. (2008) J Neurochem 106(2), 613-624

34. Bordier, C. (1981) J Biol Chem 256(4), 1604-1607

35. Gaullier, J. M., Simonsen, A., D'Arrigo, A., Bremnes, B., Stenmark, H., and Aasland, R. (1998) Nature 394(6692), 432433

36. Patki, V., Lawe, D. C., Corvera, S., Virbasius, J. V., and Chawla, A. (1998) Nature 394(6692), 433-434

37. Kutateladze, T., and Overduin, M. (2001) Science 291(5509), 1793-1796

38. Pullan, L., Mullapudi, S., Huang, Z., Baldwin, P. R., Chin, C., Sun, W., Tsujimoto, S., Kolodziej, S. J., Stoops, J. K., Lee, J. C., Waxham, M. N., Bean, A. J., and Penczek, P. A. (2006) Structure 14(4), 661-671

39. Coumailleau, F., Das, V., Alcover, A., Raposo, G., Vandormael-Pournin, S., Le Bras, S., Baldacci, P., DautryVarsat, A., Babinet, C., and Cohen-Tannoudji, M. (2004) Mol Biol Cell 15(10), 4444-4456

40. Gaullier, J. M., Ronning, E., Gillooly, D. J., and Stenmark, H. (2000) J Biol Chem 275(32), 24595-24600

41. Loewen, C. J., Roy, A., and Levine, T. P. (2003) Embo J 22(9), 2025-2035

42. Diraviyam, K., Stahelin, R. V., Cho, W., and Murray, D. (2003) J Mol Biol 328(3), 721-736
43. Kutateladze, T. G., Capelluto, D. G., Ferguson, C. G., Cheever, M. L., Kutateladze, A. G., Prestwich, G. D., and Overduin, M. (2004) J Biol Chem 279(4), 3050-3057

44. Stahelin, R. V., Long, F., Diraviyam, K., Bruzik, K. S., Murray, D., and Cho, W. (2002) J Biol Chem 277(29), 26379-26388

45. Blatner, N. R., Stahelin, R. V., Diraviyam, K., Hawkins, P. T., Hong, W., Murray, D., and Cho, W. (2004) J Biol Chem 279(51), 53818-53827

46. Salinas, S., Carazo-Salas, R. E., Proukakis, C., Schiavo, G., and Warner, T. T. (2007) J Neurosci Res 85(12), 2778-2782

47. Nishimura, A. L., Mitne-Neto, M., Silva, H. C., Richieri-Costa, A., Middleton, S., Cascio, D., Kok, F., Oliveira, J. R., Gillingwater, T., Webb, J., Skehel, P., and Zatz, M. (2004) Am J Hum Genet 75(5), 822-831

48. Teuling, E., Ahmed, S., Haasdijk, E., Demmers, J., Steinmetz, M. O., Akhmanova, A., Jaarsma, D., and Hoogenraad, C. C. (2007) J Neurosci 27(36), 9801-9815 


\section{Discussion}

Mutations in the SPG4/SPAST gene are the most common cause for autosomal dominant hereditary spastic paraplegia (AD-HSP) and accounts for up to $40 \%$ of all ADHSP cases (Hazan et al., 1999; Fonknechten et al., 2000; Hentati et al., 2000; Lindsey et al., 2000; Sauter et al., 2002; Patrono et al., 2005; McDermott et al., 2006). In the present study, we demonstrate that the SPAST protein product, spastin, function as a hexamer. Moreover, we refined/expanded the mutational spectrum of SPAST in a large HSP cohort and evaluated the effects of these mutations on the oligomeric spastin function. In addition, we characterized the molecular and biochemical properties of ZFYVE27, a spastin interacting protein, which is involved in HSP. Furthermore, generation of loss of function and gain of function mouse models for ZFYVE27 were endeavored.

\subsection{Spastin assembles into hexamer for its microtubule severing activity}

Spastin belongs to the AAA (ATPases Associated with various cellular Activities) family of proteins, which are characterized by the presence of AAA domain, often at the carboxy-terminal region of the protein. Sequence homology analysis indicated that spastin is closely related to sub-group 7 of AAA family of proteins. Notable members of this group are P60-katanin, a well characterized microtubule (MT) severing enzyme and VPS4/SKD1, which is involved in vacuolar protein sorting and endosomal trafficking (Frickey and Lupas, 2004). Recent advances in understanding the function of spastin has clearly demonstrated that spastin is also a MT severing enzyme (Errico et al., 2002; Evans et al., 2005; Roll-Mecak and Vale, 2005). Majority of the AAA proteins renders their function by forming oligomeric structures, mostly a hexamer (6 sub-units) or do- decamer (12 sub-units). Based on the above facts, it is likely that spastin might also form an oligomer to elicit its function.

In the present study (Pantakani et al., 2008a), we attempted to determine the oligomeric form of spastin by employing chemical cross-linking and gel filtration chromatography techniques and could show that spastin (short isoform) assembles into a hexamer. Recently, White and colleagues (2007) also reported that spastin could assemble into hexamer by using truncated form of spastin $(\Delta 1-227)$ with a Walker B motif mutation E442Q, however, they failed to show the oligomeric form for the wild type spastin. The discrepancy between our study and those of White et al., (2007) could be 
due to the usage of full-length spastin (our study) as compared to truncated spastin $(\Delta 1$ 227) used by White et al., (2007). The full-length spastin is a true representative of endogenous protein and perhaps $\mathrm{N}$-terminal region of spastin might act as a linker motif and stabilize the hexameric form of spastin, as also shown for another AAA protein, p97/VCP (Wang et al., 2003). Our data was further supported by a recent study (RollMecak and Vale, 2008), where it was shown that the wild type spastin (from Caenorhabditis elegans) can also assemble into hexamer and they identified a conserved linker motif between $\mathrm{N}$-terminus and AAA domain of spastin, which acts as a hexamer stabilizing region.

The structural basis for the spastin's MT severing activity came from the recent studies including from our group (Pantakani et al., 2008a) and demonstrated that the spastin hexameric ring has a central pore and two pore loops projected into the pore cavity as depicted in figure 4.1A (White et al., 2007; Roll-Mecak and Vale, 2008). The pore loop 1 lies near the orifice of the pore and mutations in this region abolished the MT severing but preserved the MT binding of the enzyme, whereas pore loop 2 resides deeper in the pore and mutations in this region severely inhibits the MT severing activity (White et al., 2007; Roll-Mecak and Vale, 2008). Collectively from these studies it can be postulated that spastin docks onto MTs as a hexamer in ATP dependent manner, placing the positively charged $\mathrm{N}$-terminal pore of spastin in contact with the negatively charged $\mathrm{C}$ terminal amino acids of tubulin tail and pulls into the pore cavity as illustrated in figure 4.1B, C. These observations led to the hypothesis that spastin just grip the C-terminal tubulin tail and exert mechanical force that destabilize tubulin polymer locally leading to breakdown of the MT polymer.

To gain further insights into the oligomeric form of spastin, we generated a structural model of the AAA domain of spastin using templates of spastin structural homologues, namely VPS4B (Scott et al., 2005) and p97/VCP (Dreveny et al., 2004). Next, we used our spastin model as a framework to categorize the identified spastin missense mutations in HSP patients into four different functional groups namely active site, pore loop, monomermonomer interaction and unknown structural groups based on the known structural/functional regions of the AAA domain. Interestingly, several of these functional/structural group mutations have been already validated for their deleterious effect in various cellular studies (Errico et al., 2002; McDermott et al., 2003; 
Evans et al., 2005; Sanderson et al., 2006; Pantakani et al., 2008a). In future, the spastin functional/structural categorization will enable us to classify any newly identified AAA domain mutation as a disease causing mutation with greater confidence.
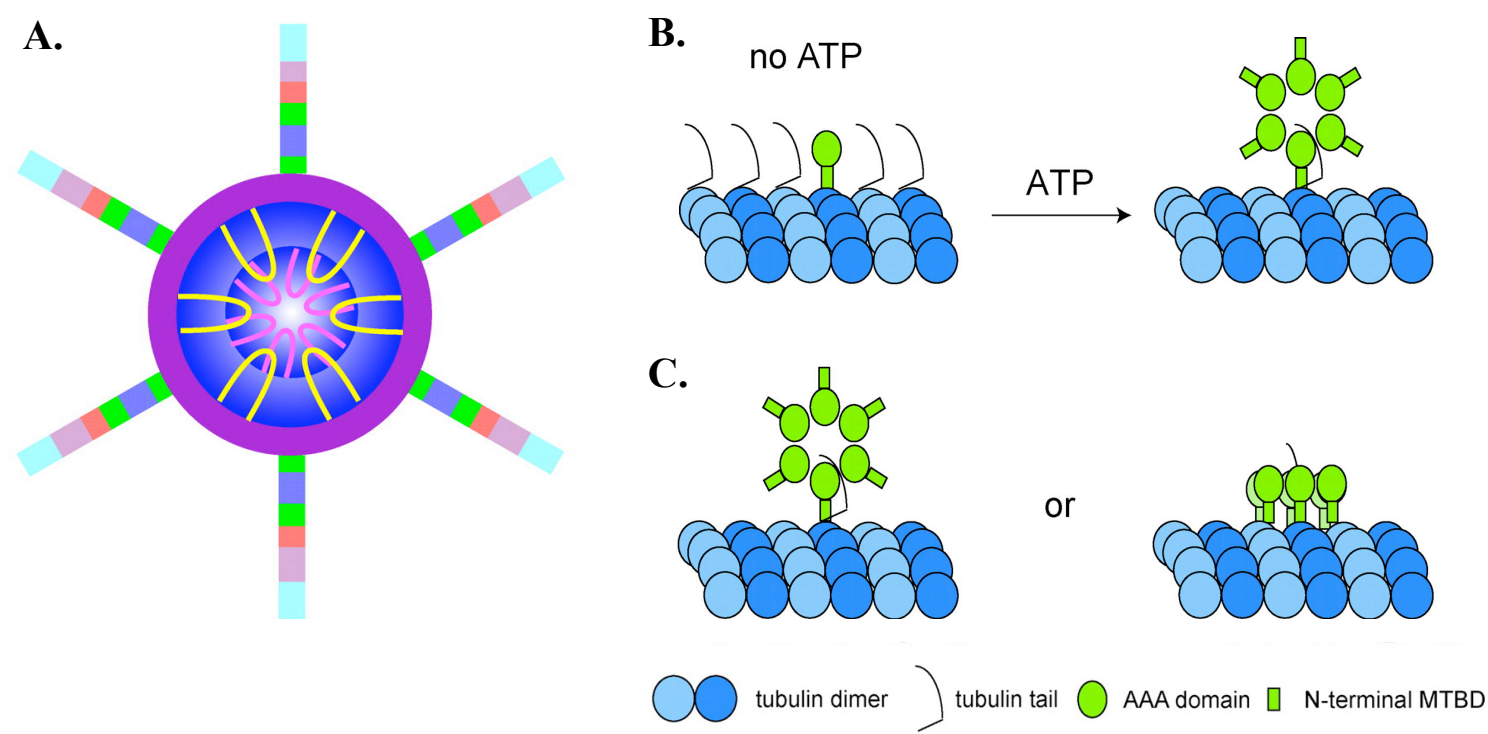

Figure 4.1. Structural basis for the hexameric spastin pore loops formation and model for microtubule severing by spastin. (A) Schematic depiction of hexameric spastin ring formation (purple). Each monomer of spastin contributes to two pore loops: pore loop 1 (yellow) lies near the pore surface and pore loop 2 (pink) resides deeper in the pore. N-terminal regions are indicated in six radiating arms. (B) Unassembled spastin (green) can bind to microtubule by an ATP independent manner. Binding of ATP to spastin results in assembly of the hexamer and pore formation. The hexameric spastin AAA core recognizes the C-terminal tubulin tail (not all tubulin tails are drawn for clarity). (C) Two possible orientations of spastin hexamer on tubulin polymer, leading to the pulling of tubulin tail into the pore cavity and subsequent breakdown of local tubulin polymer (adapted from White et al., 2007).

\subsection{Expansion of mutational spectrum and evaluating the effects of spastin mutations in HSP}

Spastin is most frequently mutated in AD-HSP, till to date over 300 different mutations including missense, nonsense, splice site and insertions/deletions have been reported all along the primary structure of spastin (with the exception of the alternatively spliced exon 4). Recently, $S P A S T$ gene rearrangements, in particular exon deletions, were also shown to be a common cause for the SPAST-HSP (Beetz et al., 2006; Depienne et al., 2007). The wide spectrum of spastin mutations suggests that haploinsufficiency as the underlying cause for the disease. Apart from this view, several studies including from our 
group (Pantakani et al., 2008a) also proposed that spastin missense mutations might act in a dominant negative manner. Notably, overexpression of spastin mutants-E442Q (Pantakani et al., 2008a) and K338R (Errico et al., 2002) redistributed the wild type spastin expression from vesicles to filamentous structures indicating the dominant negative function of mutant protein and causing the redistribution/hampering of the wild-type counterpart function. In support of this notion, expression of truncated spastin peptide (comprising long isoform of spastin) in neurons inhibited the fast axonal transport, which further highlights the 'gain of function' mechanism (Solowska et al., 2008). Moreover, the 'threshold effect model' is also relevant at least for a sub-set of SPAST mutations, where a direct correlation between functional level of spastin and early onset of HSP phenotype has been described (Svenson et al., 2001b; Svenson et al., 2004).

In support of threshold effect model, in a case report study (Pantakani et al., 2008b), we have identified compound heterozygous sequence variants [c.131C $>\mathrm{T}$ (S44L) and c. $1687 \mathrm{G}>\mathrm{A}(\mathrm{E} 563 \mathrm{~K})]$ of $S P A S T$ causing a severe form of HSP in a patient. The $\mathrm{S} 44 \mathrm{~L}$ polymorphism is known to act as a modifier of HSP phenotype (Lindsey et al., 2000; Chinnery et al., 2004; Svenson et al., 2004; McDermott et al., 2006). In our case study, the mutation c. $1687 \mathrm{G}>\mathrm{A}$ (alone) appears to be non- pathogenic as family members carrying solely the c. $1687 \mathrm{G}>$ A allele are asymptomatic for HSP, however together with S44L causes a severe phenotype. Further, we confirmed that c. $1687 \mathrm{G}>\mathrm{A}$ is a leaky splice site mutation and leads to skipping of exon 15 of spastin. Our observation that the association of S44L polymorphism with non-disease causing and leaky splice site mutation further supports the reported modulatory role for S44L in the severity of HSP phenotype in the absence or presence of disease causing mutations (Svenson et al., 2004). Our findings further strengthen the 'threshold effect model' for spastin and suggest that there is a direct correlation between functional level of spastin and symptom severity in the spastin-linked HSP.

Furthermore, in a recent study (Shoukier et al., 2009), we made attempts to expand the mutational spectrum of SPAST in a large HSP cohort. Towards this end, we screened for mutations in the SPAST gene in a cohort of 200 HSP patients and could identify 47 different mutations. Our SPAST mutation detection rate of $28.5 \%$ was in accordance with the previous mutation detection rates (15-44\%) for SPAST in other ethnic HSP populations (Fonknechten et al., 2000; Lindsey et al., 2000; Patrono et al., 2005; Crippa et al., 2006). 
Interestingly, out of 26 novel point mutations, 22 mutations were located in the AAA domain of spastin. Besides few exceptions, almost all the missense mutations reported until now in spastin are located in the AAA domain of spastin. Moreover, when we studied the distribution of all the reported spastin mutations over the structural architecture of spastin, we identified AAA domain as the primary cluster/hot spot region for mutations. We also identified three other secondary clusters/hotspot regions in MIT (Microtubule Interacting and Trafficking) domain, MTBD (Microtubule Binding Domain) and an un-identified functional N-terminal region (228-269 residues). Overall, it appears that different functional domains/regions of spastin are prime targets for mutations thus highlighting the need to set these regions as priority in the molecular diagnosis of SPAST-HSP. Moreover, categorization of identified missense mutations into two different age groups, early age onset ( $\leq 35$ years) and late age onset ( $>35$ years), showed a tentative genotype-phenotype correlation and suggests that in case of missense mutations the onset of phenotype is earlier.

\subsection{Elucidating the molecular function of ZFYVE27, a spastin interacting protein}

Spastin has been shown to interact with several vesicular trafficking proteins namely; RTN1, Atlastin, CHMP1B and ZFYVE27/Protrudin (Reid et al., 2005; Evans et al., 2006; Mannan et al., 2006a, b). In particular, ZFYVE27 was identified as a novel spastin interacting protein by our group (Mannan et al., 2006b) and reported as a causative gene for HSP. ZFYVE27 was shown to be a key regulator in promoting the neurite extension by directed membrane trafficking via recycling endosomes through its association with Rab 11 (Shirane and Nakayama, 2006). The interaction of ZFYVE27 with spastin and Rab 11 indicates that these proteins might together be involved in regulation of the neuron specific vesicle/membrane trafficking and impairment of such neuronal cargo transport system might lead to neurodegeneration. To dissect the role of ZFYVE27 and the significance of its interaction with spastin in the context of HSP pathomechanism, we decided to characterize the molecular function of ZFYVE27.

In the current study (manuscript in submission stage: Pantakani and Mannan, 2009), we performed yeast-two hybrid (Y2H) screen using human ZFYVE27 (isoform 4) as a bait protein to identify its interacting proteins. Interestingly, our $\mathrm{Y} 2 \mathrm{H}$ screen revealed that ZFYVE27 interacts with itself. The common notion that FYVE domains may influence the 
protein's ability to associate with endosomal membranes via their ability to form oligomers as observed for EEA1 (Fig. 4.2) (Callaghan et al., 1999), SARA (Hayakawa et al., 2004) and Hrs (Pullan et al., 2006) convinced us to explore the oligomeric nature and biochemical properties of ZFYVE27.

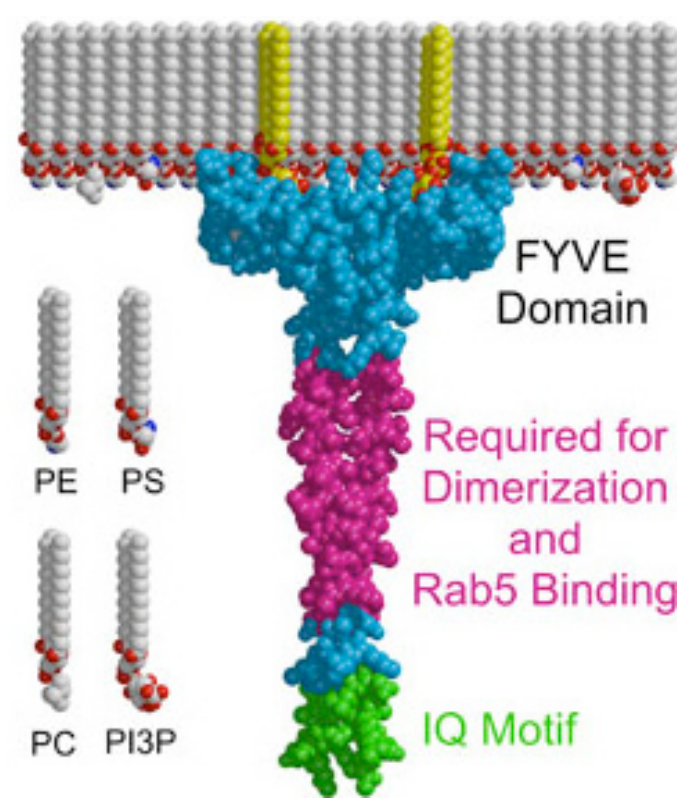

Figure 4.2. Model for multivalent membrane binding by FYVE domain of homodimeric EEA1. Membrane binding orientation of homodimeric EEA1, which specifically recognizes and simultaneously binds to two PtdIns(3)P (Phosphatidylinositol-3-phosphate) head groups of membrane by homodimeric FYVE domains (indigo). The region required for dimerization and interaction with Rab5 (magenta) and putative calmodulin binding IQ motif (green) are highlighted. The membrane leaflet was shown with different lipid molecules: PE - Phosphatidylethanolamine, PS - Phosphatidylserine, PC Phosphatidylcholine and PI3P - PtdIns (3)P (adapted from Dumas et al., 2001).

Towards this end, we first validated the ZFYVE27 self-association properties, furthermore, narrowed down the core interaction region to the third putative transmembrane (TM)/hydrophobic region (HR3) of the protein. Surprisingly, the identified core interaction region is dispensable for its interaction with full-length counterpart, thus highlighting the presence of other functional/structural regions through which selfassociation of ZFYVE27 could be stabilized, mainly via $\mathrm{N}$-terminus region of the protein. Further investigations suggested that ZFYVE27 mainly oligomerizes into either a dimer or tetramer and also forms cytosolic dimers, probably due to covalent interaction.

Interestingly, membrane association studies revealed that ZFYVE27 might be a 
peripheral membrane protein in contrast to in silico prediction that it is an integral membrane protein, as it contains three putative TM domains. Membrane binding studies indicated that ZFYVE27 has affinity towards all the phosphorylated derivatives of Phospatidylinositol (PtdIns), but not to any other analyzed lipid molecules. ZFYVE27 being regarded as a FYVE finger protein has no conserved FYVE signature motifs like WXXD, RVC and R(R/K)HHCR motifs (Stenmark et al., 1996), distinguishing it from other FYVE proteins, suggesting it might belong to a novel FYVE sub-family. Collectively, through our observations, we speculate that ZFYVE27 might contain novel domain/motifs, which can facilitate its recruitment to both secretory as well as endocytic pathways, thus it may act as a 'master molecule' in the process of promoting neurite extension in the neurons.

Spastin has been shown to interact with ZFYVE27 through its N-terminal part, which contains a putative TM and microtubule interacting and trafficking (MIT) domains (Mannan et al., 2006a). Since, the MIT domain of spastin has been implicated in interaction with microtubules, it is interesting to address the role of TM domain in membrane localization of spastin. Our membrane association studies on full-length spastin revealed that spastin is also a peripheral membrane protein suggesting that the TM domain/hydrophobic region of spastin might mediate its interaction with ZFYVE27.

\subsection{Generation of a knock-out mouse model for Zfyve27}

To evaluate the role of ZFYVE27 in vivo, a loss of function mouse model was generated. Towards this end, firstly, a Zfyve 27 knock-out targeting construct was generated using BAC (Bacterial Artificial Chromosome) mediated homologous recombination method (reviewed by Copeland et al., 2001). In brief, a BAC clone (RPCIB731A01447Q) containing the complete Zfyve27 gene (from C57B1/6 strain) was used for the generation of knock-out construct as outlined in figure 4.3. In the first step, exon 5 till exon 12 of Zfyve27, which spans the FYVE domain at the protein level, was replaced with Neomycin/Kanamycin resistant $\left(\mathrm{Neo} / \mathrm{Kan}^{\mathrm{r}}\right)$ cassette, which was PCR amplified with a primer pair consisting of $50 \mathrm{bp}$ homologous arms from either end of the planned deleted genomic Zfyve27 region. To retrieve the genomic fragment of Zfyve27 containing $\mathrm{Neo} / \mathrm{Kan}^{\mathrm{r}}$ cassette together with $5^{\prime}$, and 3' homologous wings (about 5 to $6 \mathrm{~kb}$ ) into the targeting pL253 vector, two mini arms (5' and 3') were PCR amplified (about 500 bp) and 
cloned bi-directionally into pL253 vector. In the next step, the pL253 vector with mini arms was used for BAC mediated homologous recombination to retrieve the genomic fragment of Zfyve27 containing $\mathrm{Neo} / \mathrm{Kan}^{\mathrm{r}}$ cassette. The generated final knock-out targeting construct was verified extensively with restriction digestion analysis, PCR amplification and sequencing.

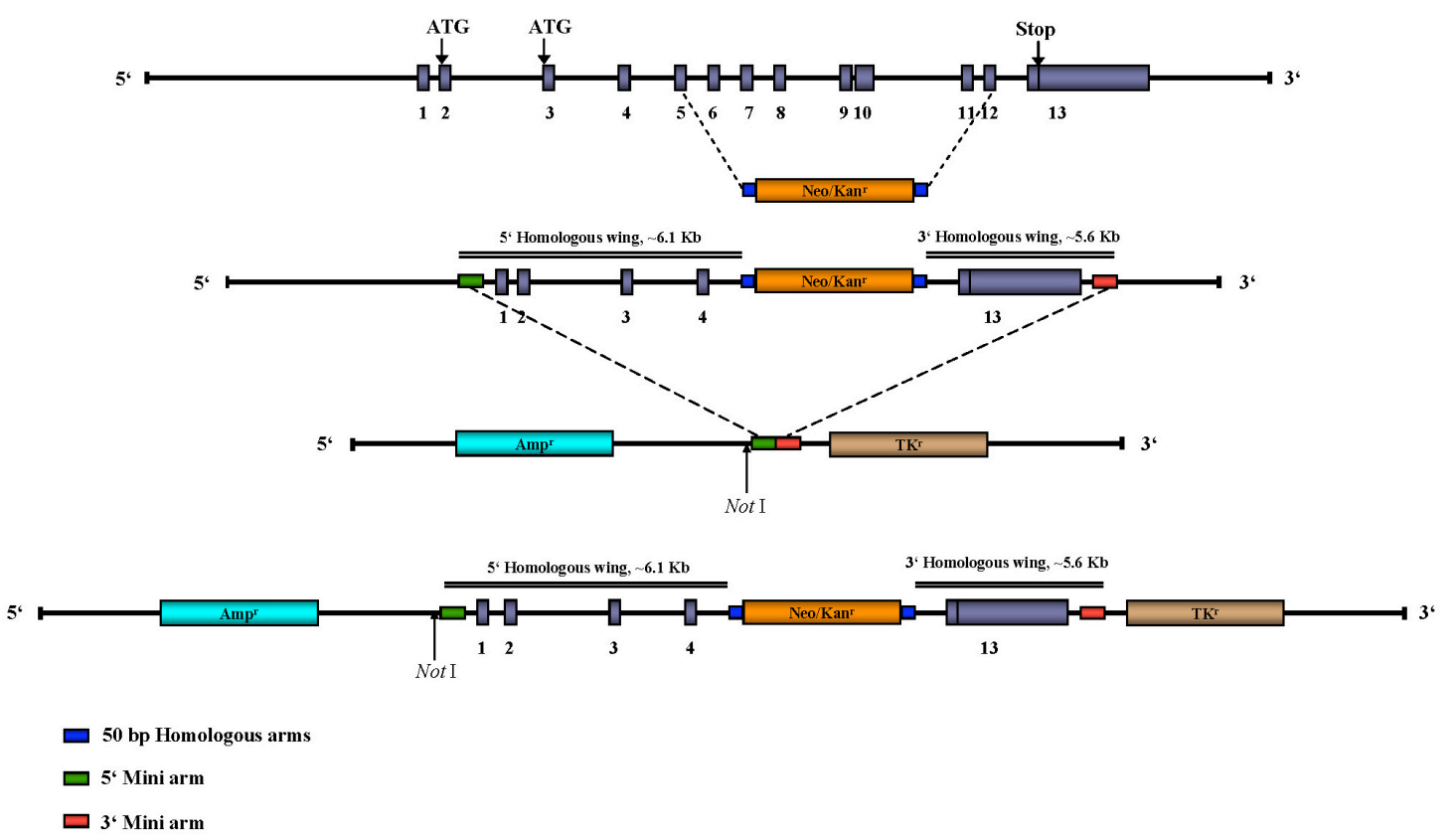

Figure 4.3. Schematic diagram depicting the generation of Zfyve 27 knock-out targeting construct. In the Zfyve27 gene a genomic fragment spanning exon 5 till exon 12 of the gene was deleted and replaced with $\mathrm{Kan} / \mathrm{Neo}^{\mathrm{r}}$ cassette (flanked with $50 \mathrm{bp}$ homologous arms as indicated) using BAC mediated homologous recombination. The resulting construct was retrieved along with 5 ' homologous wing $(\sim 6.1 \mathrm{~kb})$ and 3' homologous wing $(\sim 5.6 \mathrm{~kb})$ into the targeting vector (pL253) with the aid of homologous recombination through 5' and 3' mini arms, to obtain the final knock-out targeting construct.

The Not I linearized final knock-out targeting construct was then electroporated into mouse C57B1/6 ES (embryonic stem) cells (TS3 cell line). The resulting ES clones were screened for the homologous recombination event in Zfyve 27 gene locus using Southern blot analysis with both 5' and 3' external probes (Fig. 4.4) and 9 positive clones out of 60 screened ES clones were obtained. Next, one of the targeted ES cell clone was used for microinjection into $\mathrm{C} 57 \mathrm{Bl} / 6$-albino $\left(\mathrm{C} 57 \mathrm{Bl} / 6 \mathrm{~J}-\mathrm{Tyr}^{c-2 J} / \mathrm{J}\right) \quad\left(\mathrm{JAX}^{\circledR}\right.$ NOTES, Fall 2003) blastocysts to generate the chimeric mice. The use of C57B1/6-albino blastocysts for C57Bl/6 ES cells allowed us to distinguish the chimeric mice by coat color. We obtained 
only one chimeric mouse, which was mated with $\mathrm{C} 57 \mathrm{Bl} / 6$ mice to produce heterozygous mice. The heterozygous progenies were genotyped by PCR and also confirmed by Southern blotting. Currently, the generation of homozygous knock-out mice for Zfyve27 is in progress. Conceivably, the phenotype of knock-out mice for Zfyve 27 might mimic the pathological features of HSP.

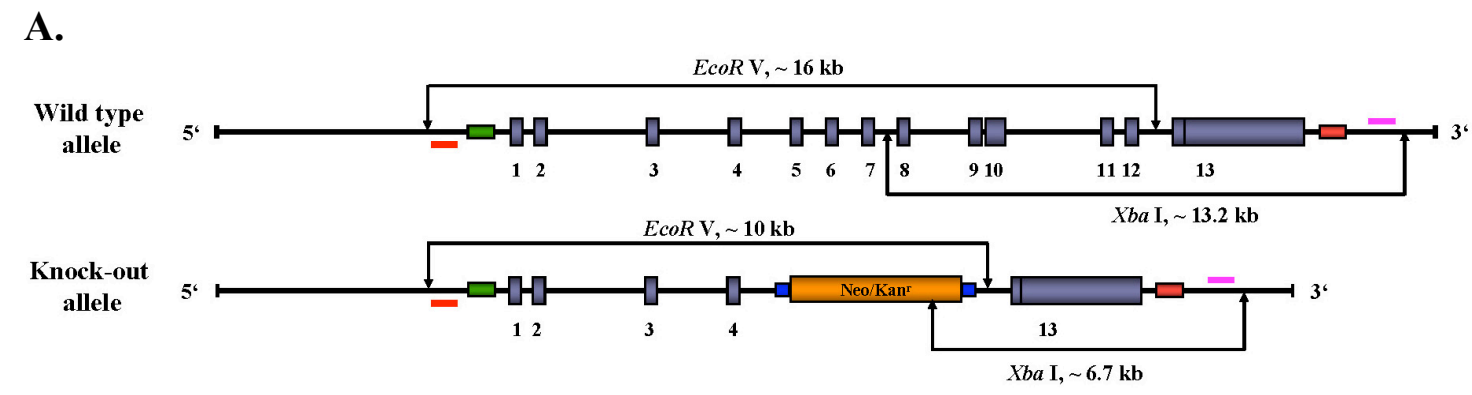

B.

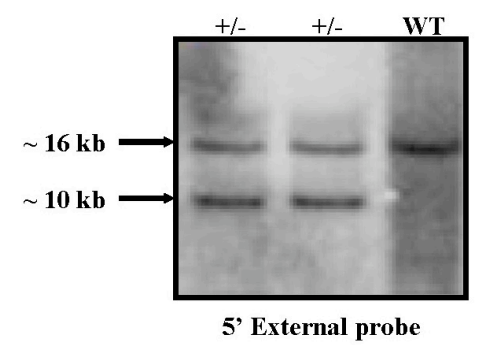

C.

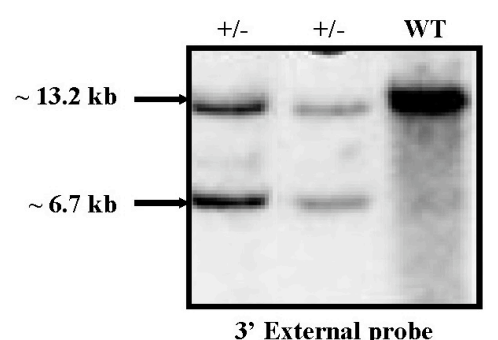

3' External probe

Figure 4.4. Schematic representation of the genomic locus for Zfyve27 wild type and knock-out allele. (A) Wild type (WT) and knock-out (KO) allele genomic locus for Zfyve27 highlighting the location of 5, external probe (red) and the expected fragments of $E c o R \mathrm{~V}$ digestion, also the 3' external probe (magenta) and the expected fragments of $X b a$ I digestion. (B) Genomic Southern blot analysis of two recombinant ES clones with 5' external probe and (C) with 3' external probe.

\subsection{Generation of conditional transgenic mouse models for ZFYVE27}

The observation that the overexpression of ZFYVE27 in terminally differentiated cells such as fibroblasts promoting neurite like structures prompted us to evaluate the possible role of ZFYVE27 in committing a neural stem cell into neuronal cell type rather than a non-neuronal cell (glia). The first indication for the role of ZFYVE27 in neuronal differentiation came from our observation that the overexpression of ZFYVE27 alone in ES cells could lead to differentiation of these cells into neuronal cells. These preliminary studies were suggestive of a possible role of ZFYVE27 in commitment of neuronal stem cells into different/specific neuronal cell types. 
In order to evaluate the in vivo significance of proposed role of ZFYVE27 in neuritogenesis, we decided to generate two conditional transgenic mouse models expressing ZFYVE27 using Cre/loxP system. In the first transgenic construct, we used a well characterized neural stem cells specific promoter from Hesl gene (Ohtsuka et al., 2006). Previously, Hes1 expression was shown to be specific to the neural stem cells in the subventricular zone of developing mice using Hes1-GFP transgenic mice (Ohtsuka et al., 2006). The second transgenic construct was generated using a strong and ubiquitous promoter, CAG [a composite promoter of the human cytomegalovirus (CMV) immediateearly enhancer and a modified chicken $\beta$-actin promoter and first intron] that was proven to be more efficient than CMV promoter in transgenic mouse models (Halbert et al., 2007).

The strategy for generation of both conditional transgenic constructs is illustrated in figure 4.5. Briefly, a pDsRed-Monomer-N1 vector expressing monomeric and soluble DsRed variant was used as a backbone and open reading frame (ORF) of human ZFYVE27 (hZFYVE27) was cloned in-frame to the upstream of DsRed. The resulting construct was then used to clone loxp-EGFP-Poly A-loxP (floxed EGFP-stop) cassette, which was obtained from pL452-EGFP vector (generated in this study). In the next step, the CMV promoter of the resulting pDsRed-floxed EGFP-stop-hZFYVE27 was replaced with either $\sim 2.1 \mathrm{~kb}$ promoter region of Hesl gene to obtain pHes1-floxed EGFP-stop-hZFYVE27DsRed transgenic construct or with $\sim 1.2 \mathrm{~kb}$ CAG promoter to obtain pCAG-floxed EGFPstop-hZFYVE27-DsRed transgenic construct (Fig. 4.5). 


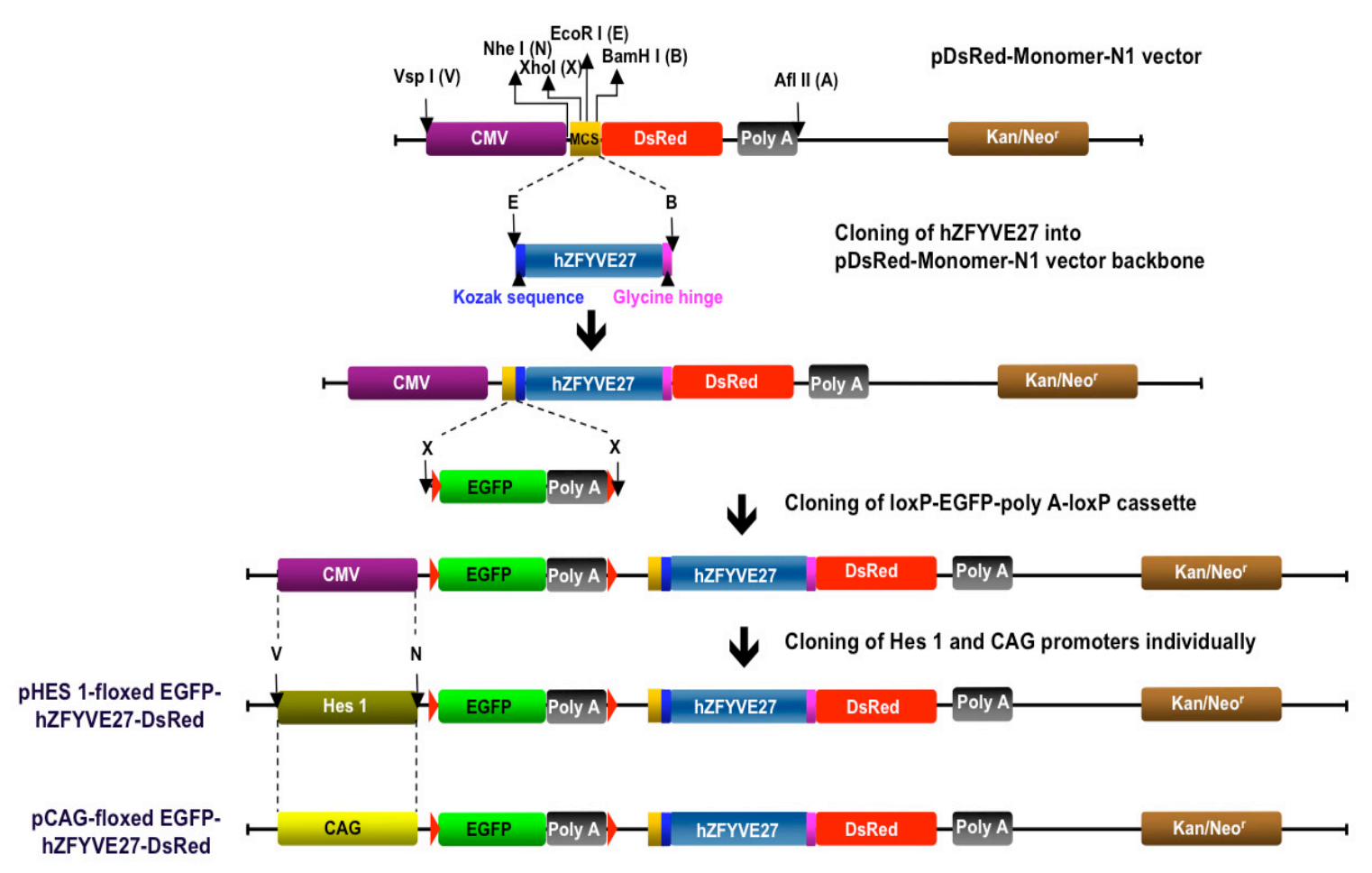

Figure 4.5. Schematic illustration of conditional transgenic constructs. Human ZFYVE7 (hZFYVE7) was cloned upstream of DsRed cassette as indicated, and in the next step floxed EGFP-poly A was cloned upstream of the hZFYVE27. The CMV promoter of the resulting construct was excised and either Hes1 promoter or CAG promoter was cloned into the same restriction sites to obtain pHes1-floxed EGFP-stophZFYVE27-DsRed transgenic construct or pCAG-floxed EGFP-stop-hZFYVE27-DsRed transgenic construct, respectively.

The final constructs were digested with $V s p$ I and $A f l$ II to retrieve the fragments containing promoter-floxed EGFP-stop- hZFYVE27-DsRed and were used for in vivo electroporation into repopulating undifferentiated spermatogonial stem cells as described previously (Dhup and Majumdhar, 2008). These putative transgenic male founders were bred with wild type FVB females to establish the transgenic mouse lines. Upon successful establishment of Hes1 and CAG transgenic mouse lines, these mice will be further bred with transgenic mice that have the Cre gene expressing only in neural stem cells or in any tissue/cell of interest. The resulting transgenic mouse progeny with Hes1/Cre transgenes or $\mathrm{CAG} / \mathrm{Cre}$ transgenes would result in the excision of EGFP-stop cassette and the activation of ZFYVE27 expression in neural stem cells or particular tissue/cell as outlined in figure 4.6. The cells (neuronal) in the transgenic mice can be tracked for the ectopic expression of ZFYVE27 by the endogenous fluorescence of DsRed. Further, isolation and 
characterization of these cells would improve our knowledge about ZFYVE27 function in neuritogenesis and might also enable us to devise a cell replacement therapy for neurodegeneration related to HSP.

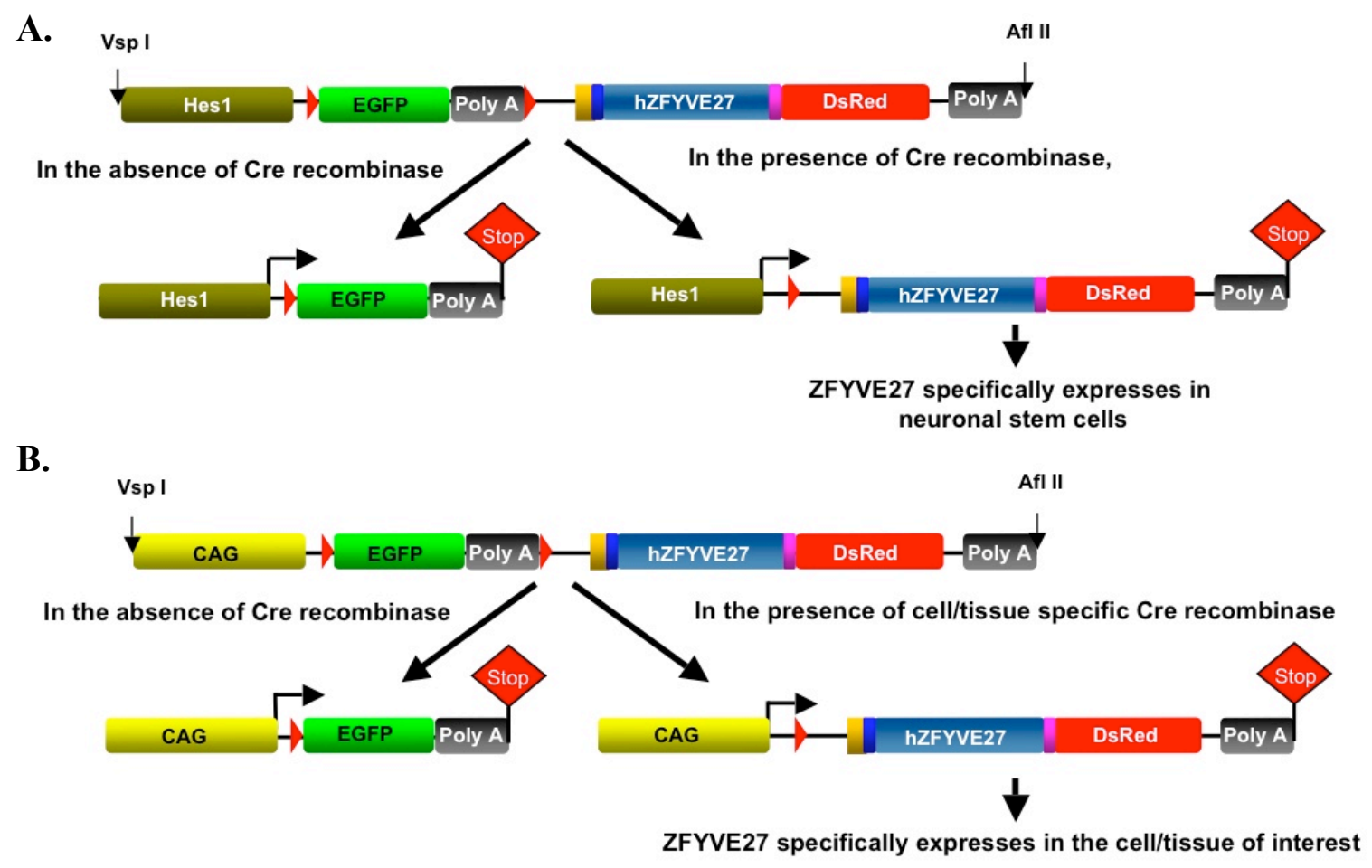

Figure 4.6. Schematic outline showing conditional activation of transgenes. (A) In the absence of Cre recombinase, the Hes1 transgenic mice would express only EGFP in neural stem cells, but when these mice are bred with another transgenic mice expressing Cre recombinase, the progeny would express ZFYVE27 specifically in neural stem cells. (B) Similarly, in the absence of Cre recombinase, the CAG transgenic mice would express only EGFP ubiquitously, but when these mice are bred with another transgenic mice carrying Cre recombinase under the control of tissue/cell specific promoter of interest, the progeny would express ZFYVE27 and will be restricted to that particular tissue/cell type. 


\subsection{Future endeavors and perspectives}

From the current study, functional and structural insights at molecular level were obtained for two closely related HSP proteins, spastin and ZFYVE27. Our structural/functional classification of SPAST-HSP mutations clearly indicates that most of the mutations may contribute to the disease by hampering either the enzyme activity or by destabilizing the monomer-monomer interactions. However, further in vivo and in vitro studies are necessary to validate these predictions, which might provide mechanistic insights into the function of spastin in MT severing and vesicular transport. These studies might also open avenues to devise therapeutic intervention for treatment of HSP.

There is an ongoing debate over the role of ZFYVE27 in causing the HSP disease. The reported ZFYVE27 mutation (a base change leading to pG191V at protein level) has recently been reported as a polymorphism in certain ethnic control population. The current studies on ZFYVE27 were aimed at understanding the significance of ZFYVE27 in HSP disease by generating the 'loss of function' mouse model for Zfyve27. Conceivably, the phenotype and the detailed characterization of this 'loss of function' mouse model would shed light on the role and relevance of ZFYVE27 in HSP. The characterization of 'gain of function' transgenic mouse models for ZFYVE27 can provide important insights about ZFYVE27 role in neuritogenesis and possibly assist in devising novel cell replacement therapy for neurodegenerative diseases, especially for HSP. 


\section{References}

Beetz C., Brodhun M., Moutzouris K., Kiehntopf M., Berndt A., Lehnert D., Deufel T., Bastmeyer M. and Schickel J. (2004) Identification of nuclear localisation sequences in spastin (SPG4) using a novel Tetra-GFP reporter system. Biochem Biophys Res Commun 318, 1079-1084.

Beetz C., Nygren A. O., Schickel J., Auer-Grumbach M., Burk K., Heide G., Kassubek J., Klimpe S., Klopstock T., Kreuz F., Otto S., Schule R., Schols L., Sperfeld A. D., Witte O. W. and Deufel T. (2006) High frequency of partial SPAST deletions in autosomal dominant hereditary spastic paraplegia. Neurology 67, 1926-1930.

Behan W. M. and Maia M. (1974) Strumpell's familial spastic paraplegia: genetics and neuropathology. J Neurol Neurosurg Psychiatry 37, 8-20.

Beyer A. (1997) Sequence analysis of the AAA protein family. Protein Sci 6, 2043-2058.

Burd C. G. and Emr S. D. (1998) Phosphatidylinositol(3)-phosphate signaling mediated by specific binding to RING FYVE domains. Mol Cell 2, 157-162.

Callaghan J., Simonsen A., Gaullier J. M., Toh B. H. and Stenmark H. (1999) The endosome fusion regulator early-endosomal autoantigen 1 (EEA1) is a dimer. Biochem J 338 (Pt 2), 539543.

Chinnery P. F., Keers S. M., Holden M. J., Ramesh V. and Dalton A. (2004) Infantile hereditary spastic paraparesis due to codominant mutations in the spastin gene. Neurology 63, 710712.

Claudiani P., Riano E., Errico A., Andolfi G. and Rugarli E. I. (2005) Spastin subcellular localization is regulated through usage of different translation start sites and active export from the nucleus. Exp Cell Res 309, 358-369.

Copeland N. G., Jenkins N. A. and Court D. L. (2001) Recombineering: a powerful new tool for mouse functional genomics. Nat Rev Genet 2, 769-779.

Crippa F., Panzeri C., Martinuzzi A., Arnoldi A., Redaelli F., Tonelli A., Baschirotto C., Vazza G., Mostacciuolo M. L., Daga A., Orso G., Profice P., Trabacca A., D'Angelo M. G., Comi G. P., Galbiati S., Lamperti C., Bonato S., Pandolfo M., Meola G., Musumeci O., Toscano A., Trevisan C. P., Bresolin N. and Bassi M. T. (2006) Eight novel mutations in SPG4 in a large sample of patients with hereditary spastic paraplegia. Arch Neurol 63, 750-755.

Deluca G. C., Ebers G. C. and Esiri M. M. (2004) The extent of axonal loss in the long tracts in hereditary spastic paraplegia. Neuropathol Appl Neurobiol 30, 576-584. 
Depienne C., Fedirko E., Forlani S., Cazeneuve C., Ribai P., Feki I., Tallaksen C., Nguyen K., Stankoff B., Ruberg M., Stevanin G., Durr A. and Brice A. (2007) Exon deletions of SPG4 are a frequent cause of hereditary spastic paraplegia. $J$ Med Genet 44, 281-284.

Dhup S. and Majumdar S. S. (2008) Transgenesis via permanent integration of genes in repopulating spermatogonial cells in vivo. Nat Methods 5, 601-603.

Dreveny I., Kondo H., Uchiyama K., Shaw A., Zhang X. and Freemont P. S. (2004) Structural basis of the interaction between the AAA ATPase p97/VCP and its adaptor protein $\mathrm{p} 47$. Embo J 23, 1030-1039.

Dumas J. J., Merithew E., Sudharshan E., Rajamani D., Hayes S., Lawe D., Corvera S. and Lambright D. G. (2001) Multivalent endosome targeting by homodimeric EEA1. Mol Cell 8, 947-958.

Durr A., Brice A., Serdaru M., Rancurel G., Derouesne C., Lyon-Caen O., Agid Y. and Fontaine B. (1994) The phenotype of "pure" autosomal dominant spastic paraplegia. Neurology 44, 1274-1277.

Errico A., Ballabio A. and Rugarli E. I. (2002) Spastin, the protein mutated in autosomal dominant hereditary spastic paraplegia, is involved in microtubule dynamics. Hum Mol Genet 11, $153-163$.

Errico A., Claudiani P., D'Addio M. and Rugarli E. I. (2004) Spastin interacts with the centrosomal protein NA14, and is enriched in the spindle pole, the midbody and the distal axon. Hum Mol Genet 13, 2121-2132.

Evans K., Keller C., Pavur K., Glasgow K., Conn B. and Lauring B. (2006) Interaction of two hereditary spastic paraplegia gene products, spastin and atlastin, suggests a common pathway for axonal maintenance. Proc Natl Acad Sci U S A 103, 10666-10671.

Evans K. J., Gomes E. R., Reisenweber S. M., Gundersen G. G. and Lauring B. P. (2005) Linking axonal degeneration to microtubule remodeling by Spastin-mediated microtubule severing. J Cell Biol 168, 599-606.

Fink J. K. (2003) Advances in the hereditary spastic paraplegias. Exp Neurol 184 Suppl 1, S106110.

Fonknechten N., Mavel D., Byrne P., Davoine C. S., Cruaud C., Bonsch D., Samson D., Coutinho P., Hutchinson M., McMonagle P., Burgunder J. M., Tartaglione A., Heinzlef O., Feki I., Deufel T., Parfrey N., Brice A., Fontaine B., Prud'homme J. F., Weissenbach J., Durr A. and Hazan J. (2000) Spectrum of SPG4 mutations in autosomal dominant spastic paraplegia. Hum Mol Genet 9, 637-644.

Frickey T. and Lupas A. N. (2004) Phylogenetic analysis of AAA proteins. J Struct Biol 146, 2-10.

Gaullier J. M., Simonsen A., D'Arrigo A., Bremnes B., Stenmark H. and Aasland R. (1998) FYVE fingers bind PtdIns(3)P. Nature 394, 432-433. 
Halbert C. L., Lam S. L. and Miller A. D. (2007) High-efficiency promoter-dependent transduction by adeno-associated virus type 6 vectors in mouse lung. Hum Gene Ther 18, 344-354.

Harding A. E. (1981) Hereditary "pure" spastic paraplegia: a clinical and genetic study of 22 families. J Neurol Neurosurg Psychiatry 44, 871-883.

Harding A. E. (1983) Classification of the hereditary ataxias and paraplegias. Lancet 1, 1151-1155.

Harding A. E. (1993) Hereditary spastic paraplegias. Semin Neurol 13, 333-336.

Hayakawa A., Hayes S. J., Lawe D. C., Sudharshan E., Tuft R., Fogarty K., Lambright D. and Corvera S. (2004) Structural basis for endosomal targeting by FYVE domains. J Biol Chem 279, 5958-5966.

Hazan J., Fonknechten N., Mavel D., Paternotte C., Samson D., Artiguenave F., Davoine C. S., Cruaud C., Durr A., Wincker P., Brottier P., Cattolico L., Barbe V., Burgunder J. M., Prud'homme J. F., Brice A., Fontaine B., Heilig B. and Weissenbach J. (1999) Spastin, a new AAA protein, is altered in the most frequent form of autosomal dominant spastic paraplegia. Nat Genet 23, 296-303.

Hentati A., Deng H. X., Zhai H., Chen W., Yang Y., Hung W. Y., Azim A. C., Bohlega S., Tandan R., Warner C., Laing N. G., Cambi F., Mitsumoto H., Roos R. P., Boustany R. M., Ben Hamida M., Hentati F. and Siddique T. (2000) Novel mutations in spastin gene and absence of correlation with age at onset of symptoms. Neurology 55, 1388-1390.

JAX $^{\circledR}$ NOTES. (Fall 2003) Use of Albino B6 (C57BL/6J-Tyrc-2J) Mice as Blastocysts Hosts for B6-derived ES cells. 491.

Lindsey J. C., Lusher M. E., McDermott C. J., White K. D., Reid E., Rubinsztein D. C., Bashir R., Hazan J., Shaw P. J. and Bushby K. M. (2000) Mutation analysis of the spastin gene (SPG4) in patients with hereditary spastic paraparesis. J Med Genet 37, 759-765.

Mannan A. U., Boehm J., Sauter S. M., Rauber A., Byrne P. C., Neesen J. and Engel W. (2006a) Spastin, the most commonly mutated protein in hereditary spastic paraplegia interacts with Reticulon 1 an endoplasmic reticulum protein. Neurogenetics 7, 93-103.

Mannan A. U., Krawen P., Sauter S. M., Boehm J., Chronowska A., Paulus W., Neesen J. and Engel W. (2006b) ZFYVE27 (SPG33), a novel spastin-binding protein, is mutated in hereditary spastic paraplegia. Am J Hum Genet 79, 351-357.

McDermott C. J., Grierson A. J., Wood J. D., Bingley M., Wharton S. B., Bushby K. M. and Shaw P. J. (2003) Hereditary spastic paraparesis: disrupted intracellular transport associated with spastin mutation. Ann Neurol 54, 748-759.

McDermott C. J., Burness C. E., Kirby J., Cox L. E., Rao D. G., Hewamadduma C., Sharrack B., Hadjivassiliou M., Chinnery P. F., Dalton A. and Shaw P. J. (2006) Clinical features of hereditary spastic paraplegia due to spastin mutation. Neurology 67, 45-51. 
Morino C., Kato M., Yamamoto A., Mizuno E., Hayakawa A., Komada M. and Kitamura N. (2004) A role for Hrs in endosomal sorting of ligand-stimulated and unstimulated epidermal growth factor receptor. Exp Cell Res 297, 380-391.

Namekawa M., Muriel M. P., Janer A., Latouche M., Dauphin A., Debeir T., Martin E., Duyckaerts C., Prigent A., Depienne C., Sittler A., Brice A. and Ruberg M. (2007) Mutations in the SPG3A gene encoding the GTPase atlastin interfere with vesicle trafficking in the ER/Golgi interface and Golgi morphogenesis. Mol Cell Neurosci 35, 1-13.

Nielsen E., Christoforidis S., Uttenweiler-Joseph S., Miaczynska M., Dewitte F., Wilm M., Hoflack B. and Zerial M. (2000) Rabenosyn-5, a novel Rab5 effector, is complexed with hVPS45 and recruited to endosomes through a FYVE finger domain. J Cell Biol 151, 601-612.

Oertle T. and Schwab M. E. (2003) Nogo and its paRTNers. Trends Cell Biol 13, 187-194.

Ohtsuka T., Imayoshi I., Shimojo H., Nishi E., Kageyama R. and McConnell S. K. (2006) Visualization of embryonic neural stem cells using Hes promoters in transgenic mice. Mol Cell Neurosci 31, 109-122.

Pantakani D. V., Swapna L. S., Srinivasan N. and Mannan A. U. (2008a) Spastin oligomerizes into a hexamer and the mutant spastin (E442Q) redistribute the wild-type spastin into filamentous microtubule. J Neurochem 106, 613-624.

Pantakani D. V., Zechner U., Arygriou L., Pauli S., Sauter S. M. and Mannan A. U. (2008b) Compound heterozygosity in the SPG4 gene causes hereditary spastic paraplegia. Clin Genet 73, 268-272.

Patki V., Lawe D. C., Corvera S., Virbasius J. V. and Chawla A. (1998) A functional PtdIns(3)Pbinding motif. Nature 394, 433-434.

Patrono C., Scarano V., Cricchi F., Melone M. A., Chiriaco M., Napolitano A., Malandrini A., De Michele G., Petrozzi L., Giraldi C., Santoro L., Servidei S., Casali C., Filla A. and Santorelli F. M. (2005) Autosomal dominant hereditary spastic paraplegia: DHPLCbased mutation analysis of SPG4 reveals eleven novel mutations. Hum Mutat 25, 506.

Peterson M. R., Burd C. G. and Emr S. D. (1999) Vac1p coordinates Rab and phosphatidylinositol 3-kinase signaling in Vps45p-dependent vesicle docking/fusion at the endosome. Curr Biol 9, 159-162.

Pullan L., Mullapudi S., Huang Z., Baldwin P. R., Chin C., Sun W., Tsujimoto S., Kolodziej S. J., Stoops J. K., Lee J. C., Waxham M. N., Bean A. J. and Penczek P. A. (2006) The endosome-associated protein Hrs is hexameric and controls cargo sorting as a "master molecule". Structure 14, 661-671.

Reid E., Connell J., Edwards T. L., Duley S., Brown S. E. and Sanderson C. M. (2005) The hereditary spastic paraplegia protein spastin interacts with the ESCRT-III complexassociated endosomal protein CHMP1B. Hum Mol Genet 14, 19-38. 
Roll-Mecak A. and Vale R. D. (2005) The Drosophila homologue of the hereditary spastic paraplegia protein, spastin, severs and disassembles microtubules. Curr Biol 15, 650-655.

Roll-Mecak A. and Vale R. D. (2008) Structural basis of microtubule severing by the hereditary spastic paraplegia protein spastin. Nature 451, 363-367.

Rubino M., Miaczynska M., Lippe R. and Zerial M. (2000) Selective membrane recruitment of EEA1 suggests a role in directional transport of clathrin-coated vesicles to early endosomes. J Biol Chem 275, 3745-3748.

Salinas S., Proukakis C., Crosby A. and Warner T. T. (2008) Hereditary spastic paraplegia: clinical features and pathogenetic mechanisms. Lancet Neurol 7, 1127-1138.

Salinas S., Carazo-Salas R. E., Proukakis C., Cooper J. M., Weston A. E., Schiavo G. and Warner T. T. (2005) Human spastin has multiple microtubule-related functions. $J$ Neurochem 95, 1411-1420.

Sanderson C. M., Connell J. W., Edwards T. L., Bright N. A., Duley S., Thompson A., Luzio J. P. and Reid E. (2006) Spastin and atlastin, two proteins mutated in autosomal-dominant hereditary spastic paraplegia, are binding partners. Hum Mol Genet 15, 307-318.

Sasaki Y. and Sugamura K. (2001) Involvement of Hgs/Hrs in signaling for cytokine-mediated cfos induction through interaction with TAK1 and Pak1. J Biol Chem 276, 29943-29952.

Sauter S., Miterski B., Klimpe S., Bonsch D., Schols L., Visbeck A., Papke T., Hopf H. C., Engel W., Deufel T., Epplen J. T. and Neesen J. (2002) Mutation analysis of the spastin gene (SPG4) in patients in Germany with autosomal dominant hereditary spastic paraplegia. Hum Mutat 20, 127-132.

Scott A., Chung H. Y., Gonciarz-Swiatek M., Hill G. C., Whitby F. G., Gaspar J., Holton J. M., Viswanathan R., Ghaffarian S., Hill C. P. and Sundquist W. I. (2005) Structural and mechanistic studies of VPS4 proteins. Embo J 24, 3658-3669.

Shirane M. and Nakayama K. I. (2006) Protrudin induces neurite formation by directional membrane trafficking. Science 314, 818-821.

Shoukier M., Neesen J., Sauter S. M., Argyriou L., Doerwald N., Pantakani D. V. and Mannan A. U. (2009) Expansion of mutation spectrum, determination of mutation cluster regions and predictive structural classification of SPAST mutations in hereditary spastic paraplegia. Eur J Hum Genet 17, 187-194.

Solowska J. M., Morfini G., Falnikar A., Himes B. T., Brady S. T., Huang D. and Baas P. W. (2008) Quantitative and functional analyses of spastin in the nervous system: implications for hereditary spastic paraplegia. $J$ Neurosci 28, 2147-2157.

Steiner P., Kulangara K., Sarria J. C., Glauser L., Regazzi R. and Hirling H. (2004) Reticulon 1C/neuroendocrine-specific protein-C interacts with SNARE proteins. J Neurochem 89, 569-580. 
Stenmark H., Aasland R., Toh B. H. and D'Arrigo A. (1996) Endosomal localization of the autoantigen EEA1 is mediated by a zinc-binding FYVE finger. $J$ Biol Chem 271, 2404824054.

Svenson I. K., Ashley-Koch A. E., Pericak-Vance M. A. and Marchuk D. A. (2001) A second leaky splice-site mutation in the spastin gene. Am J Hum Genet 69, 1407-1409.

Svenson I. K., Kloos M. T., Gaskell P. C., Nance M. A., Garbern J. Y., Hisanaga S., Pericak-Vance M. A., Ashley-Koch A. E. and Marchuk D. A. (2004) Intragenic modifiers of hereditary spastic paraplegia due to spastin gene mutations. Neurogenetics 5, 157-164.

Tarrade A., Fassier C., Courageot S., Charvin D., Vitte J., Peris L., Thorel A., Mouisel E., Fonknechten N., Roblot N., Seilhean D., Dierich A., Hauw J. J. and Melki J. (2006) A mutation of spastin is responsible for swellings and impairment of transport in a region of axon characterized by changes in microtubule composition. Hum Mol Genet 15, 35443558 .

Trotta N., Orso G., Rossetto M. G., Daga A. and Broadie K. (2004) The hereditary spastic paraplegia gene, spastin, regulates microtubule stability to modulate synaptic structure and function. Curr Biol 14, 1135-1147.

Tsukazaki T., Chiang T. A., Davison A. F., Attisano L. and Wrana J. L. (1998) SARA, a FYVE domain protein that recruits Smad2 to the TGFbeta receptor. Cell 95, 779-791.

van de Velde H. J., Roebroek A. J., Senden N. H., Ramaekers F. C. and Van de Ven W. J. (1994) NSP-encoded reticulons, neuroendocrine proteins of a novel gene family associated with membranes of the endoplasmic reticulum. J Cell Sci 107 (Pt 9), 2403-2416.

Wang Q., Song C., Yang X. and Li C. C. (2003) D1 ring is stable and nucleotide-independent, whereas D2 ring undergoes major conformational changes during the ATPase cycle of p97-VCP. J Biol Chem 278, 32784-32793.

White S. R., Evans K. J., Lary J., Cole J. L. and Lauring B. (2007) Recognition of C-terminal amino acids in tubulin by pore loops in Spastin is important for microtubule severing. $J$ Cell Biol 176, 995-1005.

Williams R. L. and Urbe S. (2007) The emerging shape of the ESCRT machinery. Nat Rev Mol Cell Biol 8, 355-368.

Yu W., Qiang L., Solowska J. M., Karabay A., Korulu S. and Baas P. W. (2008) The microtubulesevering proteins spastin and katanin participate differently in the formation of axonal branches. Mol Biol Cell 19, 1485-1498.

Zhu P. P., Soderblom C., Tao-Cheng J. H., Stadler J. and Blackstone C. (2006) SPG3A protein atlastin-1 is enriched in growth cones and promotes axon elongation during neuronal development. Hum Mol Genet 15, 1343-1353. 
Zhu P. P., Patterson A., Lavoie B., Stadler J., Shoeb M., Patel R. and Blackstone C. (2003) Cellular localization, oligomerization, and membrane association of the hereditary spastic paraplegia 3A (SPG3A) protein atlastin. J Biol Chem 278, 49063-49071. 


\section{Abbreviations}

AAA

$\mathrm{AD}$

AR

ATP

BAC

bp

BSA

${ }^{\circ} \mathrm{C}$

cDNA

Cre

Cy3

DAPI

DMEM

DNA

DsRed

DTT

EDTA

EGFP

ES

FBS

FITC

FYVE

$\mathrm{g}$

GST

HEPES

Hes 1

HPLC

HR

$\operatorname{hr}(\mathrm{s})$

HSP
ATPase Associated with various cellular Activities

Autosomal Dominant

Autosomal recessive

Adenosintriphosphate

Bacterial Artificial Chromosome

base pair

bovine serum albumin

degree Celsius

complementary DNA

Cyclization recombination (Cre recombinase)

indocarbocyanine

Diamidino-2-phenylindole dihydrochloride

DULBECCO’s Modified Eagles Media

Deoxyribonucleic Acid

Discosoma sp. Red Fluorescent Protein

Dithiothreitol

Ethylene Diamine Tetraacetic Acid

Enhanced Green Fluorescent Protein

Embryonic Stem

Fetal Bovine Serum

Fluorescein Isothiocyanate

Fab1p, YOTB, Vac1p and EEA1

gravity

Glutathione S-Transferase

$\mathrm{N}$-(-hydroxymethyl) piperazin, N'-3-propansulfoneacid

mouse Hairy and enhancer of split 1 (Drosophila)

High Performance Liquid Chromatograpy

Hydrophobic Regions

hour(s)

Hereditary Spastic Paraplegia 


\begin{tabular}{|c|c|}
\hline IPTG & Isopropyl- $\beta$-thiogalactopyranoside \\
\hline Kan & Kanamycin \\
\hline $\mathrm{kb}$ & kilobase pairs \\
\hline $\mathrm{KO}$ & Knock-out \\
\hline LB & Luria-Bertani \\
\hline M & Molarity \\
\hline $\mathrm{mg}$ & milligram \\
\hline $\min$ & minute \\
\hline $\mathrm{ml}$ & milliliter \\
\hline$\mu l$ & microliter \\
\hline$\mu \mathrm{m}$ & micrometer \\
\hline MIT & Microtubule Interacting domain \\
\hline MLPA & Multiplex Ligation-dependent Probe Amplification \\
\hline $\mathrm{MT}$ & Microtubule \\
\hline MTBD & Microtubule Binding Domain \\
\hline Neo & Neomycin \\
\hline NES & Nuclear Export Signal \\
\hline NLS & Nuclear Localization Signal \\
\hline NCBI & National Center for Biotechnology Information \\
\hline ng & nanogram \\
\hline OD & Optimal Density \\
\hline ORF & Open Reading Frame \\
\hline PAGE & Polyacrylamide Gel Electrophoresis \\
\hline PBS & Phosphatebuffersaline \\
\hline PCR & Polymerase Chain Reaction \\
\hline PDB & Protein Data Bank \\
\hline $\mathrm{pH}$ & preponderance of Hydrogen ions \\
\hline POLYPHEN & POLYmorphism PHENotyping \\
\hline PtdIns & Phosphatidylinositol \\
\hline QUASEP & Quantification of Allele Specific Expression by Pyrosequencing \\
\hline RNA & Ribonucleic Acid \\
\hline rpm & rotations per minute \\
\hline
\end{tabular}


RT

RT-PCR

SDS

SDS-PAGE

sec

SIFT

TBE

TE

TM

Tris

$\mathrm{w} / \mathrm{v}$

$\mathrm{Y} 2 \mathrm{H}$

ZFYVE27
Room Temperature

Reverse Transcriptase-PCR

Sodium Dodecylsulfate

SDS-Polyacrylamide gel electrophoresis

second

Sorting of Intolerant From Tolerant

Tris-Borate-EDTA-electrophoresis buffer

Tris-EDTA buffer

Transmembrane domain

Trihydroxymethylaminomethane

weight/volume

Yeast-Two Hybrid

Zinc finger FYVE domain containing protein 27 


\section{Acknowledgments}

I wish to express my sincere gratitude to Prof. Dr. med. Dr. h. c. Wolfgang Engel for his excellent guidance and many insightful conversations during the development of the ideas in this thesis work. I am honored to have such a great personality as my mentor and I thank him for helpful comments, continuous support, fruitful scientific discussions/suggestions during my whole $\mathrm{PhD}$ period.

I would like to express my deep sense of gratitude and sincere thanks to my supervisor, Dr. Asharaf-ul Mannan, whose expertise, understanding and patience, added considerably to my $\mathrm{PhD}$ experience. I appreciate his vast knowledge, personal attention, keen interest, and expert guidance during the entire course of my $\mathrm{PhD}$ work and assistance in writing this thesis.

My sincere thanks to the members of the thesis committee; Prof. Dr. Sigrid HoyerFender, PD Dr. Michael Hoppert, Prof. Dr. Ralf Ficner, Prof. Dr. Ernst Wimmer and Prof. Dr. Michael Mühlenberg for taking time out from their busy schedule to serve as my examiners and for their critical reading of my thesis.

No words can truly express my deepest gratitude to Tatjana, Karthik, Ajay, Sunil and Rajesh, who helped me and shared my ups and downs and made my stay in Goettingen a wonderful memory.

I am thankful to my lab mates Tomoko, Anna, Vanessa, Juliane, Greg, Anton, Hope, Jessica, Heike, Johannes, Lenny and all my institute colleagues for their friendship, discussions, advices, help and fantastic working environment. I extend my sincere thanks to Marta for all her help.

I am thankful to all my friends Vijay, Sridhar, Sailaja, Deepa, Kifayath, Mals, Sowmya, Durga, Kiran, Madhumathi, Manjunath, Mohan, Santosh, Pooja, Chiru, Raju, Sudhakar, Santosh and Sridhar for their continuous support.

I wouldn't be here without them, so I would like to acknowledge Sarath, Gautam, Rahul, Srinath, Suman, Sadanand, Saradhi and Prof. Varshney for their great support and scientific encouragement.

Special acknowledgments and thanks to my mother, the late Buli Veeramma and all my family members for encouragement and the moral support they provided me.

Finally, I would like to acknowledge the Almighty God for his guidance and wisdom all the way. 


\section{Curriculum Vitae}

\section{Personal Details:}

Name: $\quad$ D. V. Krishna Pantakani

$\begin{array}{ll} & \text { Born on 07.07.1979 in Tadimalla (India) } \\ \text { Address: } & \text { Kreuzbergring } 46 \\ & 37075 \text { Göttingen } \\ \text { Telephone: } & \text { 0176/62160336 } \\ \text { E-mail: } & \text { Krishna.if1@gmail.com }\end{array}$

\section{Education:}

Primary School:

1984-1989

Secondary School:

1989-1991

1991-1994

Intermediate:

1994-1996

\section{Bachelor of Science:}

1996-1999

\section{Master of Science:}

1999-2001

\section{Promotion/PhD:}

since Jan 2006
M.P.P. Elementary School, Tadimalla, India.

Z.P. High School, Tadiamalla, India.

A.P.R. School, Appalarajugudem, India

Dr. B.R.A.C.S.W.R. College, Lakshminarasapuram, India.

D.N.R. Autonomous College, Bhimavaram, India.

(Andhra University, Waltair, India)

J.K.C. College, Guntur, India.

(Acharya Nagarjuna University, Guntur, India)

Institute of Human Genetics, Georg-August University, Goettingen Topic: "Functional Characterization of Hereditary Spastic Paraplegia Proteins Spastin and ZFYVE27"

(under the supervision of Prof. Dr. Dr. W. Engel)

\section{Career:}

Jan 2003- Dec 2005:

Research Assistant, Indian Institute of Science, Bangalore, India.

(under the supervision of Prof. Umesh Varshney) 


\section{List of Publications}

1. Singh P, Talawar RK, Krishna PD, Varshney U, Vijayan M. Overexpression, purification, crystallization and preliminary X-ray analysis of uracil N-glycosylase from Mycobacterium tuberculosis in complex with a proteinaceous inhibitor. Acta Crystallogr Sect F Struct Biol Cryst Commun 2006; 62: 1231-4.

2. Kaushal PS, Talawar RK, Krishna PD, Varshney U, Vijayan M. Unique features of the structure and interactions of mycobacterial uracil-DNA glycosylase: structure of a complex of the Mycobacterium tuberculosis enzyme in comparison with those from other sources. Acta Crystallogr D Biol Crystallogr 2008; 64: 551-60.

3. Gaur R, Grasso D, Datta PP, Krishna PD, Das G, Spencer A, et al. A single mammalian mitochondrial translation initiation factor functionally replaces two bacterial factors. Mol Cell 2008; 29: 180-90.

4. Pantakani DV, Swapna LS, Srinivasan N, Mannan AU. Spastin oligomerizes into a hexamer and the mutant spastin (E442Q) redistribute the wild-type spastin into filamentous microtubule. J Neurochem 2008a; 106: 613-24.

5. Pantakani DV, Zechner U, Arygriou L, Pauli S, Sauter SM, Mannan AU. Compound heterozygosity in the SPG4 gene causes hereditary spastic paraplegia. Clin Genet 2008b; 73: 268-72.

6. Shoukier M, Neesen J, Sauter SM, Argyriou L, Doerwald N, Pantakani DV, Mannan AU. Expansion of mutation spectrum, determination of mutation cluster regions and predictive structural classification of SPAST mutations in hereditary spastic paraplegia. Eur J Hum Genet 2009; 17: 187-94.

\section{List of Manuscripts in Submission Stage}

1. Klimpe S, Zibat A, Wellek B, Shoukier M, Sauter SM, Pantakani DVK, Mannan AU. Evaluating the effect of spastin splice-site mutations by means of quantitative allelespecific expression assay in hereditary spastic paraplegia.

2. Bonn F, Pantakani DVK, Shoukier M, Langer T, Mannan AU. Functional evaluation of the SPG7 sequence variants in hereditary spastic paraplegia by yeast complementation assay.

3. Pantakani DVK and Mannan AU. ZFYVE27 (Protrudin) oligomerize as dimer/tetramer and truncated ZFYVE27 $\left(\triangle \mathrm{HR} 3^{184-208}\right)$ deters the ability of the wildtype ZFYVE27 to promote neurite extension. 